\title{
GLOBAL WELL-POSEDNESS AND STABILITY OF ELECTRO-KINETIC FLOWS
}

\author{
DIETER BOTHE, ANDRÉ FISCHER AND JÜRGEN SAAL
}

\begin{abstract}
We consider a coupled system of Navier-Stokes and NernstPlanck equations, describing the evolution of the velocity and the concentration fields of dissolved constituents in an electrolyte solution. Motivated by recent applications in the field of micro- and nanofluidics, we consider the model in such generality that electrokinetic flows are included. This prohibits employing the assumption of electroneutrality of the total solution, which is a common approach in the mathematical literature in order to determine the electrical potential. Therefore we complement the system of mass and momentum balances with a Poisson equation for the electrostatic potential, with the charge density stemming from the concentrations of the ionic species. For the resulting Navier-Stokes-Nernst-Planck-Poisson system we prove the existence of unique local strong solutions in bounded domains in $\mathbb{R}^{n}$ for any $n \geq 2$ as well as the existence of unique global strong solutions and exponential convergence to uniquely determined steady states in two dimensions.
\end{abstract}

\section{Contents}

1. Introduction 2

1.1. The mathematical model 4]

1.2. Main results 9

2. Local in time well-posedness 16

3. Global well-posedness in two dimensions 28

4. Steady states 48

5. Asymptotic behaviour 53

Acknowledgement 59

References 59

Date: August 16, 2018.

2010 Mathematics Subject Classification. Primary: 76E25, 76D05, 35B25; Secondary: $35 \mathrm{~K} 51$.

Key words and phrases. Navier-Stokes-Nernst-Planck-Poisson equations, local and global well-posedness, exponential stability of steady states. 


\section{INTRODUCTION}

Electrokinetic effects have received a lot of attention from various branches of research and applications, recently in particular in the field of micro- and nanofluidics. Two well-known phenomena are electrophoresis and electro-osmotic flow (EOF). While in electrophoresis, a dispersed (colloidal) particle is dragged through a fluid under an electrical field, EOF describes the motion of an aqueous solution past a solid wall as a response to an external electrical field. The latter offers a powerful method to manipulate liquids in mini- and micro-devices, since it does not depend on mechanical tools. Indeed, employing electrical fields to pump a liquid through a (sub)micro-channel can economize this task considerably, if there are charged solutes in dispersion; see, e.g., [28, 33. EOF can be applied, for instance, for cooling systems in micro-electronics. In analytical chemistry, electrical fields serve for the separation of chemicals according to their electrophoretic characteristics (see [24]), but it also has a great potential for promoting mixing on micro-scales [8]. For further applications we refer to [41] and the references therein.

The present paper studies the mathematical model governing electroosmotic flow. From the significance of the EOF, a proper mathematical understanding is indispensable for applications. We consider a rather general situation in which a dilute viscous solution with dissolved charged species is placed in a container with solid walls, the container being situated in an electrical field. A typical concrete case is an aqueous electrolyte solution, where of course uncharged species may also be dissolved in the solute. The externally applied electrical field induces the EOF due to the presence of charged constituents in solution. On the other hand, the ions themselves generate an intrinsic electrical field as well, which in turn also affects their fluxes. Thus, even without external electrical fields, electrical effects have to be accounted for whenever charged species are involved. Concerning the solid-liquid interface, it is well-known from electro-chemistry, see e.g. [31, that electro-chemical double layers appear on the surface, thus the surface is typically charged. In a simplified view the surface can be interpreted locally as a plate capacitor. In contrast, far away from walls or other interfaces, the net charge density is essentially zero, i.e. the solution is electro-neutral inside the bulk; see, e.g., [22, 31]. But note that in nanofluidic applications as well as in transport processes in nanopores within porous media, there is no macroscopic bulk phase and, hence, electroneutrality does not even hold in a subdomain. Even the more general approach to describe the electric potential via the Poisson-Boltzmann equation may not be accurate enough, since the Boltzmann statistics does not apply in overlapping double layers; cf. [30]. 
In the mathematical literature, the electroneutrality condition has been frequently used as a simplifying condition, see e.g. [3, 7, 42, As a consequence of electroneutrality, there also is no electrical body-force acting on the mixture, such that the equations for the fluid motion decouple. There still is a non-trivial electrical field, implicitly determined by the algebraic condition of electro-neutrality which leads to a strong coupling of the mass fluxes, but still this simplification is too restrictive here, since it rules out the electrokinetic effects.

In papers of Y.S. Choi and R. Lui, see [9, 10, as well as in papers of H. Gajewski, A. Glitzky, K. Gröger and R. Hünlich, e.g. [15, 19, 20, 21], the electro-neutrality condition is not employed. Instead, in the context of reaction-diffusion systems, a more general mathematical model comprising species mass balances with fluxes according to the Nernst-Planck equation combined with a Poisson equation for the electrical potential is investigated. However, no fluid motion is taken into account and, hence, no momentum balance is accounted for. Employing suitable Lyapunov functionals, the existence of global solutions in two dimensions is proven and a careful analysis of the long-time behavior is provided. In this approach the mass flux $j_{i}$ of constituent $i$ is composed of a Fickian diffusion term and an electro-migration term due to the electrical field.

In [35, the afore mentioned system of Nernst-Planck equation and the Poisson equation is complemented by the Navier-Stokes system. Wellposedness issues are accounted for under the assumption that all diffusivities of the individual species are constant and equal. From the physical point of view, these assumptions are rather strong. In fact, the diffusivity of one species will in general depend on the full composition of the system; cf. [11. While this dependence is negligible in the dilute case considered here, the values of the diffusivities of individual constituents will still differ significantly, up to orders of magnitude. Moreover, in [35] the electrostatic potential is assumed to satisfy the homogeneous Neumann condition, which rules out boundary charges in contrast to the possible occurrence of double layers at the boundary.

Further in some way related mathematical literature is given by [5, 23]. In [5], for instance, motion of a rigid charged macro-molecule immersed in an incompressible ionized fluid is considered and local existence of a weak solution is proved. The work 23] deals with a two carriers (anions and cations) electrophoretic model in the whole space $\mathbb{R}^{n}$. There local-in-time existence of a unique strong solution in a Hilbert space setting is proved. A major point in 23] is uniformity of the existence interval with respect to the viscosity, allowing for the inviscid limit to the Euler-Nernst-Planck-Poisson system.

Apparently, no further analytical works are available concerning the system consisting of the Navier-Stokes equations for the fluid motion, the 
Nernst-Planck equation for the species concentrations and the Poisson equation for the electrostatic potential. Therefore, our approach generalizes the ones given, for instance, in [9, 10, 15, 19, 20, 21, where electro(-reaction)diffusion systems are considered, not taking into account convection induced by fluid motion or a coupling to the Navier-Stokes equations. In this context, we also want to stress the fact that our approach does not need the electro-neutrality assumption, as is assumed e.g. in [3, 7, 42], and also allows for different diffusivities for the individual species (in contrast to [35]) as well as for non-trivial charge density on the interface. Our result on local-in-time well-posedness for the full Navier-Stokes-Nernst-Planck-Poisson system in a strong $L^{p}$-setting hence is new. The methods used for the proof of global strong solutions in two space dimensions are close to the ones utilized in [10]. The results obtained in the underlying note, however, differ from the ones derived in [10] in several respects. Apart from the fact that in [10] there is no convection, an approach in Hölder spaces is presented whereas we prefer to work in an $L^{p}$-setting. Our proof of existence and uniqueness of steady states is much simpler and we are able to remove a restrictive global electro-neutrality condition imposed in [10] for uniqueness of steady states. Moreover, we prove exponential stability of steady states which is not given in [10]. The methods applied here are related to [20, where exponential stability of steady states for concentrations is derived in the absence of fluid motion. In this regard, we note that the proof of exponential convergence to a steady state simultaneously for fluid velocity and concentrations of species is new.

This article is organized as follows. First, we give a justification of the mathematical model considered in this work, the Navier-Stokes-NernstPlanck-Poisson system, by illustrating its derivation from basic principles. We then state our main results on local well-posedness in arbitrary dimension and on global well-posedness and exponential stability in two dimensions. In Section 2 we give a proof of the local-in-time well-posedness result based on maximal regularity. Utilizing an appropriate Lyapunov functional and conservation of mass for the concentrations, which gives the starting point for a bootstrap argument, we prove in Section 3 that in two dimensions the local solution extends to a global one. The content of Section 4 is the proof of existence of a unique steady state solution to the Navier-StokesNernst-Planck-Poisson system for each given distribution of initial masses. Finally, in Section 5 we prove exponential stability of this stationary solution.

1.1. The mathematical model. The basic continuum mechanical model for transport processes in (non-reactive) electrolyte solutions consists of the Navier-Stokes equations for the mixture as a whole, coupled to a set of species equations for which the mass fluxes are modeled via the NernstPlanck equation with the electrical potential given by a Poisson equation; 
see [31]. Then, depending on the concrete situation, the Poisson equations is often replaced by either the above mentioned condition of electro-neutrality or by the Poisson-Boltzmann equation in which the concentrations are related to the electrical potential by a Boltzmann statistics. Here, in order to maintain the applicability to EOF, we keep the more general Poisson equation.

But there is one hidden difficulty which forces us to briefly reconsider the derivation of the mathematical model. Indeed, the Nernst-Planck equation in the usual form, stating that the diffusive (molar) mass fluxes $j_{i}$ are given as

$$
j_{i}=-D_{i} \nabla c_{i}-m_{i} \frac{F}{R T} z_{i} c_{i} \nabla \phi,
$$

already relies on additional assumptions. In fact, the standard derivation from the more fundamental generalized Maxwell-Stefan equations ([26]) already considers the mixture to be electrically neutral. But the resulting form is also applied to the general case which obviously requires clarification. Fortunately, it turns out that the constitutive equation (1.1) is also valid without electro-neutrality if the electrolyte solution is dilute - the case we are considering here.

We consider a fluid composed of $N+1$ constituents with molar concentrations $c_{i}$, i.e. $c_{i}=\rho_{i} / M_{i}$ with $\rho_{i}$ the mass density and $M_{i}$ the molar mass, where $i=0$ refers to the solvent in which the other species $(i=1, \ldots, N)$ are dissolved. A sufficiently fundamental starting point is the system of partial mass balances together with a common mixture momentum balance, where the diffusive fluxes of species mass are determined from the system of Maxwell-Stefan equations. The latter follow as a reduced set of balances of partial momenta, simplified by neglecting diffusive waves due to differential acceleration of the constituents. The considered balances read

$$
\begin{aligned}
\partial_{t} c_{i}+\operatorname{div}\left(c_{i} u+j_{i}\right) & =0, \quad i=0, \ldots, N, \\
\partial_{t}(\rho u)+\operatorname{div}(\rho u \otimes u-S) & =-\nabla \pi+\rho b,
\end{aligned}
$$

where

$$
\rho:=\sum_{i=0}^{N} \rho_{i}, \quad \rho u:=\sum_{i=0}^{N} \rho_{i} u_{i}, \quad \rho b:=\sum_{i=0}^{N} \rho_{i} b_{i}
$$

define the total density $\rho$, the barycentric velocity $u$ and the total body force $b$, given the individual velocities $u_{i}$ and forces $b_{i}$. The molar mass flux $j_{i}$ is defined as $j_{i}:=c_{i}\left(u_{i}-u\right)$, but also has to be modeled, since individual velocities are not accounted for. In (1.3), $S$ denotes the (viscous) extra stress and $\pi$ the pressure. Note that equations (1.2) are not independent, since total mass is conserved. Indeed,

$$
\sum_{i=0}^{N} M_{i} j_{i}=0
$$


and one of the species equations in (1.2) can be replaced by the mixture continuity equation

$$
\partial_{t} \rho+\operatorname{div}(\rho u)=0 .
$$

The closure of (1.2) and (1.3), i.e. the modeling of the $j_{i}$ is achieved by the Maxwell-Stefan equations. Formulated for molar mass concentrations and under isothermal conditions they read (cf. [26])

$$
-\sum_{k=0}^{N} \frac{x_{k} j_{i}-x_{i} j_{k}}{D_{i k}}=\frac{c_{i}}{R T} \nabla \mu_{i}-\frac{y_{i}}{R T} \nabla \pi-\frac{\rho_{i}}{R T}\left(b_{i}-b\right), \quad i=0, \ldots, N .
$$

Here $x_{i}=c_{i} / c$ with $c:=\Sigma_{k=0}^{N} c_{k}$ are the molar fractions, $D_{i j}$ the MaxwellStefan diffusivities, $R$ is the universal gas constant, $T$ the absolute temperature and $y_{i}:=\rho_{i} / \rho$ the mass fractions. For extension to the non-isothermal case, see e.g. Chapter 24 in [6]. The molar-based chemical potentials $\mu_{i}$ are defined as

$$
\mu_{i}=M_{i} \frac{\partial(\rho \psi)}{\partial \rho_{i}}=\frac{\partial(\rho \psi)}{\partial c_{i}},
$$

where $\rho \psi=\rho \psi\left(T, \rho_{0}, \ldots, \rho_{N}\right)$ denotes the free energy density of the mixture. Hence $\mu_{i}$ are functions of $T$ and all $\rho_{i}$ as well, but in the literature on chemical thermodynamics and multicomponent transport, it is common to use $\left(T, \pi, x_{0}, \ldots, x_{N}\right)$ as independent variables instead. Of course not all $x_{i}$ are independent, hence $\left(T, \pi, x_{1}, \ldots, x_{N}\right)$, say, is more appropriate. If $\mu_{i}=\tilde{\mu}_{i}\left(T, \pi, x_{j}\right)$ denotes this function, then (1.5) can be written as

$$
-\sum_{k=0}^{N} \frac{x_{k} j_{i}-x_{i} j_{k}}{D_{i k}}=\frac{c_{i}}{R T} \nabla_{\pi, T} \tilde{\mu}_{i}+\frac{\phi_{i}-y_{i}}{R T} \nabla \pi-\frac{\rho_{i}}{R T}\left(b_{i}-b\right),
$$

where $\phi_{i}$ is the volume fraction of species $i$ and

$$
\nabla_{\pi, T} \tilde{\mu}_{i}:=\sum_{j} \frac{\partial \tilde{\mu}_{i}}{\partial x_{j}} \nabla x_{j}
$$

is the "gradient taken at constant $\pi$ and $T$ ". The three terms on the righthand side of (1.6) correspond to diffusional transport due to composition gradients, pressure gradients and external forces, respectively.

In case of an electrolyte solution, the individual body force densities are

$$
b_{i}=-\frac{F}{M_{i}} z_{i} \nabla \phi,
$$

where $F$ is the Faraday constant (charge of one mole of electrons), $z_{i}$ are the charge numbers and $\phi$ is the electrical potential. The latter is assumed to be determined by the electrostatic relation

$$
-\operatorname{div}(\varepsilon \nabla \phi)=F \sum_{k=0}^{N} z_{k} c_{k}
$$


i.e. dynamic effects and the influence of magnetic fields are ignored which is usually sound for the applications which have been mentioned above. In (1.8), the material parameter $\varepsilon$ denotes the electrical permittivity of the medium.

The system of equations (1.2), (1.3), (1.4), (1.6), (1.7) and (1.8) has to be complemented by an equation of state, relating $\pi$ with $\left(T, \rho_{0}, \ldots, \rho_{N}\right)$, and by a material function modeling the free energy of the mixture; alternatively, and more common, the chemical potentials need to be modeled. These are highly complicated tasks, in general.

From here on, we only consider the case of a dilute solution, for which species 0 , say, is the solvent with $x_{0} \approx 1$ and all other species are present in trace quantities, i.e. $x_{i} \ll 1$ for $i=1, \ldots, N$. This leads to several simplifications. First, for $i=1, \ldots, N$, the Maxwell-Stefan equations reduce to

$$
-\frac{1}{D_{i 0}} j_{i}=\frac{c_{i}}{R T} \nabla_{\pi, T} \tilde{\mu}_{i}+\frac{\phi_{i}-y_{i}}{R T} \nabla \pi-\frac{\rho_{i}}{R T}\left(b_{i}-b\right) .
$$

Next, the difficult request for an equation of state is resolved by assuming the mixture to maintain constant density, which is reasonable since $\rho \approx \rho_{0}$ and the latter will be almost constant at isothermal conditions. Therefore the species equation for $i=0$ is omitted, but

$$
\rho \equiv \text { const }, \quad \operatorname{div} u=0
$$

is used instead. The effect of pressure diffusion is usually negligible, since $\phi_{i} / y_{i}$ is not far from 1 and here both $\phi_{i}$ and $y_{i}$ are even close to zero. It remains to model the chemical potentials. For a dilute solution one has, 4,

$$
\tilde{\mu}_{i}=\tilde{\mu}_{i}^{0}(T, \pi)+R T \log x_{i}, \quad i=1, \ldots N .
$$

With these simplifications and with $D_{i}:=D_{i 0}$, the diffusive fluxes $j_{i}$ from (1.9) become

$$
j_{i}=-D_{i}\left(c \nabla x_{i}+\frac{F}{R T}\left(z_{i} c_{i}-\sum_{k=0}^{N} z_{k} c_{k}\right) \nabla \phi\right), \quad i=1, \ldots, N .
$$

This is close to the Nernst-Planck form, up to the term $\sum_{k=0}^{N} z_{k} c_{k}$. The latter vanishes in the electro-neutral case, but this would restrict the applicability of the model too far. Instead, we note that in the dilute case necessarily $z_{0}=0$, hence $b_{0}=0$, and then

$$
b_{i}-b=b_{i}-\sum_{k=0}^{N} y_{k} b_{k}=\left(1-y_{i}\right) b_{i}+\sum_{i \neq k=1}^{N} y_{k} b_{k} \approx b_{i}, \quad i=1, \ldots, N .
$$

Consequently, $b$ is small as compared to $b_{i}$ (for $i$ such that $z_{i} \neq 0$ ), but this does not imply $b$ to be small as compared to the other quantities in the momentum balance. In fact the electrical forces acting on dilute components can drag the whole mixture as exploited in EOF. To sum up, using the fact 
that $c \approx c_{0}$ is nearly constant, we see that in a dilute electrolyte solution the diffusive molar mass fluxes of the solutes can be modeled via the NernstPlanck constitutive equation

$$
j_{i}=-D_{i}\left(\nabla c_{i}+\frac{F}{R T} z_{i} c_{i} \nabla \phi\right), \quad i=1, \ldots, N .
$$

Note that this derivation from the Maxwell-Stefan equations automatically also yields the Nernst-Einstein relation, saying that the mobility coefficients in the electromigration term, i.e. the $m_{i}$ in (1.1) equal $D_{i} / R T$.

Concerning (1.3), the viscous stress tensor $S$ is modeled by

$$
S=\mu\left(\nabla u+(\nabla u)^{\top}\right)
$$

with constant viscosity $\mu>0$, hence $\operatorname{div} S=\mu \Delta u$ since $u$ is divergence free by (1.10). The permittivity $\varepsilon$ in equation (1.8) for the electrostatical potential is assumed to be positive and constant. Thus the full model reads

$$
\begin{aligned}
\rho\left(\partial_{t} u+(u \cdot \nabla) u\right)-\mu \Delta u+\nabla \pi & =-F \sum_{j=1}^{N} z_{j} c_{j} \nabla \phi, \\
\operatorname{div} u & =0, \\
\partial_{t} c_{i}+\operatorname{div}\left(c_{i} u-D_{i}\left(\nabla c_{i}+\frac{F}{R T} z_{i} c_{i} \nabla \phi\right)\right) & =0, \quad i=1, \ldots, N, \\
-\varepsilon \Delta \phi & =F \sum_{j=1}^{N} z_{j} c_{j},
\end{aligned}
$$

for $(t, x) \in(0, T) \times \Omega$, where $\Omega \subset \mathbb{R}^{n}, n \geq 2$, is the domain under consideration. We note that the force term on the right hand side of (1.15) is usually called Coulomb force.

At $\partial \Omega$, we impose the boundary conditions

$$
\begin{aligned}
u & =0, \\
-D_{i}\left(\partial_{\nu} c_{i}-\frac{F}{R T} z_{i} c_{i} \partial_{\nu} \phi\right) & =0, \quad i=1, \ldots, N, \\
\partial_{\nu} \phi+\tau \phi & =\xi,
\end{aligned}
$$

for $(t, x) \in(0, T) \times \partial \Omega$, where $\nu$ denotes the outer normal to $\Omega$ and $\partial_{\nu}$ denotes the derivative in direction $\nu$.

Condition (1.19) is the commonly assumed no-slip condition for the velocity field $u$ and the no-flux condition (1.20) models impermeable walls and assures the conservation of mass in $\Omega$. Relation (1.21) requires some physical explanation. By the Maxwell equations we have

$$
\varepsilon \partial_{\nu} \phi=\sigma_{s} \quad \text { on } \partial \Omega
$$

where $\sigma_{s}$ denotes the surface charge density. A typical choice for boundary conditions for the electrical potential (see e.g. [10, 35]) are given by homogeneous Neumann conditions, implicitly claiming that the surface is charge 
free. As already mentioned, this condition is not sufficient for our situation. However, finding appropriate models for the charge density on the surface is a delicate matter. The boundary condition (1.21) is the same as employed in [20]. For a physical motivation we imagine to append a layer of (dimensionless) thickness $0<\delta \ll 1$ around the volume $\Omega$ which gives us locally a plate capacitor for which the surface charge density is known to be

$$
\sigma_{s}=\frac{\varepsilon}{\delta}\left(\phi_{\delta}-\phi\right)
$$

where $\phi_{\delta}$ is the electrical potential at the outer boundary of the layer. Plugging this equality into (1.22) we obtain

$$
\partial_{\nu} \phi+\frac{1}{\delta} \phi=\frac{1}{\delta} \phi_{\delta}
$$

The parameter $\tau:=1 / \delta>0$ in (1.21) hence refers to the local capacity of the boundary. The right-hand side $\xi$ in (1.21) depends also on an external electrical potential.

The system of equations (1.15)-(1.18), (1.19)-(1.21) complemented by the initial conditions

$$
\begin{aligned}
& u(0, x)=u^{0}(x), \quad x \in \Omega, \\
& c(0, x)=c^{0}(x), \quad x \in \Omega,
\end{aligned}
$$

represents the full model, where here and in the sequel $c=\left(c_{1}, \ldots, c_{N}\right)$ always denotes the full vector of concentrations.

1.2. Main results. Before introducing our main results let us fix some notation which will be frequently used below.

Let $n \in \mathbb{N}$ and $\Omega \subset \mathbb{R}^{n}$ be an open domain with boundary $\partial \Omega$ and $1 \leq p \leq \infty$. By $L^{p}(\Omega), L^{p}(\partial \Omega), W^{m, p}(\Omega)$ we denote the usual Lebesgue and Sobolev spaces. We do not distinguish between spaces of functions and spaces of vector fields, i.e. we write also $L^{p}(\Omega)$ for $L^{p}(\Omega)^{n}$ for example. The notion $W_{0}^{m, p}(\Omega)$ describes the closure of smooth and compactly supported functions $C_{0}^{\infty}(\Omega)$ in $W^{m, p}(\Omega)$. For $s>0, p \in(1, \infty)$ we write $W_{p}^{s}(\Omega)=$ $\left(L_{p}(\Omega), W^{m, p}(\Omega)\right)_{\theta, p}$ for the Sobolev-Slobodeckii space, where $(\cdot, \cdot)_{\theta, p}$ is the real interpolation functor with exponents $\theta, p$ and $s=\theta m$. It is worth mentioning that $W_{2}^{m}(\Omega)=W^{m, 2}(\Omega)$ for $m \in \mathbb{N}$, see [40]. Let $C_{0, \sigma}^{\infty}(\Omega)$ denote the solenoidal functions with compact support in $\Omega$ and

$$
L_{\sigma}^{p}(\Omega)=\overline{C_{0, \sigma}^{\infty}(\Omega)}\|\cdot\|_{p}
$$

denotes the space of solenoidal $L^{p}$-functions. Occasionally we write $W_{0, \sigma}^{m, p}(\Omega)$ instead of $W_{0}^{m, p}(\Omega) \cap L_{\sigma}^{p}(\Omega)$. The corresponding norms are denoted by $\|\cdot\|_{p},\|\cdot\|_{p, \partial \Omega},\|\cdot\|_{W^{m, p}}$, etc. For $J=(0, T), T \in(0, \infty]$ and $X$ a Banach space we write $L^{p}(J ; X), W^{m, p}(J ; X), W_{p}^{s}(J ; X)$ for the corresponding spaces of $X$-valued functions. For $s \neq 1 / p$ let

$$
{ }_{0} W_{p}^{s}(J, X)={\overline{C_{0}^{\infty}((0, T], X)}}^{\|\cdot\|_{W_{p}^{s}}} .
$$


Note that ${ }_{0} W_{p}^{s}(J ; X)=W_{p}^{s}(J ; X)$ for $s<1 / p$ and that for $1 / p<s<1+1 / p$ this space coincides with all functions in $W_{p}^{s}(J, X)$ having vanishing time trace at zero.

Now let $\Omega \subset \mathbb{R}^{n}, n \geq 2$ be a bounded smooth domain. Concerning the model derived in Subsection 1.1 we set the parameters $\rho, \mu, F, R T$ all to 1 for technical simplicity; all the results obtained in this work remain valid in the general case where $\rho, \mu, F, R T>0$, but constant.

Remark 1. From the functional analytic approach to the Navier-Stokes equations it is sufficient to solve for the velocity field rather than both for the velocity and the pressure field, because the pressure can be recovered once the velocity is known, see e.g. [37. In this spirit, by formal application of the Helmholtz projection $P$ (see [37]) to (1.15), solving system (1.15), (1.16), (1.19), (1.23) is equivalent to solving the problem

$$
\begin{aligned}
\partial_{t} u+A_{S} u & =-P(u \cdot \nabla) u-P\left(\Sigma_{j} z_{j} c_{j} \nabla \phi\right), \quad t>0, \\
\left.u\right|_{t=0} & =u^{0},
\end{aligned}
$$

where $A_{S}=-P \Delta$ is the Stokes operator subject to homogeneous Dirichlet boundary conditions with domain

$$
\mathcal{D}\left(A_{S}\right)=W^{2, p}(\Omega) \cap W_{0}^{1, p}(\Omega) \cap L_{\sigma}^{p}(\Omega), \quad p \in(1, \infty) .
$$

Once (1.25), (1.26) is solved, the pressure can be recovered by

$$
\nabla \pi=(I-P)\left(\Delta u-(u \cdot \nabla) u-\left(\Sigma_{j} z_{j} c_{j} \nabla \phi\right)\right) .
$$

Remark 2. Consider problem (1.18), (1.21).

(a) For the Robin-Laplacian $-\Delta_{R}$ in $L^{p}(\Omega)$ with $\mathcal{D}\left(-\Delta_{R}\right)=\{v \in$ $\left.W^{2, p}(\Omega): \partial_{\nu} v+\tau v=0\right\}, \tau>0$ a constant, it is well-known that $0 \in \rho\left(-\Delta_{R}\right)$, therefore the operator $\left(-\Delta_{R}, \mathcal{B}\right) v:=\left(-\Delta v, \partial_{\nu} v+\tau v\right)$, is a bijection from $W^{m+2, p}(\Omega)$ to $W^{m, p}(\Omega) \times W_{p}^{m+1-1 / p}(\partial \Omega), m \in \mathbb{N}$ and $1<p<\infty$, see e.g. [40, Chapter 5].

(b) We only consider time-independent functions $\xi=\xi(x)$. This will prove important in various places throughout this work.

(c) Note that once $c$ is known, the potential $\phi$ is known as well.

From Remarks 1 and 2 it suffices to consider solutions $(u, c)$ rather than solutions $(u, c, \pi, \phi)$ of the following problem:

$$
\begin{aligned}
\partial_{t} u+A_{S} u+P(u \cdot \nabla) u+P\left(\Sigma_{j} z_{j} c_{j} \nabla \phi\right) & =0, \quad t>0, \\
\partial_{t} c_{i}+\operatorname{div}\left(c_{i} u-D_{i} \nabla c_{i}-D_{i} z_{i} c_{i} \nabla \phi\right) & =0, \quad t>0, \quad x \in \Omega \\
-\varepsilon \Delta \phi & =\Sigma_{j} z_{j} c_{j}, \quad t>0, \quad x \in \Omega \\
\partial_{\nu} c_{i}+z_{i} c_{i} \partial_{\nu} \phi & =0, \quad t>0, \quad x \in \partial \Omega, \\
\partial_{\nu} \phi+\tau \phi & =\xi, \quad t>0, x \in \partial \Omega \\
u(0) & =u^{0}, \quad x \in \Omega
\end{aligned}
$$




$$
c_{i}(0)=c_{i}^{0}, \quad x \in \Omega
$$

for $i=1, \ldots, N$. System (1.28)-(1.34) will be called problem $(P)$.

We will frequently use the abbreviation

$$
J_{i}:=j_{i}+c_{i} u=-D_{i} \nabla c_{i}-D_{i} z_{i} c_{i} \nabla \phi+c_{i} u .
$$

With this notation and the no-slip condition for $u$, equations (1.29) and (1.31) can be written as

$$
\begin{array}{rll}
\partial_{t} c_{i}+\operatorname{div} J_{i}=0, & \text { in }(0, T) \times \Omega, & i=1, \ldots, N, \\
J_{i} \cdot \nu=0, & \text { on }(0, T) \times \partial \Omega, & i=1, \ldots, N .
\end{array}
$$

For the local well-posedness in any dimension $n \geq 2$ we apply maximal regularity. Let us define the usual spaces of maximal $L^{p}$-regularity for $1<$ $p<\infty$ by

$$
\begin{aligned}
\mathbb{E}_{T, p}^{u} & :=W^{1, p}\left(0, T ; L_{\sigma}^{p}(\Omega)\right) \cap L^{p}\left(0, T ; \mathcal{D}\left(A_{S}\right)\right), \\
\mathbb{E}_{T, p}^{c} & :=W^{1, p}\left(0, T ; L^{p}(\Omega)\right) \cap L^{p}\left(0, T ; W^{2, p}(\Omega)\right), \\
\mathbb{E}_{T, p} & :=\mathbb{E}_{T, p}^{u} \times \mathbb{E}_{T, p}^{c}, \\
\mathbb{X}_{p} & :=\left(W_{p}^{2-2 / p}(\Omega) \cap W_{0, \sigma}^{1, p}(\Omega)\right) \times W_{p}^{2-2 / p}(\Omega) .
\end{aligned}
$$

We also set

$$
\begin{aligned}
& \mathbb{E}_{T, p}^{\pi}:=L^{p}\left(0, T ; \widehat{W}^{1, p}(\Omega)\right) \\
& \mathbb{E}_{T, p}^{\phi}:=W^{1, p}\left(0, T ; W^{2, p}(\Omega)\right) \cap L^{p}\left(0, T ; W^{4, p}(\Omega)\right)
\end{aligned}
$$

for the spaces of the corresponding pressure and potential. Our main result on local-in-time strong solvability now reads as

Theorem 1.1. Let $\Omega \subset \mathbb{R}^{n}, n \geq 2$, be a bounded and smooth domain and let $(n+2) / 3<p<\infty, p \neq 3$. Let $\xi \in W_{p}^{3-1 / p}(\partial \Omega)$ and $\left(u^{0}, c^{0}\right) \in \mathbb{X}_{p}$. In the case $p>3$ let $c^{0}$ and $\xi$ satisfy the compatibility condition

$$
\partial_{\nu} c_{i}^{0}+\left.z_{i} c_{i}^{0} \partial_{\nu} \phi^{0}\right|_{\partial \Omega}=0
$$

where

$$
-\varepsilon \Delta \phi^{0}=\Sigma_{j} z_{j} c_{j}^{0}, \quad \partial_{\nu} \phi^{0}+\tau \phi^{0}=\xi .
$$

Then there is $T>0$ such that there exists a unique solution $(u, c) \in \mathbb{E}_{T, p}$ to problem $(P)$. The latter is equivalent to saying that

$$
(u, c, \pi, \phi) \in \mathbb{E}_{T, p} \times \mathbb{E}_{T, p}^{\pi} \times \mathbb{E}_{T, p}^{\phi}
$$

with $\pi$ given by (1.27) is the unique solution of system (1.15)-(1.21) and (1.23), (1.24). If $c^{0} \geq 0$ (componentwise) we have $c \geq 0$ as long as the solution exists. 
Remark 3. (a) The local-in-time solution $(u, c)$ remains unique, as long as the solution exists, see Remark 7 .

(b) Remark 2 and the fact that $\partial_{t}$ and $\Delta$ commute cause the potential $\phi$ to be contained in the following regularity class:

$$
\phi \in W^{1, p}\left(0, T ; W^{2, p}(\Omega)\right) \cap L^{p}\left(0, T ; W^{4, p}(\Omega)\right),
$$

if $\xi$ is smooth enough. This is accomplished by $\xi \in W_{p}^{3-1 / p}(\partial \Omega)$ which motivates the assumption on $\xi$ in Theorem 1.1. Note, however, that this is done for simplicity; this constraint can be weakened, while still obtaining the same results presented in this paper, see Remark 8 .

(c) We also remark that at this stage minimal boundary regularity is none of our major objectives. By the higher regularity for $\phi$ we work with, the approach presented in this note should at least work for domains $\Omega$ of class $C^{4}$. This is also more than enough to guarantee maximal regularity for the Stokes equations, see e.g. 32. A closer inspection of what is really needed for the potential $\phi$ in the proofs of our main Theorems (which is not done here) and again [32] will yield that even $C^{3}$ shall be enough.

(d) The compatibility condition (1.36) is required for $p>3$ only, because the trace in time for $t=0$ of the boundary spaces is only well-defined in this case.

(e) The main difficulty in proving Theorem 1.1 lies in the fact that (1.31) represents a nonlinear boundary condition which has to be treated in the according trace space

$$
\mathbb{F}_{T, p}^{c, \partial \Omega}=W_{p}^{1 / 2-1 / 2 p}\left(0, T ; L^{p}(\partial \Omega)\right) \cap L^{p}\left(0, T ; W_{p}^{1-1 / p}(\partial \Omega)\right) .
$$

(f) From the physical point of view it is important to note that the total mass of each species is conserved due to the no-flux condition at the boundary; by nonnegativity this implies $\left\|c_{i}(t)\right\|_{1} \equiv\left\|c_{i}^{0}\right\|_{1}$ as long as the solution exists.

Now, let $(u, c)$ denote the local strong solution for the case $p=2$ and let the total free energy function $E$ be defined by

$$
E(u, c):=\frac{1}{2}\|u\|_{2}^{2}+\sum_{i=1}^{N} \int_{\Omega} c_{i} \log c_{i} d x+\frac{\varepsilon}{2}\|\nabla \phi\|_{2}^{2}+\frac{\varepsilon \tau}{2}\|\phi\|_{2, \partial \Omega}^{2},
$$

for $(u, c) \in L_{\sigma}^{2}(\Omega) \times L^{2}(\Omega)$ with $c_{i} \geq 0$. Here $\frac{1}{2}\|u\|_{2}^{2}$ represents the kinetic energy, $\int c_{i} \log c_{i}$ refers to the chemical potential for species $i$, see e.g. [31, and the last two terms correspond to electrical energy. It turns out that the function $V$, given by

$$
V(t):=E(u(t), c(t)), \quad t \geq 0,
$$


is a Lyapunov functional for system $(P)$ and its derivative $\frac{\mathrm{d}}{\mathrm{d} t} V$, the dissipation rate, is given explicitly by

$$
\frac{\mathrm{d}}{\mathrm{d} t} V(t)=-\|\nabla u(t)\|_{2}^{2}-\sum_{i=1}^{N} \int_{\Omega} \frac{1}{D_{i} c_{i}(t)}\left|j_{i}(t)\right|^{2} d x \leq 0,
$$

where $j_{i}=-D_{i} \nabla c_{i}-D_{i} z_{i} c_{i} \nabla \phi$ is the flux of species $i$. For a suitable definition of the possibly degenerate integral we refer to Lemma 3.7.

In the two-dimensional case this is the cornerstone for the following result on global well-posedness.

Theorem 1.2. Let $\Omega \subset \mathbb{R}^{2}$ be bounded and smooth and let $\left(u^{0}, c^{0}\right) \in$ $W_{0, \sigma}^{1,2}(\Omega) \times\left(W^{1,2}(\Omega) \cap L^{\infty}(\Omega)\right)$ with $c_{i}^{0} \geq 0, i=1, \ldots, N$. Then the local strong solution $(u, c) \in \mathbb{E}_{T, 2}$ to $(P)$ (equivalently, $(u, c, \pi, \phi) \in \mathbb{E}_{T, 2} \times$ $\mathbb{E}_{T, 2}^{\pi} \times \mathbb{E}_{T, 2}^{\phi}$ to (1.15)-(1.21), (1.23), 1.24) from Theorem 1.1 extends to a unique global strong solution.

Remark 4. (a) Comparing the requirements on the initial data of Theorem 1.1 for $p=2$ and of Theorem 1.2 , taking into account $W_{2}^{1}(\Omega)=W^{1,2}(\Omega)$ we note that there is an additional $L^{\infty}$-condition on $c^{0}$. This is inserted for technical reasons. However, it is possible to ease this constraint, as explained in Remark 13 .

(b) We emphasize that for the global existence of solutions due to Theorem 1.2 no smallness or compatibility conditions on the data are required. We also would like to stress at this stage that concerning 3D not only the Navier-Stokes but also the Nernst-Planck equation seems to be difficult to handle. So, even under the assumption of small data, to the authors at the present stage it is unclear how to extend Theorem 1.2 to three space dimensions, see also Remark 11.

(c) From the fact that $V$ is a Lyapunov functional we readily confirm that the quantities $\|u\|_{2}$ and $\left\|c_{i} \log c_{i}\right\|_{1}$ are uniformly bounded in time. Having this information at hand it is possible to prove that the $L^{2}(\Omega)$-norm of the concentrations $c_{i}$ is uniformly bounded in time, as it is done in [10]. Applying further energy estimates, the norm of the strong solutions $(u, c)$ is shown to remain finite for finite time, whence the solution can be extended up to any time $T$.

In view of the long-time behavior of system $(P)$ we are interested in steady state solutions in two dimensions, i.e., in existence and uniqueness of solutions $\left(u^{\infty}, c^{\infty}\right)$ to the stationary system

$$
\begin{aligned}
A_{S} u+P((u \cdot \nabla) u)+P\left(\Sigma_{j} z_{j} c_{j} \nabla \phi\right) & =0, & & \\
\operatorname{div}\left(c_{i} u-D_{i} \nabla c_{i}-D_{i} z_{i} c_{i} \nabla \phi\right) & =0, & & x \in \Omega, \quad i=1, \ldots, N, \\
-\varepsilon \Delta \phi & =\Sigma_{j} z_{j} c_{j}, & & x \in \Omega, \\
\partial_{\nu} c_{i}+z_{i} c_{i} \partial_{\nu} \phi & =0, & & x \in \partial \Omega, \\
\partial_{\nu} \phi+\tau \phi & =\xi, & & x \in \partial \Omega, \ldots, N,
\end{aligned}
$$


subject to $\int_{\Omega} c_{i}^{\infty}(x) d x=m_{i}$ for given total masses $m_{i}>0$.

Theorem 1.3. Let $\Omega \subset \mathbb{R}^{2}$ be bounded and smooth and let numbers $m_{i}>0$, $i=1, \ldots, N$, be given.

(a) Then there is a unique solution

$$
\left(u^{\infty}, c^{\infty}\right) \in\left(W_{0, \sigma}^{1,2}(\Omega) \cap W^{2,2}(\Omega)\right) \times W^{2,2}(\Omega)
$$

to problem $\left(P_{s}\right)$ subject to $c_{i}^{\infty} \geq 0, \int_{\Omega} c_{i}^{\infty} d x=m_{i}, i=1, \ldots, N$. More precisely, we have $u^{\infty}=0$ and $c^{\infty} \in W^{2,2}(\Omega)$ is the unique nonnegative solution to

$$
\begin{array}{rlrl}
\operatorname{div}\left(-D_{i} \nabla c_{i}-D_{i} z_{i} c_{i} \nabla \phi\right) & =0, & i=1, \ldots, N \\
\left.\left(\partial_{\nu} c_{i}+z_{i} c_{i} \partial_{\nu} \phi\right)\right|_{\partial \Omega} & =0, & i=1, \ldots, N \\
-\varepsilon \Delta \phi & =\Sigma_{j} z_{j} c_{j}, & \\
\left.\left(\partial_{\nu} \phi+\tau \phi\right)\right|_{\partial \Omega} & =\xi & &
\end{array}
$$

subject to $\int_{\Omega} c_{i}^{\infty} d x=m_{i}, i=1, \ldots, N$.

The pressure $\pi^{\infty}$ in the equilibrium state is given by $\pi^{\infty}=\Sigma_{j} c_{j}^{\infty}$ up to a constant.

(b) Let $\left(u^{0}, c^{0}\right) \in W_{0, \sigma}^{1,2}(\Omega) \times\left(W^{1,2}(\Omega) \cap L^{\infty}(\Omega)\right)$ with $c_{i}^{0} \geq 0$ and $\int_{\Omega} c_{i}^{0}(x) d x=m_{i}, i=1, \ldots, N$. Let $c^{\infty} \in W^{2,2}(\Omega)$ be the unique nonnegative solution to $\left(P_{s}^{\prime}\right)$ subject to $\int_{\Omega} c_{i}^{\infty} d x=m_{i}, i=1, \ldots, N$, and let $(u, c)$ be the unique global strong solution to $(P)$ with initial data $\left(u^{0}, c^{0}\right)$ from Theorem 1.2, then

$$
\lim _{t \rightarrow \infty}(u(t), c(t))=\left(0, c^{\infty}\right)
$$

in $W_{0, \sigma}^{1,2}(\Omega) \times W^{1,2}(\Omega)$.

It is worth to mention that there are no equilibrium states with non-trivial velocity, i.e. with Theorem 1.3 the long-time dynamics of the mixture is determined by the Nernst-Planck equation and the Poisson equation, problem $\left(P_{s}^{\prime}\right)$, and the initial masses $m_{i}=\int c_{i}^{0}(x) d x>0$.

The proof of Theorem 1.3 can be outlined as follows. For existence we employ the global strong solution from Theorem 1.2. Indeed, let $0 \leq c_{i}^{0} \in$ $W^{1,2}(\Omega) \cap L^{\infty}(\Omega)$ with $\int_{\Omega} c_{i}^{0}(x) d x=m_{i}, i=1, \ldots, N$, and $u^{0} \in W_{0, \sigma}^{1,2}(\Omega)$ be arbitrary and let $(u, c)$ be the corresponding global strong solution from Theorem 1.2. The estimates derived in the proof of Theorem 1.2 below imply compactness of the semi-orbits $(u, c)$ in $L_{\sigma}^{2}(\Omega) \times L^{2}(\Omega)$, where any accumulation point turns out to be a steady state. From mass conservation we know that each constituent's mass is conserved, hence a steady state for masses $m_{i}$ is found.

With the help of an alternative representation of the dissipation rate (1.37) it is possible to obtain a characterization of steady states, which allows us to conclude the uniqueness of steady states subject to prescribed masses $m_{i}>0$. 
With this reasoning, assertion $(b)$ of Theorem 1.3 essentially follows as a by-product of $(a)$ : by compactness of semi-orbits and the uniqueness of the accumulation point, the steady state, the system converges to an equilibrium as time tends to infinity. This is first obtained with respect to the $L^{2}$ topology, but with additional effort convergence with respect to $W^{1,2}$ can be proven.

Concerning the rate of convergence, we have the following result.

Theorem 1.4. Let $\Omega \subset \mathbb{R}^{2}$ be bounded and smooth, let $\left(u^{0}, c^{0}\right) \in W_{0, \sigma}^{1,2}(\Omega) \times$ $\left(W^{1,2}(\Omega) \cap L^{\infty}(\Omega)\right)$ with $c_{i}^{0} \geq 0$ and $\int_{\Omega} c_{i}^{0} d x=m_{i}, i=1, \ldots, N$, let $c^{\infty} \in$ $W^{2,2}(\Omega)$ be the unique nonnegative solution to $\left(P_{s}^{\prime}\right)$ with $\int_{\Omega} c_{i}^{\infty}=m_{i}, i=$ $1, \ldots, N$, and let $(u, c)$ be the global strong solution to $(P)$ from Theorem 1.2 with initial data $\left(u^{0}, c^{0}\right)$. Then there are constants $C, \omega>0$ such that

$$
\|u(t)\|_{2}+\left\|c(t)-c^{\infty}\right\|_{1} \leq C e^{-\omega t}
$$

In order to derive exponential convergence results, the so-called entropy method has been widely used, c.f. [13, [20] and references therein. The idea is the following: Consider a Lyapunov functional $\Psi(\psi(t))$ with

$$
\frac{\mathrm{d}}{\mathrm{d} t} \Psi(\psi(t))=-D(\psi(t)),
$$

where $\psi$ represents the state of the (physical) system, the dissipation rate $D$ is nonnegative and $\Psi(\psi)=0$ if and only if $\psi$ is an equilibrium. Suppose that $\Psi(\psi(t)) \leq C D(\psi(t))$ holds for some constant $C>0$. Then Gronwall's inequality implies $\Psi(\psi(t)) \leq \Psi(0) \exp (-t / C)$, i.e. the value of the Lyapunov functional decreases with exponential speed. In mathematical works it seems quite common to refer to $\Psi$ as the entropy. Note, however, that this notion can be misleading, since the entropy is a physical quantity in thermodynamics which is a priori not connected with a generic Lyapunov functional.

In (5.1) we define

$$
\Psi(t):=E(u(t), c(t))-E\left(0, c^{\infty}\right)
$$

From the physical point of view $\Psi(t)$ can be interpreted as the difference of the total free energy at time $t$ and the total free energy in the equilibrium state. The main task for proving Theorem 1.4 lies in the proof of the exponential decay of $\Psi$. To this end, note that $\frac{\mathrm{d}}{\mathrm{d} t} \Psi=\frac{\mathrm{d}}{\mathrm{d} t} V$, so there is an explicit representation for the derivative. It is therefore sufficient to show $\Psi(t) \leq C \frac{\mathrm{d}}{\mathrm{d} t} \Psi(t)$ for some constant $C>0$. Performing a similar approach as in 20] this can be shown by an indirect (somewhat technical) reasoning, see Theorem 5.1. The assertion of Theorem 1.4 then follows by a straight forward calculation. 


\section{LOCAL IN TIME WELL-POSEDNESS}

Let $\Omega \subset \mathbb{R}^{n}$ be bounded with smooth boundary, $n \geq 2$. By means of maximal $L^{p}$-regularity we will show local well-posedness simultaneously for system (1.28)-(1.34) and for system (1.28)-(1.34) with (1.29) and (1.31) replaced by

$$
\begin{aligned}
\partial_{t} c_{i}+\operatorname{div}\left(-D_{i} \nabla c_{i}-D_{i} z_{i} c_{i}^{+} \nabla \phi+c_{i} u\right)=0, & t>0, x \in \Omega \\
\partial_{\nu} c_{i}+z_{i} c_{i}^{+} \partial_{\nu} \phi & =0, \quad t>0, x \in \partial \Omega,
\end{aligned}
$$

where $f^{+}(x)=\max \{0, f(x)\}$ for $f: \mathbb{R}^{n} \rightarrow \mathbb{R}$.

This auxiliary problem will be denoted by $\left(P^{\prime}\right)$. Its well-posedness will give us the possibility to derive nonnegativity of concentrations $c_{i}$ in an easy way without applying the strong maximum principle. In the case of classical solutions $(u, c)$ the maximum principle implies that concentrations $c_{i}$ are nonnegative, see e.g. [10]. The reason why we hesitate to follow this line lies in the fact that, in general, strong solutions $(u, c)$, see Definiton 2.1, are not classical. Nevertheless it is possible to show that $(u, c)$ is classical via maximal regularity and bootstrapping. Since due to the strong coupling of the differential equations of $(P)$ this is rather technical, we believe that our approach to nonnegativity, inspired by [15], is appropriate.

Definition 2.1 (Strong solution). Let $\Omega \subset \mathbb{R}^{n}, n \geq 2$, be bounded and smooth, $T>0$ and $1<p<\infty$. The function

$$
(u, c) \in W^{1, p}\left(0, T ; L_{\sigma}^{p}(\Omega) \times L^{p}(\Omega)\right) \cap L^{p}\left(0, T ; \mathcal{D}\left(A_{S}\right) \times W^{2, p}(\Omega)\right)
$$

is called strong solution to $(P)$ resp. $\left(P^{\prime}\right)$ if $(u, c)$ satisfies $(P)$ resp. $\left(P^{\prime}\right)$ almost everywhere.

Remark 5. In order to show local well-posedness for $(P)$ it is sufficient to show that $\left(P^{\prime}\right)$ is well-posed. The reason lies in the fact that the (nonlinear) terms in $\left(P^{\prime}\right)$ have less regularity as compared to those in $(P)$. To be precise, for $c \in W^{1, p}\left(0, T ; L^{p}(\Omega)\right) \cap L^{p}\left(0, T ; W^{2, p}(\Omega)\right)$ we only know that $c^{+} \in W^{1, p}\left(0, T ; L^{p}(\Omega)\right) \cap L^{p}\left(0, T ; W^{1, p}(\Omega)\right)$, cf. [18]. Hence, once we have

proved local well-posedness for $\left(P^{\prime}\right)$, the same line of arguments yields local well-posedness for the original problem $(P)$.

Following this approach we first have to examine the associated linear problem and prove maximal regularity for the corresponding solution operator. In the next step we write the full problem as a fixed-point equation and apply the contraction mapping theorem to deduce a unique fixed point, the local-in-time strong solution.

In order to sort the terms in $\left(P^{\prime}\right)$ into linear and nonlinear terms, we split the potential $\phi$ into $\phi_{1}$ and $\phi_{2}$ satisfying

$$
\begin{array}{llll}
-\varepsilon \Delta \phi_{1}=0 & \text { in } \Omega, & \partial_{\nu} \phi_{1}+\tau \phi_{1}=\xi & \text { on } \partial \Omega, \\
-\varepsilon \Delta \phi_{2}=\Sigma_{j} z_{j} c_{j} & \text { in } \Omega, & \partial_{\nu} \phi_{2}+\tau \phi_{2}=0 & \text { on } \partial \Omega .
\end{array}
$$


In view of Remark 2 the functions $\phi_{1}$ and $\phi_{2}$ are well-defined, if the corresponding right-hand sides in (2.1) and (2.2) belong to suitable regularity classes. This yields the following nonlinear problem,

$$
\begin{array}{rlrl}
\partial_{t} u+A_{S} u+P\left[\Sigma_{j} z_{j} c_{j} \nabla \phi_{1}\right] & =F(u, c), & & \\
\partial_{t} c_{i}-D_{i} \Delta c_{i} & =G_{i}(u, c), & & i=1, \ldots, N, \\
\partial_{\nu} c_{i} & =H_{i}(c), & & i=1, \ldots, N . \\
\left.u\right|_{t=0} & =u^{0}, & & \\
\left.c_{i}\right|_{t=0} & =c_{i}^{0}, & i=1, \ldots, N,
\end{array}
$$

where the nonlinear terms are defined by

$$
\begin{aligned}
F(u, c) & =-P((u \cdot \nabla) u)-P\left(\Sigma_{j} z_{j} c_{j} \nabla \phi_{2}(c)\right), \\
G_{i}(u, c)=D_{i} z_{i}\left[\nabla c_{i}^{+} \cdot\left(\nabla \phi_{1}+\nabla \phi_{2}(c)\right)-\right. & \left.c_{i}^{+} \Delta \phi_{2}(c)\right]-\nabla c_{i} \cdot u \\
& i=1, \ldots, N \\
H_{i}(c)=-z_{i}\left[c_{i}^{+} \partial_{\nu}\left(\phi_{1}+\phi_{2}(c)\right)\right], \quad & i=1, \ldots, N .
\end{aligned}
$$

The linear problem associated to (2.3)-(2.7) reads as

$$
\begin{aligned}
\partial_{t} u+A_{S} u+P\left[\Sigma_{j} z_{j} c_{j} \nabla \phi_{1}\right] & =f, \\
\partial_{t} c_{i}-D_{i} \Delta c_{i} & =g_{i}, \quad i=1, \ldots, N, \\
\partial_{\nu} c_{i} & =h_{i}, \quad i=1, \ldots, N, \\
\left.u\right|_{t=0} & =u^{0}, \\
\left.c_{i}\right|_{t=0} & =c_{i}^{0}, \quad i=1, \ldots, N,
\end{aligned}
$$

with right hand sides in suitable function spaces.

Strong $L^{p}$-solvability of this linear problem is characterized in the following lemma.

Lemma 2.2. Let $\Omega \subset \mathbb{R}^{n}, n \geq 2$, be bounded and smooth, $(n+2) / 3<p<$ $\infty, 0<T<\infty$ and $\xi \in W_{p}^{3-1 / p}(\partial \Omega)$. Then there is a unique solution

$$
\begin{aligned}
& u \in W^{1, p}\left(0, T ; L_{\sigma}^{p}(\Omega)\right) \cap L^{p}\left(0, T ; \mathcal{D}\left(A_{S}\right)\right)=: \mathbb{E}_{T, p}^{u}, \\
& c \in W^{1, p}\left(0, T ; L^{p}(\Omega)\right) \cap L^{p}\left(0, T ; W^{2, p}(\Omega)\right)=: \mathbb{E}_{T, p}^{c},
\end{aligned}
$$

to problem (2.12)-(2.16) iff the data is contained in the following regularity classes

$$
\begin{aligned}
f & \in L^{p}\left(0, T ; L_{\sigma}^{p}(\Omega)\right)=: \mathbb{F}_{T, p}^{u}, \\
g & \in L^{p}\left(0, T ; L^{p}(\Omega)\right)=: \mathbb{F}_{T, p}^{c}, \\
h & \in W_{p}^{1 / 2-1 / 2 p}\left(0, T ; L^{p}(\partial \Omega)\right) \cap L^{p}\left(0, T ; W_{p}^{1-1 / p}(\partial \Omega)\right)=: \mathbb{F}_{T, p}^{c, \partial \Omega}, \\
u^{0} & \in W_{p}^{2-2 / p}(\Omega) \cap W_{0, \sigma}^{1, p}(\Omega)=: \mathbb{X}_{p}^{u}, \\
c^{0} & \in W_{p}^{2-2 / p}(\Omega)=: \mathbb{X}_{p}^{c}
\end{aligned}
$$


and the initial data satisfies the following compatibility condition

$$
\partial_{\nu} c_{i}^{0}=\gamma_{0} h_{i}, \quad i=1, \ldots, N, \text { in the case } p>3,
$$

where $\gamma_{0}$ is the trace operator in time for $t=0$.

Proof. The existence of a unique solution $c \in \mathbb{E}_{T, p}^{c}$ to the parabolic problem (2.13), (2.14) and (2.16) iff the data fulfills (2.18), (2.19), (2.21) and (2.22) is well-known and contained e.g. in [12]. From $\xi \in W_{p}^{3-1 / p}(\partial \Omega)$ we obtain, cf. Remark 2, $\phi_{1} \in W^{4, p}(\Omega)$, hence $\nabla \phi_{1} \in W^{3, p}(\Omega)$. Note that $\phi_{1}$ is timeindependent. For equation (2.12) we compute

$$
\left\|c_{i} \nabla \phi_{1}\right\|_{L^{p}\left(L^{p}\right)} \leq\left\|c_{i}\right\|_{L^{p}\left(L^{p}\right)}\left\|\nabla \phi_{1}\right\|_{B U C(\bar{\Omega})},
$$

due to $W^{3, p}(\Omega) \hookrightarrow B U C(\bar{\Omega})$ from Sobolev's embedding theorem, [1]. So $\tilde{f}:=f-P\left(\Sigma_{i} z_{i} c_{i} \nabla \phi_{1}\right) \in L^{p}\left(0, T ; L_{\sigma}^{p}(\Omega)\right)$. By maximal regularity for the Stokes operator, see e.g. [38, 17, 32] the remaining claims follow.

Let $\mathbb{E}_{T, p}:=\mathbb{E}_{T, p}^{u} \times \mathbb{E}_{T, p}^{c}, \mathbb{F}_{T, p}:=\mathbb{F}_{T, p}^{u} \times \mathbb{F}_{T, p}^{c} \times \mathbb{F}_{T, p}^{c, \partial \Omega}$ and $\mathbb{X}_{p}:=\mathbb{X}_{p}^{u} \times$ $\mathbb{X}_{p}^{c}$. From Lemma 2.2 for every given $T>0$ there is a continuous solution operator

$$
L:\left\{\left(f, g, h, u^{0}, c^{0}\right) \in \mathbb{F}_{T, p} \times \mathbb{X}_{p}:(2.22) \text { is valid }\right\} \rightarrow \mathbb{E}_{T, p},
$$

for problem (2.12)-(2.16). Observe that for $h \in \mathbb{F}_{T, p}^{c, \partial \Omega}$ the trace at $t=0$ is well-defined if $p>3$. For $p \neq 3$ we set

$$
{ }_{0} \mathbb{F}_{T, p}^{c, \partial \Omega}:=\left({ }_{0} W_{p}^{1 / 2-1 / 2 p}\left(0, T ; L^{p}(\partial \Omega)\right) \cap L^{p}\left(0, T ; W_{p}^{1-1 / p}(\partial \Omega)\right)\right)
$$

and denote by ${ }_{0} \mathbb{F}_{T, p}$ the space

$$
{ }_{0} \mathbb{F}_{T, p}=\mathbb{F}_{T, p}^{u} \times \mathbb{F}_{T, p}^{c} \times{ }_{0} \mathbb{F}_{T, p}^{c, \partial \Omega} .
$$

Lemma 2.3. Let $n \geq 2,(n+2) / 3<p<\infty, p \neq 3$ and $0<T^{\prime} \leq T<\infty$. Then there is $C>0$ independent of $T^{\prime} \leq T$ such that

$$
\left\|L_{\mid 0 \mathbb{F}_{T^{\prime}, p} \times\{0\}^{2}}\right\|_{\mathcal{L}\left(\mathbb{F}_{T^{\prime}, p} \times\{0\}^{2}, \mathbb{E}_{T^{\prime}, p}\right)} \leq C,
$$

where $L$ is the solution operator from 2.24).

Proof. We abbreviate $L_{T}:=L_{\left.\right|_{0} \mathbb{F}_{T, p}}$. From [29, Lemma 2.5] for any Banach space $X$ there is a bounded extension operator $\mathcal{E}_{T}^{0}:{ }_{0} W_{p}^{s}\left(0, T^{\prime} ; X\right) \rightarrow$ ${ }_{0} W_{p}^{s}(0, T ; X)$, whose norm is independent of $T^{\prime} \leq T$ for all $s \in[0,2]$. So we may extend $(f, g, h) \in{ }_{0} \mathbb{F}_{T^{\prime}, p}$ to some $(\tilde{f}, \tilde{g}, \tilde{h}) \in{ }_{0} \mathbb{F}_{T, p}$ with $(\tilde{f}, \tilde{g}, \tilde{h})_{\mid\left(0, T^{\prime}\right)}=$ $(f, g, h)$. Thanks to the fact that $L_{T}(\tilde{f}, \tilde{g}, \tilde{h}, 0,0)_{\mid\left(0, T^{\prime}\right)}=L(f, g, h, 0,0)$ we deduce

$$
\begin{aligned}
\|L(f, g, h, 0,0)\|_{\mathbb{E}_{T^{\prime}, p}} & \leq\left\|L_{T}(\tilde{f}, \tilde{g}, \tilde{h}, 0,0)\right\|_{\mathbb{E}_{T, p}} \leq\left\|L_{T}\right\|\|(\tilde{f}, \tilde{g}, \tilde{h})\|_{0 \mathbb{F}_{T, p}} \\
& \leq C\left\|L_{T}\right\|\|(f, g, h)\|_{0 \mathbb{F}_{T^{\prime}, p}}
\end{aligned}
$$

for $(f, g, h) \in{ }_{0} \mathbb{F}_{T^{\prime}, p}$, where $C>0$ does not depend on $T^{\prime} \leq T$ by virtue of [29, Lemma 2.5]. 
Knowing the mapping properties of the linear solution operator we can now treat the nonlinear problem (2.3)-(2.7) involving the nonlinear terms $F, G, H$, given in (2.8)-(2.11). We let $N(u, c)=(F(u, c), G(u, c), H(c))$ in the following and write $\phi_{2}=\phi_{2}(c)$ for the solution to (2.2) to indicate the (linear) $c$-dependence of $\phi_{2}$. The fact that $F, G, H$ map into the "right" spaces when $p$ is chosen appropriately is the content of

Lemma 2.4. Let $n \geq 2,(n+2) / 3<p<\infty$ and $T \in(0, \infty)$. Then $N(u, c) \in \mathbb{F}_{T, p}$ for any $(u, c) \in \mathbb{E}_{T, p}^{u} \times \mathbb{E}_{T, p}^{c}$.

For the proof we have to estimate the individual terms of $N(u, c)$. However, these computations are contained in the proof of Lemma 2.6 and are therefore omitted here.

Let $\left(u^{0}, c^{0}\right) \in \mathbb{X}_{p}$ be fixed from now on. We will prove the existence of a unique local-in-time strong solution to (2.3)-(2.7) with $(u(0), c(0))=$ $\left(u^{0}, c^{0}\right)$. This is equivalent to the existence of a unique fixed point of the equation

$$
(u, c)=L\left(N(u, c), u^{0}, c^{0}\right) .
$$

It will be convenient to split the solution in a part with zero time trace at $t=0$ plus a remaining part taking care of the non-zero traces. If $c \in \mathbb{E}_{T, p}^{c}$ is a solution of the Nernst-Planck system, in the case $p>3$ due to Lemma 2.4 by taking trace of $H(c)$ we obtain

$$
\left.H(c)\right|_{t=0}=H\left(c^{0}\right) \in W^{1-3 / p}(\partial \Omega) .
$$

We set $h^{*}:=e^{t \Delta_{\partial \Omega}} H\left(c^{0}\right)$, where $\Delta_{\partial \Omega}$ denotes the Laplace-Beltrami operator on the smooth manifold $\partial \Omega$. By maximal regularity for $\Delta_{\partial \Omega}$, see e.g. [34, 25], then we have $h^{*} \in \mathbb{F}_{T, p}^{c, \partial \Omega}$ and $h^{*}(0)=H\left(c^{0}\right)$.

If $p<3$ we just set $h^{*}=0$. According to Lemma 2.2 we may define a function $\left(u^{*}, c^{*}\right)$ by

$$
\left(u^{*}, c^{*}\right):=L\left(0,0, h^{*}, u^{0}, c^{0}\right) .
$$

Subtracting (2.26) from (2.25) yields the new fixed point problem

$$
(\bar{u}, \bar{c})=L(\bar{N}(\bar{u}, \bar{c}), 0,0),
$$

where

$$
\begin{aligned}
\bar{N}(\bar{u}, \bar{c}) & :=(\bar{F}(\bar{u}, \bar{c}), \bar{G}(\bar{u}, \bar{c}), \bar{H}(\bar{c})) \\
& :=\left(F\left(\bar{u}+u^{*}, \bar{c}+c^{*}\right), G\left(\bar{u}+u^{*}, \bar{c}+c^{*}\right), H\left(\bar{c}+c^{*}\right)-h^{*}\right),
\end{aligned}
$$

for $(\bar{u}, \bar{c}) \in{ }_{0} \mathbb{E}_{T, p}:=\left\{(u, c) \in \mathbb{E}_{T, p}:(u(0), c(0))=0\right\}$.

The advantage of applying the fixed point argument to the zero trace space ${ }_{0} \mathbb{E}_{T}$ lies in the fact that the embedding constants for embeddings in time do not depend on $T$. This enables us to choose $T$ as small as we please without having the embedding constants blow up. 
Lemma 2.5. Let $p \in(1, \infty), I \in \mathbb{N}, s_{i} \in[0,2]$, and $X_{i}$ be Banach spaces for $i=1, \ldots, I$. Suppose there is $q \in(1, \infty)$ and a Banach space $Y$ such that there is a continuous embedding

$$
\bigcap_{i=1}^{I} W_{p}^{s_{i}}\left(0, T_{0} ; X_{i}\right) \hookrightarrow L^{q}\left(0, T_{0} ; Y\right) .
$$

Then for each $T^{\prime} \leq T_{0}$ there is a continuous embedding

$$
\bigcap_{i=1}^{I}{ }_{0} W_{p}^{s_{i}}\left(0, T^{\prime} ; X_{i}\right) \hookrightarrow L^{q}\left(0, T^{\prime} ; Y\right),
$$

whose embedding constant does not depend on $T^{\prime} \leq T_{0}$.

Proof. For $T^{\prime} \in\left(0, T_{0}\right]$ let $\mathcal{E}_{T^{\prime}}^{0}$ be the extension operator

$$
\mathcal{E}_{T^{\prime}}^{0}:{ }_{0} W_{p}^{s}\left(0, T^{\prime} ; X\right) \rightarrow{ }_{0} W_{p}^{s}\left(0, T_{0} ; X\right),
$$

$s \in[0,2]$, from [29, Lemma 2.5], whose norm does not depend on $T^{\prime}$; note that $\mathcal{E}_{T}^{0}$ in [29, Lemma 2.5] does not depend on $s \in[0,2]$, that is, for each $s \in[0,2]$ we have the same extension operator. Let $\mathcal{R}_{T^{\prime}}$ be the corresponding restriction operator

$$
\mathcal{R}_{T^{\prime}}: W_{p}^{s}\left(0, T_{0} ; Y\right) \rightarrow W_{p}^{s}\left(0, T^{\prime} ; Y\right)
$$

Then the claim follows, since the following diagram commutes:

$$
\begin{array}{ccc}
\bigcap_{i=1}^{I}{ }_{0} W_{p}^{s_{i}}\left(0, T^{\prime} ; X_{i}\right) & \stackrel{\mathcal{E}_{T^{\prime}}^{0}}{\longrightarrow} & \bigcap_{i=10}^{I}{ }_{0} W_{p}^{s_{i}}\left(0, T_{0} ; X\right) \\
\downarrow & & \downarrow \\
L^{q}\left(0, T^{\prime} ; Y\right) & \stackrel{\mathcal{R}_{T^{\prime}}}{\longleftarrow} & L^{q}\left(0, T_{0} ; Y\right),
\end{array}
$$

$T^{\prime} \leq T_{0}$

Remark 6. Note that, due to the embedding (2.29), the embedding constant for (2.30) can depend on $T_{0}$.

For the proof of Theorem 1.1 it will be necessary to show a contraction estimate for the map $L \circ \bar{N}$, cf. (2.27). This is somewhat technical due to the intricate structure of the trace space $\mathbb{F}_{T, p}^{c, \partial \Omega}$. Therefore we prove it as a separate lemma. 
Lemma 2.6. Let $n \geq 2,(n+2) / 3<p<\infty, 0<T^{\prime} \leq T<\infty$ and $R>0$. Then there are $\alpha, C>0$, independent of $T^{\prime}$ and $R$ such that

$$
\begin{aligned}
& \left\|\bar{N}\left(\bar{u}^{1}, \bar{c}^{1}\right)-\bar{N}\left(\bar{u}^{2}, \bar{c}^{2}\right)\right\|_{\mathbb{F}_{T^{\prime}, p}} \leq C\left\|\left(\bar{u}^{1}, \bar{c}^{1}\right)-\left(\bar{u}^{2}, \bar{c}^{2}\right)\right\|_{\mathbb{E}_{T^{\prime}, p}} \times \\
& {\left[\left\{R+\left\|u^{*}\right\|_{L^{3 p}\left(0, T^{\prime} ; L^{3 p}\right)}+\left\|\nabla u^{*}\right\|_{L^{3 p / 2}\left(0, T^{\prime} ; L^{3 p / 2}\right)}\right.\right.} \\
& \quad+\left\|c^{*}\right\|_{L^{3 p}\left(0, T^{\prime} ; L^{3 p}\right)}+\left\|c^{*}\right\|_{L^{3 p / 2}\left(0, T^{\prime} ; W^{1,3 p / 2}\right)} \\
& \quad+\left\|\nabla \phi_{2}\left(c^{*}\right)\right\|_{L^{3 p}\left(0, T^{\prime} ; L^{3 p}\right)}+\left\|\nabla \phi_{2}\left(c^{*}\right)\right\|_{L^{3 p / 2}\left(0, T^{\prime} ; W^{1,3 p / 2}\right)} \\
& \left.\quad+\left\|\partial_{t} c^{*}\right\|_{L^{p}\left(0, T^{\prime} ; L^{p}\right)}+\left\|\partial_{t}\left(\nabla \phi_{2}\left(c^{*}\right)\right)\right\|_{L^{p}\left(0, T^{\prime} ; W^{1, p}\right)}\right\} \\
& \quad+T^{\prime \alpha}\left\{\left\|c^{*}\right\|_{B U C\left(0, T^{\prime} ; W_{p}^{2-2 / p}\right)}+\left\|\nabla \phi_{1}\right\|_{W^{1,3 p}}\right) \\
& \left.\left.\quad+\left\|\nabla \phi_{2}\left(c^{*}\right)\right\|_{B U C\left(0, T^{\prime} ; B U C\right)}\right\}\right]
\end{aligned}
$$

for $\left(\bar{u}^{1}, \bar{c}^{1}\right),\left(\bar{u}^{2}, \bar{c}^{2}\right) \in B_{0} \mathbb{E}_{T^{\prime}, p}(0, R)$, the closed ball centered at 0 in ${ }_{0} \mathbb{E}_{T^{\prime}, p}$ with radius $R$.

Proof. Before estimating the individual terms in $\bar{N}\left(\bar{u}^{1}, \bar{c}^{1}\right)-\bar{N}\left(\bar{u}^{2}, \bar{c}^{2}\right)$ recall the following estimate which is obtained as a consequence of the mixed derivative theorem; for $\alpha, \beta \in \mathbb{N}, p \in(1, \infty)$ it holds for $s \in(0,1)$ that

$$
W^{\alpha, p}\left(0, T ; L^{p}(\Omega)\right) \cap L^{p}\left(0, T ; W^{\beta, p}(\Omega)\right) \hookrightarrow W_{p}^{\alpha s}\left(0, T ; W_{p}^{\beta(1-s)}(\Omega)\right) .
$$

A proof for the case $T=\infty$ and the whole space can be found in [36]. Applying suitable extensions relation (2.32) follows for our situation, see also 34. With (2.32) and Sobolev's embedding theorem it is straight forward to see that

$$
\begin{gathered}
W^{1, p}\left(0, T ; L^{p}\right) \cap L^{p}\left(0, T ; W^{2, p}\right) \hookrightarrow W_{p}^{s_{1}}\left(0, T ; W_{p}^{2-2 s_{1}}\right) \hookrightarrow L^{3 p}\left(0, T ; L^{3 p}\right), \\
\nabla\left(W^{1, p}\left(0, T ; L^{p}\right) \cap L^{p}\left(0, T ; W^{2, p}\right)\right) \\
\hookrightarrow W_{p}^{s_{2}}\left(0, T ; W_{p}^{1-2 s_{2}}\right) \\
\hookrightarrow L^{3 p / 2}\left(0, T ; L^{3 p / 2}\right)
\end{gathered}
$$

for $s_{1}=2 /(3 p)$ and $s_{2}=1 /(3 p)$. Of course (2.32)-(2.34) are also valid for $T$ substituted by $T^{\prime}$.

These facts represent crucial ingredients for the proof. Let us, for the sake of readability, introduce the following abbreviations:

$$
\hat{u}:=\bar{u}^{1}-\bar{u}^{2}, \quad \hat{c}:=\bar{c}^{1}-\bar{c}^{2}, \quad \hat{c}_{i}:=\bar{c}_{i}^{1}-\bar{c}_{i}^{2}
$$

and accordingly $\hat{c}^{+}$and $\hat{c}_{i}^{+}$. The individual terms in $\bar{N}\left(\bar{u}^{1}, \bar{c}^{1}\right)-\bar{N}\left(\bar{u}^{2}, \bar{c}^{2}\right)$ can therefore be written in the following form:

$$
\begin{aligned}
& \bar{F}\left(\bar{u}^{1}, \bar{c}^{1}\right)-\bar{F}\left(\bar{u}^{2}, \bar{c}^{2}\right)=P\left(\left(u^{*}+\bar{u}^{2}\right) \cdot \nabla \hat{u}+\hat{u} \cdot \nabla\left(u^{*}+\bar{u}^{1}\right)\right. \\
& \left.\quad+\sum_{j} z_{j}\left(\left(c_{j}^{*}-\bar{c}_{j}^{2}\right) \nabla \phi_{2}(\hat{c})+\hat{c}_{j} \nabla \phi_{2}\left(c^{*}+\hat{c}^{1}\right)\right)\right),
\end{aligned}
$$




$$
\begin{aligned}
& \bar{G}_{i}\left(\bar{u}^{1}, \bar{c}^{1}\right)-\bar{G}_{i}\left(\bar{u}^{2}, \bar{c}^{2}\right)=D_{i} z_{i}\left(\nabla \hat{c}_{i}^{+}\left(\nabla \phi_{1}+\nabla \phi_{2}\left(c^{*}+\bar{c}^{1}\right)\right)\right. \\
& \quad+\nabla\left(c_{i}^{*}+\bar{c}_{i}^{2,+}\right) \nabla \phi_{2}(\hat{c})+\left(c_{i}^{*}+\bar{c}_{i}^{2,+}\right) \Delta \phi_{2}(\hat{c}) \\
& \left.\quad+\hat{c}_{i}^{+} \Delta \phi_{2}\left(c^{*}+\bar{c}^{1}\right)\right)+\left(u^{*}+\bar{u}^{2}\right) \nabla \hat{c}_{i}+\hat{u} \cdot \nabla\left(c_{i}^{*}+\bar{c}_{i}^{1}\right) \\
& \bar{H}_{i}\left(\bar{c}^{1}\right)-\bar{H}_{i}\left(\bar{c}^{2}\right)=-z_{i}\left(\left(c_{i}^{*}+\bar{c}_{i}^{2,+}\right) \partial_{\nu} \phi_{2}(\hat{c})+\hat{c}_{i}^{+} \partial_{\nu}\left(\phi_{1}+\phi_{2}\left(c^{*}+\bar{c}^{1}\right)\right)\right) .
\end{aligned}
$$

Concerning the individual summands in $F$ and $G$ we observe that the term resulting from the convection term $(u \cdot \nabla) u$ has the weakest regularity properties as compared to the remaining terms in $F$ and $G$. Since $F, G$ have both to be estimated in $L^{p}\left(0, T^{\prime} ; L^{p}\right)$ it is sufficient to consider only the first two summands in (2.35); the other terms left can be treated in exactly the same way. By the continuity of $P$ and Hölder's inequality, there is a constant $C>0$ not depending on $T^{\prime}$ such that

$$
\begin{aligned}
& \left\|P\left(\left(u^{*}+\bar{u}^{2}\right) \nabla \hat{u}+\hat{u} \nabla\left(u^{*}+\bar{u}^{1}\right)\right)\right\|_{L^{p}\left(0, T^{\prime} ; L^{p}\right)} \\
& \leq C\left(\left(\left\|u^{*}\right\|_{L^{3 p}\left(0, T^{\prime} ; L^{3 p}\right)}+\left\|\bar{u}^{2}\right\|_{L^{3 p}\left(0, T^{\prime} ; L^{3 p}\right)}\right)\|\nabla \hat{u}\|_{L^{3 p / 2}\left(0, T^{\prime} ; L^{3 p / 2}\right)}\right. \\
& \left.\quad+\left(\left\|\nabla u^{*}\right\|_{L^{3 p / 2}\left(0, T^{\prime} ; L^{3 p / 2}\right)}+\left\|\nabla \bar{u}^{1}\right\|_{L^{3 p / 2}\left(0, T^{\prime} ; L^{3 p / 2}\right)}\right)\|\hat{u}\|_{L^{3 p}\left(0, T^{\prime} ; L^{3 p}\right)}\right) .
\end{aligned}
$$

From the fact that $\hat{u} \in{ }_{0} \mathbb{E}_{T^{\prime}, p}^{u}$ and relations (2.33), (2.34) Lemma 2.5 assures the existence of a constant $C^{\prime}>0$, not depending on $T^{\prime}$, such that

$$
\|\hat{u}\|_{L^{3 p}\left(0, T^{\prime} ; L^{3 p}\right)}+\|\nabla \hat{u}\|_{L^{3 p / 2}\left(0, T^{\prime} ; L^{3 p / 2}\right)} \leq C^{\prime}\|\hat{u}\|_{\mathbb{E}_{T^{\prime}, p}^{u}} .
$$

So combining (2.38) and (2.39) we deduce

$$
\begin{aligned}
& \left\|P\left(\left(u^{*}+\bar{u}^{2}\right) \nabla \hat{u}+\hat{u} \nabla\left(u^{*}+\bar{u}^{1}\right)\right)\right\|_{L^{p}\left(0, T^{\prime} ; L^{p}\right)} \\
& \quad \leq C\|\hat{u}\|_{\mathbb{E}_{T^{\prime}, p}}\left(R+\left\|u^{*}\right\|_{L^{3 p}\left(0, T^{\prime} ; L^{3 p}\right)}+\left\|\nabla u^{*}\right\|_{L^{3 p / 2}\left(0, T^{\prime} ; L^{3 p / 2}\right)}\right)
\end{aligned}
$$

For further estimation note that, Remark 2 .

$$
\left\|\phi_{2}(\bar{c})\right\|_{W^{1, p}\left(0, T^{\prime} ; W^{2, p}\right) \cap L^{p}\left(0, T^{\prime} ; W^{4, p}\right)} \leq C\|\bar{c}\|_{\mathbb{E}_{T^{\prime}, p}^{c}},
$$

for $\bar{c} \in{ }_{0} \mathbb{E}_{T^{\prime}, p}^{c}$, with some constant $C>0$ independent of $T^{\prime}$. So in performing analogous computations for the other summands of $\bar{F}$ and $\bar{G}$, taking into account $\hat{c}_{i}^{+} \leq\left|\hat{c}_{i}\right|$, we establish

$$
\begin{gathered}
\left\|(\bar{F}, \bar{G})\left(\bar{u}^{1}, \bar{c}^{1}\right)-(\bar{F}, \bar{G})\left(\bar{u}^{2}, \bar{c}^{2}\right)\right\|_{L^{p}\left(0, T^{\prime} ; L^{p}\right)} \leq C\left\|\left(\bar{u}^{1}, \bar{c}^{1}\right)-\left(\bar{u}^{2}, \bar{c}^{2}\right)\right\|_{\mathbb{E}_{T^{\prime}, p}} \times \\
\left(R+\left\|u^{*}\right\|_{L^{3 p}\left(0, T^{\prime} ; L^{3 p}\right)}+\left\|u^{*}\right\|_{L^{3 p / 2}\left(0, T^{\prime} ; W^{1,3 p / 2}\right)}+\left\|c^{*}\right\|_{L^{3 p}\left(0, T^{\prime} ; L^{3 p}\right)}\right. \\
\quad+\left\|c^{*}\right\|_{L^{3 p / 2}\left(0, T^{\prime} ; W^{1,3 p / 2}\right)}+\left\|\nabla \phi_{1}\right\|_{L^{3 p / 2}\left(0, T^{\prime} ; L^{3 p / 2}\right)} \\
+\left\|\nabla \phi_{2}\left(c^{*}\right)\right\|_{L^{3 p}\left(0, T^{\prime} ; L^{3 p}\right)}+\left\|\nabla \phi_{2}\left(c^{*}\right)\right\|_{L^{3 p / 2}\left(0, T^{\prime} ; W^{1,3 p / 2}\right)}
\end{gathered}
$$

for $\left(\bar{u}^{1}, \bar{c}^{1}\right),\left(\bar{u}^{2}, \bar{c}^{2}\right) \in B_{0 \mathbb{E}_{T^{\prime}, p}}(0, R)$.

We are left with the estimation of (2.37) in $\mathbb{F}_{T^{\prime}, p}^{c, \partial \Omega}$. For the treatment of this expression let us note that since $p>(n+2) / 3$ from (2.32), Sobolev's 
embedding theorem, and by choosing $\delta<\frac{1}{2 p}(3 p-n-2)$ such that $s=$ $1 / p+\delta \in(0,1)$ we have

$$
\nabla \phi \in W_{p}^{s}\left(0, T^{\prime} ; W_{p}^{3-2 s}(\Omega)\right) \hookrightarrow B U C\left(\left[0, T^{\prime}\right] ; B U C(\bar{\Omega})\right) .
$$

Because of the smoothness of $\partial \Omega$ the normal vector field $\nu$ is $B U C^{1}(\partial \Omega)$. From $B U C^{1}(\partial \Omega) \cdot L^{p}(\partial \Omega) \hookrightarrow L^{p}(\partial \Omega)$ and $B U C^{1}(\partial \Omega) \cdot W^{1, p}(\partial \Omega) \hookrightarrow$ $W^{1, p}(\partial \Omega)$ we see, by interpolation, [40], $B U C^{1}(\partial \Omega) \cdot W_{p}^{1-1 / p}(\partial \Omega) \hookrightarrow$ $W_{p}^{1-1 / p}(\partial \Omega)$; thus we may estimate

$$
\begin{gathered}
\left\|\bar{H}_{i}\left(\bar{c}^{1}\right)-\bar{H}_{i}\left(\bar{c}^{2}\right)\right\|_{\mathbb{F}_{T^{\prime}, p}^{c, \partial \Omega}} \leq C\left\|\left(c_{i}^{*}+\bar{c}_{i}^{2,+}\right) \nabla \phi_{2}(\hat{c})\right\|_{L^{p}\left(0, T^{\prime} ; W^{1, p}\right) \cap W_{p}^{1 / 2}\left(0, T^{\prime} ; L^{p}\right)} \\
+C\left\|\hat{c}_{i}^{+}\left(\nabla \phi_{1}+\nabla \phi_{2}\left(c^{*}+\bar{c}^{1}\right)\right)\right\|_{L^{p}\left(0, T^{\prime} ; W^{1, p}\right) \cap W_{p}^{1 / 2}\left(0, T^{\prime} ; L^{p}\right)},
\end{gathered}
$$

using the fact that $\mathbb{F}_{T^{\prime}, p}^{c, \partial \Omega}$ is the spacial trace space of $L^{p}\left(0, T^{\prime} ; W^{1, p}\right) \cap$ $W_{p}^{1 / 2}\left(0, T^{\prime} ; L^{p}\right)$, see e.g. [12]. Note that, due to $\bar{H}_{i}\left(\bar{c}^{j}\right) \in{ }_{0} \mathbb{F}_{T^{\prime}, p}^{c, \partial \Omega}$, the constant $C$ in (2.43) does not depend on $T^{\prime}$. This can be seen by a similar argument as in the proof of Lemma 2.5.

Let us consider the first term on the right hand side of (2.43). First we estimate the norm in $L^{p}\left(0, T^{\prime} ; W^{1, p}\right)$. Applying the Hölder inequality yields

$$
\begin{aligned}
& \left\|\left(c_{i}^{*}+\bar{c}_{i}^{2,+}\right) \nabla \phi_{2}(\hat{c})\right\|_{L^{p}\left(0, T^{\prime} ; W^{1, p}\right)} \\
& \leq\left(\left\|c_{i}^{*}\right\|_{L^{3 p / 2}\left(0, T^{\prime} ; W^{1,3 p / 2}\right)}+\left\|\bar{c}_{i}^{2}\right\|_{L^{3 p / 2}\left(0, T^{\prime} ; W^{1,3 p / 2}\right)}\right)\left\|\nabla \phi_{2}(\hat{c})\right\|_{L^{3 p}\left(0, T^{\prime} ; W^{1,3 p}\right)} .
\end{aligned}
$$

Next we use the fact that $\bar{c}_{i}^{1}(0)=\bar{c}_{i}^{1}(0)=0$ and hence also $\phi_{2}(\hat{c})(0)=0$, which gives us by applying (2.33), Lemma 2.5, and afterwards relation (2.40) that

$$
\begin{aligned}
& \left\|\left(c_{i}^{*}+\bar{c}_{i}^{2,+}\right) \nabla \phi_{2}(\hat{c})\right\|_{L^{p}\left(0, T^{\prime} ; W^{1, p}\right)} \\
& \leq C\left(\left\|c_{i}^{*}\right\|_{L^{3 p / 2}\left(0, T^{\prime} ; W^{1,3 p / 2}\right)}+R\right)\left\|\phi_{2}(\hat{c})\right\|_{\mathbb{E}_{T^{\prime}, p}^{c}} \\
& \leq C\left(\left\|c_{i}^{*}\right\|_{L^{3 p / 2}\left(0, T^{\prime} ; W^{1,3 p / 2}\right)}+R\right)\|\hat{c}\|_{\mathbb{E}_{T^{\prime}, p}^{c}} .
\end{aligned}
$$

Let us now consider the norm in $W_{p}^{1 / 2}\left(0, T^{\prime} ; L^{p}(\Omega)\right)$. We will not calculate directly with Sobolev-Slobodeckij-norms. Instead, since $\phi_{2}$ enjoys rather convenient regularity properties, we can afford to use the interpolation inequality, [40],

$$
\begin{aligned}
& \left\|\left(c_{i}^{*}+\bar{c}_{i}^{2,+}\right) \nabla \phi_{2}(\hat{c})\right\|_{W_{p}^{1 / 2}\left(0, T^{\prime} ; L^{p}\right)} \\
& \quad \leq C(T)\left\|\left(c_{i}^{*}+\bar{c}_{i}^{2,+}\right) \nabla \phi_{2}(\hat{c})\right\|_{L^{p}\left(0, T^{\prime} ; L^{p}\right)}^{1 / 2}\left\|\left(c_{i}^{*}+\bar{c}_{i}^{2,+}\right) \nabla \phi_{2}(\hat{c})\right\|_{W^{1, p}\left(0, T^{\prime} ; L^{p}\right)}^{1 / 2}
\end{aligned}
$$

The fact that the constant $C(T)$ in (2.46) does not depend on $T^{\prime}$ again can be seen by applying the extension operator in Lemma 2.5 to $(0, T)$ and 
the fact that $\left(c_{i}^{*}+\bar{c}_{i}^{2,+}\right) \nabla \phi_{2}(\hat{c}) \in{ }_{0} W_{p}^{1 / 2}\left(0, T^{\prime} ; L^{p}\right)$. Expanding the norm in $W^{1, p}\left(0, T^{\prime} ; L^{p}\right)$ gives

$$
\begin{aligned}
& \left\|\left(c_{i}^{*}+\bar{c}_{i}^{2,+}\right) \nabla \phi_{2}(\hat{c})\right\|_{W^{1, p}\left(0, T^{\prime} ; L^{p}\right)}=\left\|\left(c_{i}^{*}+\bar{c}_{i}^{2,+}\right) \nabla \phi_{2}(\hat{c})\right\|_{L^{p}\left(0, T^{\prime} ; L^{p}\right)} \\
& \quad+\left\|\partial_{t}\left(c_{i}^{*}+\bar{c}_{i}^{2,+}\right) \nabla \phi_{2}(\hat{c})\right\|_{L^{p}\left(0, T^{\prime} ; L^{p}\right)}+\left\|\left(c_{i}^{*}+\bar{c}_{i}^{2,+}\right) \partial_{t}\left(\nabla \phi_{2}(\hat{c})\right)\right\|_{L^{p}\left(0, T^{\prime} ; L^{p}\right)} .
\end{aligned}
$$

Note that, since $\phi_{2}(\hat{c})(0)=0$, we have by relations (2.40), (2.42), and Lemma 2.5 that

$$
\begin{aligned}
& \left\|\nabla \phi_{2}(\hat{c})\right\|_{B U C\left(0, T^{\prime} ; B U C\right)} \leq C(T)\left\|\nabla \phi_{2}(\hat{c})\right\|_{W_{p}^{s}\left(0, T^{\prime} ; W_{p}^{3-2 s}(\Omega)\right)} \\
& \quad \leq C^{\prime}(T)\left\|\phi_{2}(\hat{c})\right\|_{W^{1, p}\left(0, T^{\prime} ; W^{2, p}\right) \cap L^{p}\left(0, T^{\prime} ; W^{4, p}\right)} \leq C^{\prime \prime}(T)\|\hat{c}\|_{\mathbb{E}_{T^{\prime}, p}^{c}}
\end{aligned}
$$

This implies for the second term in (2.47) that

$$
\begin{aligned}
& \left\|\partial_{t}\left(c_{i}^{*}+\bar{c}_{i}^{2,+}\right) \nabla \phi_{2}(\hat{c})\right\|_{L^{p}\left(0, T^{\prime} ; L^{p}\right)} \\
& \quad \leq\left(\left\|\partial_{t} c_{i}^{*}\right\|_{L^{p}\left(0, T^{\prime} ; L^{p}\right)}+\left\|\partial_{t} \bar{c}_{i}^{2}\right\|_{L^{p}\left(0, T^{\prime} ; L^{p}\right)}\right)\left\|\nabla \phi_{2}(\hat{c})\right\|_{B U C\left(0, T^{\prime} ; B U C\right)} \\
& \quad \leq C(T)\left(\left\|\partial_{t} c_{i}^{*}\right\|_{L^{p}\left(0, T^{\prime} ; L^{p}\right)}+R\right)\|\hat{c}\|_{\mathbb{E}_{T^{\prime}, p}^{c}}
\end{aligned}
$$

For the third term in (2.47) we compute

$$
\begin{aligned}
\|\left(c_{i}^{*}\right. & \left.+\bar{c}_{i}^{2,+}\right) \partial_{t}\left(\nabla \phi_{2}(\hat{c})\right) \|_{L^{p}\left(0, T^{\prime} ; L^{p}\right)} \\
& \left.\leq\left(\left\|c_{i}^{*}\right\|_{B U C\left(0, T^{\prime} ; L^{q}\right)}+\left\|\bar{c}_{i}^{2}\right\|_{B U C\left(0, T^{\prime} ; L^{q}\right)}\right)\left\|\partial_{t}\left(\nabla \phi_{2}(\hat{c})\right)\right\|_{L^{p}\left(0, T^{\prime} ; L^{q^{\prime}}\right.}\right)
\end{aligned}
$$

with $1 / q+1 / q^{\prime}=1 / p$. From [2] it holds

$$
W^{1, p}\left(0, T^{\prime} ; L^{p}(\Omega)\right) \cap L^{p}\left(0, T^{\prime} ; W^{2, p}(\Omega)\right) \hookrightarrow B U C\left(0, T^{\prime} ; W_{p}^{2-2 / p}(\Omega)\right) .
$$

So the term $\left\|c_{i}^{*}\right\|_{B U C\left(0, T^{\prime} ; L^{q}\right)}$ in (2.50) is well-defined if it holds true that $W_{p}^{2-2 / p}(\Omega) \hookrightarrow L^{q}$. At the same time we have to assure that $W^{1, p}(\Omega) \hookrightarrow$ $L^{q^{\prime}}(\Omega)$, since $\partial_{t} \nabla \phi_{2} \in L^{p}\left(W^{1, p}\right)$. The critical cases occur when $p<(n+2) / 2$. In this situation choose $q=n p /(n-2 p+2)$ and $q^{\prime}=n p /(2 p-2)$. So, recalling $p>(n+2) / 3$, it holds that $W_{p}^{2-2 / p}(\Omega) \hookrightarrow L^{q}$ and $W^{1, p}(\Omega) \hookrightarrow L^{q^{\prime}}$ thanks to Sobolev's embedding theorem, hence

$$
\begin{aligned}
& \left\|\left(c_{i}^{*}+\bar{c}_{i}^{2,+}\right) \partial_{t}\left(\nabla \phi_{2}(\hat{c})\right)\right\|_{L^{p}\left(0, T^{\prime} ; L^{p}\right)} \\
& \leq C\left(\left\|c_{i}^{*}\right\|_{B U C\left(0, T^{\prime} ; W_{p}^{2-2 / p}\right)}+\left\|\bar{c}_{i}^{2}\right\|_{B U C\left(0, T^{\prime} ; W_{p}^{2-2 / p}\right)}\right)\left\|\partial_{t}\left(\nabla \phi_{2}(\hat{c})\right)\right\|_{L^{p}\left(0, T^{\prime} ; W^{1, p}\right)} \\
& \leq C(T)\left(\left\|c_{i}^{*}\right\|_{B U C\left(0, T^{\prime} ; W_{p}^{2-2 / p}\right)}+R\right)\|\hat{c}\|_{\mathbb{E}_{T^{\prime}, p}^{c}} .
\end{aligned}
$$

In contrast to the $L^{r}$ - norms in time the appearing $B U C$-norms of $c_{i}^{*}$ will in general not become small as $T^{\prime} \rightarrow 0$. Here we need an additional factor $T^{\prime \alpha}$ which will be provided by estimating the first factor in (2.46) a little more 
carefully as in (2.44). Indeed, we can estimate

$$
\begin{aligned}
& \left\|\left(c_{i}^{*}+\bar{c}_{i}^{2,+}\right) \nabla \phi_{2}(\hat{c})\right\|_{L^{p}\left(0, T^{\prime} ; L^{p}\right)} \\
& \leq\left(\left\|c_{i}^{*}\right\|_{L^{3 p / 2}\left(0, T^{\prime} ; L^{3 p / 2}\right)}+\left\|\bar{c}_{i}^{2,+}\right\|_{L^{3 p / 2}\left(0, T^{\prime} ; L^{3 p / 2}\right)}\right)\left\|\nabla \phi_{2}(\hat{c})\right\|_{L^{3 p}\left(0, T^{\prime} ; L^{3 p}\right)} \\
& \leq C(T) T^{\prime \alpha}\left(\left\|c_{i}^{*}\right\|_{L^{3 p}\left(0, T^{\prime} ; L^{3 p}\right)}+R\right)\|\hat{c}\|_{\mathbb{E}_{T^{\prime}, p}^{c}}
\end{aligned}
$$

for a certain $\alpha>0$. Note that here and in the sequel the appearing $\alpha$ 's at first might be different. At the end, however, we just need one $\alpha>0$ and we can always choose the smallest of finitely many ones. Thus, as for the generic constant $C$, in the sequel $\alpha$ can change from line to line.

From relations (2.46), (2.47), (2.49), (2.50), (2.52), (2.53), and Young's inequality we now obtain

$$
\begin{aligned}
& \left\|\left(c_{i}^{*}+\bar{c}_{i}^{2,+}\right) \nabla \phi_{2}(\hat{c})\right\|_{W_{p}^{1 / 2}\left(0, T^{\prime} ; L^{p}\right)} \\
& \leq C(T) T^{\prime \alpha}\left(\left\|c_{i}^{*}\right\|_{L^{3 p}\left(0, T^{\prime} ; L^{3 p}\right)}+R\right)^{1 / 2} \\
& \quad \cdot\left(\left\|\partial_{t} c_{i}^{*}\right\|_{L^{p}\left(0, T^{\prime} ; L^{p}\right)}+\left\|c_{i}^{*}\right\|_{B U C\left(0, T^{\prime} ; W_{p}^{2-2 / p}\right)}+R\right)^{1 / 2}\|\hat{c}\|_{\mathbb{E}_{T^{\prime}, p}^{c}} \\
& \leq C(T) T^{\prime \alpha}\left(\left\|c_{i}^{*}\right\|_{L^{3 p}\left(0, T^{\prime} ; L^{3 p}\right)}\right. \\
& \left.\quad+\left\|\partial_{t} c_{i}^{*}\right\|_{L^{p}\left(0, T^{\prime} ; L^{p}\right)}+\left\|c_{i}^{*}\right\|_{B U C\left(0, T^{\prime} ; W_{p}^{2-2 / p}\right)}+R\right)\|\hat{c}\|_{\mathbb{E}_{T^{\prime}, p}^{c}} .
\end{aligned}
$$

Collecting (2.45) and (2.54) then gives

$$
\begin{aligned}
& \left\|\left(c_{i}^{*}+\bar{c}_{i}^{2,+}\right) \nabla \phi_{2}(\hat{c})\right\|_{\mathbb{F}_{T^{\prime}, p}^{c, \partial \Omega}} \\
& \leq C(T)\left(\left\|c^{*}\right\|_{L^{3 p / 2}\left(0, T^{\prime} ; W^{1,3 p / 2}\right)}+\left\|c^{*}\right\|_{L^{3 p}\left(0, T^{\prime} ; L^{3 p}\right)}\right. \\
& \left.\quad+\left\|\partial_{t} c^{*}\right\|_{L^{p}\left(0, T^{\prime} ; L^{p}\right)}+T^{\prime \alpha}\left\|c^{*}\right\|_{B U C\left(0, T^{\prime} ; W_{p}^{2-2 / p}\right)}+R\right)\|\hat{c}\|_{\mathbb{E}_{T^{\prime}, p}^{c}} .
\end{aligned}
$$

Now we turn to the second term in (2.43). This term is basically of the same structure as the first term. For its estimation essentially analogous calculations and arguments can be carried out. Thus we will be brief in detail here. Also note that $\nabla \phi_{1}$ has the same regularity in space as $\nabla \phi_{2}\left(c^{*}\right)$ has, but due to its time-independence it can even be treated in an easier way. This is why we leave this term out of the following computation. In fact, by merely interchanging the roles of the factors we obtain similarly to (2.44) and (2.45) that

$$
\begin{aligned}
& \left\|\hat{c}_{i}^{+}\left(\nabla \phi_{2}\left(c^{*}+\bar{c}^{1}\right)\right)\right\|_{L^{p}\left(0, T^{\prime} ; W^{1, p}\right)} \\
& \leq C(T)\left(\left\|\nabla \phi_{2}\left(c^{*}\right)\right\|_{L^{3 p}\left(0, T^{\prime} ; W^{1,3 p}\right)}+R\right)\left\|\hat{c}_{i}\right\|_{\mathbb{E}_{T^{\prime}, p}^{c}} .
\end{aligned}
$$


Accordingly for the norm in $W_{p}^{1 / 2}\left(0, T^{\prime} ; L^{p}\right)$, by performing similar arguments as in (2.46)-(2.54) we deduce

$$
\begin{aligned}
& \left\|\hat{c}_{i}^{+}\left(\nabla \phi_{2}\left(c^{*}+\bar{c}^{1}\right)\right)\right\|_{W_{p}^{1 / 2}\left(0, T^{\prime} ; L^{p}\right)} \\
& \leq C(T) T^{\alpha}\left(\left\|\nabla \phi_{2}\left(c^{*}\right)\right\|_{B U C\left(0, T^{\prime} ; B U C\right)}+\left\|\nabla \phi_{2}\left(c^{*}\right)\right\|_{L^{3 p}\left(0, T^{\prime} ; L^{3 p}\right)}\right. \\
& \left.\quad+\left\|\partial_{t}\left(\nabla \phi_{2}\left(c^{*}\right)\right)\right\|_{L^{p}\left(0, T^{\prime} ; W^{1, p}\right)}+R\right)\|\hat{c}\|_{\mathbb{E}_{T^{\prime}, p}^{c}} .
\end{aligned}
$$

By the fact that $\nabla \phi_{1}$ and $\nabla \phi_{2}\left(c^{*}\right)$ have the same regularity in space, the same terms will appear for $\nabla \phi_{1}$. Since $\nabla \phi_{1}$ is independent of time then by the Sobolev embedding merely a term as $T^{\prime \alpha}\left\|\nabla \phi_{1}\right\|_{W^{1,3 p}}$ remains. Hence, collecting (2.56) and (2.57) gives us

$$
\begin{aligned}
& \left\|\hat{c}_{i}\left(\nabla \phi_{1}+\nabla \phi_{2}\left(c^{*}+\bar{c}^{1}\right)\right)\right\|_{\mathbb{F}_{T^{\prime}, p}^{c, \partial \Omega}} \\
& \quad \leq C(T)\left(R+T^{\prime \alpha}\left[\left\|\nabla \phi_{2}\left(c^{*}\right)\right\|_{B U C\left(0, T^{\prime} ; B U C\right)}+\left\|\nabla \phi_{1}\right\|_{W^{1,3 p}}\right]\right. \\
& \left.\quad+\left\|\nabla \phi_{2}\left(c^{*}\right)\right\|_{L^{3 p}\left(0, T^{\prime} ; W^{1,3 p}\right)}+\left\|\partial_{t}\left(\nabla \phi_{2}\left(c^{*}\right)\right)\right\|_{L^{p}\left(0, T^{\prime} ; W^{1, p}\right)}\right)\|\hat{c}\|_{\mathbb{E}_{T^{\prime}, p}} .
\end{aligned}
$$

Finally, we conclude (2.31) from relations (2.41), (2.43), (2.55) and (2.58).

Having Lemma 2.6 at hand, we are in position to prove Theorem 1.1 .

Proof. (of Theorem 1.1). Recall the solution operator $L$ from (2.24) and its restriction to ${ }_{0} \mathbb{F}_{T^{\prime}, p}$. Its operator norm is denoted by $\|L\|$. Thanks to Lemma 2.6 we can choose first $R>0$ and then $T^{\prime}>0$ small enough such that

$$
\left\|N\left(\bar{u}^{1}, \bar{c}^{1}\right)-N\left(\bar{u}^{2}, \bar{c}^{2}\right)\right\|_{\mathbb{F}_{T^{\prime}, p}} \leq \frac{1}{2\|L\|}\left\|\left(\bar{u}^{1}, \bar{c}^{1}\right)-\left(\bar{u}^{2}, \bar{c}^{2}\right)\right\|_{\mathbb{E}_{T^{\prime}, p}},
$$

for $\left(\bar{u}^{1}, \bar{c}^{1}\right),\left(\bar{u}^{2}, \bar{c}^{2}\right) \in B_{0 \mathbb{E}_{T^{\prime}, p}}(0, R)$. We may assume that $T^{\prime}>0$ was also chosen such that

$$
\|\bar{N}(0,0)\|_{\mathbb{F}_{T^{\prime}, p}}=\left\|N\left(u^{*}, c^{*}\right)\right\|_{\mathbb{F}_{T^{\prime}, p}} \leq \frac{R}{2\|L\|},
$$

since all terms in $N\left(u^{*}, c^{*}\right)$ consist of time integrals over given functions. Now we can apply the contraction mapping theorem. The estimates

$$
\begin{aligned}
\left\|L \bar{N}\left(\bar{u}^{1}, \bar{c}^{1}\right)-L \bar{N}\left(\bar{u}^{2}, \bar{c}^{2}\right)\right\|_{\mathbb{E}_{T^{\prime}, p}} & \leq\|L\|\left\|N\left(\bar{u}^{1}, \bar{c}^{1}\right)-N\left(\bar{u}^{2}, \bar{c}^{2}\right)\right\|_{\mathbb{F}_{T^{\prime}, p}} \\
& \leq \frac{1}{2}\left\|\left(\bar{u}^{1}, \bar{c}^{1}\right)-\left(\bar{u}^{2}, \bar{c}^{2}\right)\right\|_{\mathbb{E}_{T^{\prime}, p}}
\end{aligned}
$$

for $\left(\bar{u}^{1}, \bar{c}^{1}\right),\left(\bar{u}^{2}, \bar{c}^{2}\right) \in B_{0 \mathbb{E}_{T^{\prime}, p}}(0, R)$ and

$$
\|L \bar{N}(\bar{u}, \bar{c})\| \leq\|L\|(\|\bar{N}(\bar{u}, \bar{c})-\bar{N}(0,0)\|+\|\bar{N}(0,0)\|)
$$




$$
\begin{aligned}
& \leq\|L\|\left(\frac{1}{2\|L\|}\|(\bar{u}, \bar{c})\|+\frac{R}{2\|L\|}\right) \\
& \leq R
\end{aligned}
$$

for $(\bar{u}, \bar{c}) \in B_{0} \mathbb{E}_{T^{\prime}, p}(0, R)$ assure the existence of a fixed point and hence the existence and uniqueness of a local-in-time solution to $\left(P^{\prime}\right)$. Note that our computation implies also the unique local-in-time existence of strong solutions to the original problem $(P)$, cf. Remark 5. The claimed regularity for the pressure then is obtained as an easy consequence of formula (1.27).

Let us denote the solutions to $\left(P^{\prime}\right)$ and $(P)$ by $\left(u^{a}, c^{a}\right)$ and $(u, c)$ respectively and let $c_{i}^{0} \geq 0$, for $i=1, \ldots, N$. If we can show that for $\left(u^{a}, c^{a}\right)$ we have $c^{a} \geq 0$ almost everywhere, then it is also a solution to $(P)$ and by uniqueness we see that $(u, c)=\left(u^{a}, c^{a}\right)$. Let $c_{i}^{-}=\min \left\{c_{i}, 0\right\}$. Note that

$$
\frac{1}{2} \frac{\mathrm{d}}{\mathrm{d} t}\left\|c_{i}^{a,-}\right\|_{2}^{2}=\int_{\Omega} \partial_{t} c_{i}^{a,-} c_{i}^{a,-} d x=\int_{\Omega} \partial_{t} c_{i}^{a} c_{i}^{a,-} d x=\int_{\Omega} \partial_{t} c_{i}^{a,-} c_{i}^{a} d x .
$$

From the fact that $\partial_{t} c_{i}^{a,-} \in L^{p}\left(0, T^{\prime} ; L^{p}\right)$ and $c_{i}^{a} \in \mathbb{E}_{T^{\prime}, p}^{c}$ it is easy to see using Hölder's inequality and Sobolev's embedding theorem, that the last integral is well-defined. Hence formal multiplication of (1.29l) by $c_{i}^{a,-}$ and integration over $\Omega$ gives

$$
\frac{1}{2} \frac{\mathrm{d}}{\mathrm{d} t}\left\|c_{i}^{a,-}\right\|_{2}^{2}+\int_{\Omega}\left(-D_{i} \nabla c_{i}^{a}-D_{i} z_{i} c_{i}^{a,+} \nabla \phi+c_{i}^{a} u^{a}\right) \nabla c_{i}^{a,-} d x=0
$$

Integration by parts, taking into account (1.311), yields

$$
\frac{1}{2} \frac{\mathrm{d}}{\mathrm{d} t}\left\|c_{i}^{a,-}\right\|_{2}^{2}+D_{i}\left\|\nabla c_{i}^{a,-}\right\|_{2}^{2}=D_{i} z_{i} \int_{\Omega} c_{i}^{a,+} \nabla \phi \cdot \nabla c_{i}^{a,-} d x-\int_{\Omega} c_{i}^{a} u \cdot \nabla c_{i}^{a,-} d x .
$$

The first integral on the right-hand side is zero since $c_{i}^{a,+} \cdot \nabla c_{i}^{a,-}=0$. The second one also vanishes due to solenoidality of $u$, using

$$
\int_{\Omega} c_{i}^{a} u \cdot \nabla c_{i}^{a,-} d x=\frac{1}{2} \int_{\Omega} u \cdot \nabla\left(c_{i}^{a,-}\right)^{2}=0 .
$$

Here we used the fact that $c_{i}^{a} \nabla c_{i}^{a} \in L^{1}\left(0, T^{\prime} ; L^{2}(\Omega)\right)$, to make sure that the integral exists. Finally, integrating (2.59) over $(0, t)$ gives

$$
\left\|c_{i}^{a,-}(t)\right\|_{2}^{2}+2 D_{i} \int_{0}^{t}\left\|\nabla c_{i}^{a,-}\right\|_{2}^{2}=\left\|c_{i}^{0,-}\right\|_{2}^{2} \leq 0
$$

for almost all $t \in(0, T)$. So $c_{i}^{a} \geq 0$ almost everywhere, whence $\left(u^{a}, c^{a}\right)$ and $(u, c)$ coincide. This proves Theorem 1.1.

Remark 7. Note that the solution $(u, c)$ is unique up to the maximal time of existence $T_{\max }$. This can be seen as follows: Let $(u, c),(\hat{u}, \hat{c}) \in \mathbb{E}_{T, p}$ be two solutions with initial value $\left(u^{0}, c^{0}\right) \in \mathbb{X}_{p}$, and let $\left(0, T^{\prime}\right)$ be the maximal time interval such that $(u, c),(\hat{u}, \hat{c})$ coincide, $T^{\prime} \leq T<T_{\max }$. Suppose $T$ could be chosen in such a way that $T^{\prime}<T$. From [2] we have $(u, c),(\hat{u}, \hat{c}) \in B U C\left(0, T^{\prime} ; \mathbb{X}_{p}\right)$, so therefore $\left(u\left(T^{\prime}\right), c\left(T^{\prime}\right)\right)=\left(\hat{u}\left(T^{\prime}\right), \hat{c}\left(T^{\prime}\right)\right)$ 
and applying Theorem 1.1 with initial value $\left(u\left(T^{\prime}\right), c\left(T^{\prime}\right)\right) \in \mathbb{X}_{p}$ implies that $(u, c)$ and $(\hat{u}, \hat{c})$ necessarily coincide on $\left(T^{\prime}, T^{\prime}+\varepsilon\right)$, say; this contradicts the maximality of $T^{\prime}$.

Remark 8. The condition $\xi \in W_{p}^{3-1 / p}(\partial \Omega)$ in Theorem 1.1 can be relaxed to, e.g.,

$$
\xi \in W_{p}^{1+2 n /(3 p)-1 / p}(\partial \Omega) .
$$

It is a straight forward calculation that with this condition the facts that $\nabla \phi_{1} \in B U C(\bar{\Omega})$ and $\nabla^{2} \phi_{1} \in L^{3 p}(\Omega)$ remain valid. Having this information at hand, we see that estimate (2.23) and the reasoning to obtain (2.58) is still true. There are no further points where the regularity of $\phi_{1}$ is used. However, because of the unphysical dependence on the space dimension, we chose to state Theorem 1.1 in the given form.

Corollary 1. Let $(u, c)$ be the local strong solution to $(P)$ from Theorem 1.1 with initial data $\left(u^{0}, c^{0}\right)$ where $c_{i}^{0} \geq 0, i=1, \ldots, N$. Then the initial masses $m_{i}:=\int_{\Omega} c_{i}^{0}(x) d x$ are conserved, i.e.

$$
\left\|c_{i}(t)\right\|_{1}=\left\|c_{i}^{0}\right\|_{1}, \quad t \geq 0, \quad i=1, \ldots N .
$$

Proof. The claim follows from nonnegativity of $c_{i}$ and

$$
\frac{\mathrm{d}}{\mathrm{d} t} \int_{\Omega} c_{i} d x=\int_{\Omega} \partial_{t} c_{i} d x=-\int_{\Omega} \operatorname{div} J_{i} d x=-\int_{\partial \Omega} J_{i} \cdot \nu d x=0, \quad i=1, \ldots N,
$$

due to (1.29) and (1.31).

\section{Global Well-Posedness in two Dimensions}

Before we turn our attention to the estimation of the local solution $(u, c)$ from Theorem 1.1 we collect some known technical results which will be employed in the proofs of global well-posedness and stability. Below we write

$$
\log ^{+} x=\max \{0, \log x\}, \text { for } x>0 .
$$

Lemma 3.1. 10, Lemma 3.2] Let $v \geq 0$ be defined on $\Omega$. Then for any $p \geq 1, \varepsilon>0$ and $0 \leq \alpha<1$, there is a constant $C=C(p, \alpha, \varepsilon, \Omega)>0$ such that

$$
\|v\|_{p} \leq \varepsilon\left\|v\left(\log ^{+} v\right)^{1-\alpha}\right\|_{p}+C .
$$

Lemma 3.2. 10, Lemma 3.3] Let $m \geq 1, q>r \geq 1$ and define

$$
\gamma=\frac{\frac{1}{r}-\frac{1}{q}}{\frac{1}{n}-\frac{1}{m}+\frac{1}{r}} .
$$

Suppose that $0<\gamma<1$. Then there exists $\alpha$ with $\gamma<\alpha<1$ and a constant $C=C(m, n, q, r, \Omega)$ such that for all $v \in W^{1, m}(\Omega)$ with $v \geq 0$

$$
\left\|v\left(\log ^{+} v\right)^{1-\alpha}\right\|_{q} \leq C\left\|v \log ^{+} v\right\|_{r}^{1-\alpha}\left(\|\nabla v\|_{m}^{\gamma}\|v\|_{r}^{\alpha-\gamma}+\|v\|_{1}^{\alpha}\right) .
$$


This result is contained in Lemma 3.3 of [10]. Note that the restriction $r>1$ assumed there is not needed in the proof.

Lemma 3.3. Let $\Omega \subset \mathbb{R}^{n}$ be bounded with smooth boundary. Let $1 \leq q<\infty$ if $n=2$ and $1 \leq q<2 n /(n-2)$ if $n \geq 3$. Then there is a constant $C=C(n, q, \Omega)$ such that

$$
\|v\|_{q} \leq C\left(\|\nabla v\|_{2}^{\alpha}\|v\|_{1}^{1-\alpha}+\|v\|_{1}\right)
$$

for any $v \in W^{1,2}(\Omega)$, where

and $0 \leq \alpha<1$.

$$
\alpha=\frac{1-\frac{1}{q}}{\frac{1}{n}+\frac{1}{2}}
$$

This is a direct consequence of the Gagliardo-Nirenberg inequality without boundary conditions, for a proof see e.g. [27.

For convenience we also state the Poincaré inequality in a form which will be used frequently.

Lemma 3.4. 14, Chapter 5.8, Theorem 1] Let $1 \leq p \leq \infty$ and $\Omega \subset \mathbb{R}^{n}$ be a bounded Lipschitz domain. Then there is a constant $C=C(p, n, \Omega)>0$ such that for any $v \in W^{1, p}(\Omega)$

$$
\|v\|_{p} \leq C\|\nabla v\|_{p}+\|v\|_{1}
$$

We finally recall a "uniform version" of Gronwall's inequality.

Lemma 3.5. [39, Chapter III, Lemma 1.1] Let $f, g, h$ be three nonnegative locally integrable functions on $\left(t_{0}, \infty\right)$ such that $f^{\prime}(t)$ is locally integrable on $\left(t_{0}, \infty\right)$, and which satisfy

$$
\begin{gathered}
f^{\prime}(t) \leq g(t) f(t)+h(t), \quad t \geq t_{0}, \\
\int_{t}^{t+r} f(s) d s \leq a_{1}, \quad \int_{t}^{t+r} g(s) d s \leq a_{2}, \quad \int_{t}^{t+r} h(s) d s \leq a_{3}, \quad t \geq t_{0},
\end{gathered}
$$

where $r, a_{i}$ are positive constants. Then

$$
f(t+r) \leq\left(\frac{a_{1}}{r}+a_{3}\right) \exp \left(a_{2}\right), \quad t \geq t_{0} .
$$

For the case $p=2$ the initial data $\left(u^{0}, c^{0}\right)$ has to satisfy $\left(u^{0}, c^{0}\right) \in$ $W_{0, \sigma}^{1,2}(\Omega) \times W^{1,2}(\Omega)$ for the local strong solution $(u, c)$ to be well-defined by virtue of Theorem 1.1. For simplicity we impose the following additional initial regularity condition on the concentrations for the rest of this article:

$$
c_{i}^{0} \in W^{1,2}(\Omega) \cap L^{\infty}(\Omega) .
$$

Note that the $L^{\infty}$-constraint is not a restriction from the physical point of view, since concentrations of dissolved species are naturally finite. From the mathematical point of view, it is still possible to show that all results concerning global well-posedness and asymptotics in two dimensions presented in this work essentially remain valid, if we allow for initial data $\left(u^{0}, c^{0}\right) \in L_{\sigma}^{2}(\Omega) \times L^{2}(\Omega)$ with $c_{i}^{0} \geq 0$. This will be explained in Remark 13 . 
Definition 3.6 (Global solution). Let $\Omega \subset \mathbb{R}^{n}, n \geq 2$. The function $(u, c):[0, \infty) \rightarrow L_{\sigma}^{2}(\Omega) \times L^{2}(\Omega)$ with $c_{i} \geq 0$ is called global solution to $(P)$, if it satisfies $(P)$ almost everywhere and if for all $T<\infty$ it holds true that

$$
(u, c) \in W^{1,2}\left(0, T ; L_{\sigma}^{2}(\Omega) \times L^{2}(\Omega)\right) \cap L^{2}\left(0, T ; \mathcal{D}\left(A_{S}\right) \times W^{2,2}(\Omega)\right) .
$$

Let from now on $\Omega \subset \mathbb{R}^{2}$ be bounded and smooth. For the proof of Theorem 1.2 we aim for an estimate of type

$$
\|(u, c)\|_{\mathbb{E}_{T, 2}} \leq C(1+T)
$$

with $\mathbb{E}_{T, 2}$ the space of solutions defined right before (2.24). This requires appropriate estimates of the local solution $(u, c)$. Applying energy methods we will derive several uniform-in-time bounds for quantities like $\|u\|_{2}$, $\|c\|_{2}$, etc., e.g. $\|u(t)\|_{2} \leq C$ for $t \geq 0$ and $C>0$ not depending on $t$. A justification for the use of energy methods, i.e. the formal multiplication of certain equations of $(P)$ with quantities like $u, c$ and integration over $\Omega$, can be given by the fact that $(u, c)$ satisfies $(P)$ almost everywhere and the integrals in the calculations being finite. Note at this stage that since we deal with strong solutions these boundedness relations are merely valid in an almost everywhere sense. However, as we aim at controlling the norm in $\mathbb{E}_{T, 2}$, this is sufficient for our purpose.

Let from now on $(u, c) \in \mathbb{E}_{T, 2}$ denote the unique local strong solution from Theorem 1.1 for initial data

$$
\left(u^{0}, c^{0}\right) \in W_{0, \sigma}^{1,2}(\Omega) \times\left(W^{1,2}(\Omega) \cap L^{\infty}(\Omega)\right) .
$$

For $(u, c) \in L_{\sigma}^{2}(\Omega) \times L^{2}(\Omega)$ with $c_{i} \geq 0$ we define the energy functional

$$
E(u, c):=\frac{1}{2}\|u\|_{2}^{2}+\sum_{i=1}^{N} \int_{\Omega} c_{i} \log c_{i} d x+\frac{\varepsilon}{2}\|\nabla \phi\|_{2}^{2}+\frac{\varepsilon \tau}{2}\|\phi\|_{2, \partial \Omega}^{2},
$$

where $\phi$ is the solution to

$$
-\varepsilon \Delta \phi=\sum_{j=1}^{N} z_{j} c_{j}, \quad \partial_{\nu} \phi+\tau \phi=\xi .
$$

Recall that $\xi$ is assumed to be a time-independent function on the surface $\partial \Omega$. Note also that the term $\int_{\Omega} c_{i} \log c_{i} d x$ is finite for $c_{i} \in L^{2}(\Omega)$ with $c_{i} \geq 0$, since

$$
x \log x \leq \begin{cases}0, & x \in(0,1), \\ x^{2}, & x>1 .\end{cases}
$$

In the following we set $(x \log x)_{\mid x=0}:=0$. 
Let $T_{\max }$ be the maximal time of existence of solutions $(u, c)$. For $t \in$ $\left[0, T_{\max }\right)$ we define

$$
\begin{aligned}
V(t) & :=E(u(t), c(t)) \\
& =\frac{1}{2}\|u(t)\|_{2}^{2}+\sum_{i=1}^{N} \int_{\Omega} c_{i}(t) \log c_{i}(t) d x+\frac{\varepsilon}{2}\|\nabla \phi(t)\|_{2}^{2}+\frac{\varepsilon \tau}{2}\|\phi(t)\|_{2, \partial \Omega}^{2} .
\end{aligned}
$$

The term $\int_{\Omega} c_{i} \log c_{i} d x$ is bounded from below, since $x \log x \geq-1$, say, for $x \geq 0$. As the remaining terms in $V$ are nonnegative, $V$ is bounded from below.

Lemma 3.7. Suppose $(u, c)$ is the local strong solution to $(P)$ from Theorem 1.1 .

(a) We have

$$
t \mapsto \int_{\Omega} \frac{1}{D_{i} c_{i}(t)}\left|j_{i}(t)\right|^{2} d x \in L_{l o c}^{1}\left(\left[0, T_{\max }\right)\right),
$$

where $j_{i}=-D_{i} \nabla c_{i}-D_{i} z_{i} c_{i} \nabla \phi$ denotes the flux of species $i$.

(b) The function $V$ from (3.4) is a Lyapunov functional for system (P). More precisely, it holds true that

$$
\frac{\mathrm{d}}{\mathrm{d} t} V(t)=-\|\nabla u(t)\|_{2}^{2}-\sum_{i=1}^{N} \int_{\Omega} \frac{1}{D_{i} c_{i}(t)}\left|j_{i}(t)\right|^{2} d x \leq 0
$$

for almost all $t \in\left(0, T_{\max }\right)$.

In the following proof we suppress all arguments of functions under integrals for better readability, whenever this does not lead to misunderstandings.

Proof. (of Lemma 3.7). In view of the derivation of (3.5) and the mere nonnegativity of $c_{i}$ we have to first give a meaning to the term $\int_{\Omega} \frac{1}{D_{i} c_{i}}\left|j_{i}\right|^{2} d x$, since it might not be well-defined. As will become clear in the proof below, this integral is connected to the term $\frac{\mathrm{d}}{\mathrm{d} t} \int_{\Omega} c_{i} \log c_{i} d x$, if it exists. We cannot just interchange differentiation and integration and then differentiate under the integral, since the derivative of the function $x \mapsto x \log x$ tends to $\infty$ for $x \rightarrow 0$. So it is not obvious that the term $\int_{\Omega} c_{i} \log c_{i} d x$ is differentiable with respect to time at all, since we only know $c_{i} \geq 0$. This is the reason why we first consider an approximation of this term. For $1 \leq i \leq N$ and $\delta>0$ let

$$
g_{i, \delta}(t):=\int_{\Omega}\left(c_{i}+\delta\right) \log \left(c_{i}+\delta\right) d x, \quad t \in\left[0, T_{\max }\right) .
$$

Clearly $g_{i, \delta}$ is differentiable with respect to time and we have

$$
\frac{\mathrm{d}}{\mathrm{d} t} g_{i, \delta}(t)=\int_{\Omega} \partial_{t} c_{i} \log \left(c_{i}+\delta\right) d x+\int_{\Omega} \partial_{t} c_{i} d x
$$


The last term is zero by (2.61); with (1.29), (1.31), integration by parts, the notation in (1.35) and straight forward manipulations we obtain

$$
\begin{aligned}
\frac{\mathrm{d}}{\mathrm{d} t} g_{i, \delta}(t)= & -\int_{\Omega}\left(\operatorname{div} J_{i}\right) \log \left(c_{i}+\delta\right) d x=\int_{\Omega} J_{i} \cdot \nabla \log \left(c_{i}+\delta\right) d x \\
= & \int_{\Omega} \frac{1}{c_{i}+\delta} \nabla c_{i} \cdot J_{i} d x \\
= & \int_{\Omega} \frac{1}{D_{i}\left(c_{i}+\delta\right)}\left(D_{i} \nabla c_{i}+D_{i} z_{i} c_{i} \nabla \phi\right) \cdot\left(-D_{i} \nabla c_{i}-D_{i} z_{i} c_{i} \nabla \phi\right) d x \\
& +z_{i} \int_{\Omega} \frac{c_{i}}{c_{i}+\delta} \nabla \phi \cdot\left(D_{i} \nabla c_{i}+D_{i} z_{i} c_{i} \nabla \phi-c_{i} u\right) d x \\
& +\int_{\Omega} \frac{c_{i}}{c_{i}+\delta} u \cdot\left(z_{i} c_{i} \nabla \phi+\nabla c_{i}\right) d x \\
= & -\int_{\Omega} \frac{1}{D_{i}\left(c_{i}+\delta\right)}\left|j_{i}\right|^{2} d x-z_{i} \int_{\Omega} \frac{c_{i}}{c_{i}+\delta} \nabla \phi \cdot J_{i} d x \\
& +z_{i} \int_{\Omega} \frac{c_{i}}{c_{i}+\delta} c_{i} u \cdot \nabla \phi d x+\int_{\Omega} \frac{c_{i}}{c_{i}+\delta} \nabla c_{i} \cdot u d x, \quad t \in\left(0, T_{\max }\right) .
\end{aligned}
$$

Integrating equation (3.7) form 0 to $t \in\left(0, T_{\max }\right)$ yields, after rearrangement,

$$
\begin{aligned}
\int_{0}^{t} \int_{\Omega} \frac{1}{D_{i}\left(c_{i}+\delta\right)}\left|j_{i}\right|^{2} d x=g_{i, \delta}(0)-g_{i, \delta}(t)-z_{i} \int_{0}^{t} \int_{\Omega} \frac{c_{i}}{c_{i}+\delta} \nabla \phi \cdot J_{i} d x d s \\
+z_{i} \int_{0}^{t} \int_{\Omega} \frac{c_{i}}{c_{i}+\delta} c_{i} u \cdot \nabla \phi d x d s+\int_{0}^{t} \int_{\Omega} \frac{c_{i}}{c_{i}+\delta} \nabla c_{i} \cdot u d x d s
\end{aligned}
$$

From this equation we are going to conclude assertion (a). The monotone convergence theorem implies that

$$
\int_{0}^{t} \int_{\Omega} \frac{1}{D_{i} c_{i}}\left|j_{i}\right|^{2} d x=\lim _{\delta \rightarrow 0} \int_{0}^{t} \int_{\Omega} \frac{1}{D_{i}\left(c_{i}+\delta\right)}\left|j_{i}\right|^{2} d x
$$

however this limit might be infinite. To exclude this case we consider $\lim _{\delta \rightarrow \infty}$ of the right-hand side of (3.8). Relation (3.3) and the fact that $x \log x \geq-1$, say, implies

$$
\left|\left(c_{i}+\delta\right) \log \left(c_{i}+\delta\right)\right| \leq 1+\left(c_{i}+\delta\right)^{2}
$$

so there is an integrable majorant due to $c_{i}(t) \in L^{2}(\Omega)$, thus by continuity of $x \mapsto x \log x, x \geq 0$,

$$
\lim _{\delta \rightarrow 0} g_{i, \delta}(t)=\int_{\Omega} c_{i} \log c_{i} d x, \quad t \in\left[0, T_{\max }\right) .
$$

For the remaining terms, note that $c_{i} /\left(c_{i}+\delta\right) \leq 1$, so by

$$
\int_{0}^{t} \int_{\Omega}\left|\nabla \phi \cdot \nabla c_{i}\right| d x d s \leq\|\nabla \phi\|_{L^{2}\left(0, t ; L^{2}\right)}\left\|\nabla c_{i}\right\|_{L^{2}\left(0, t ; L^{2}\right)}<\infty
$$




$$
\begin{aligned}
& \left.\int_{0}^{t} \int_{\Omega}\left|c_{i}\right| \nabla \phi\right|^{2} \mid d x d s \leq\left\|c_{i}\right\|_{L^{2}\left(0, t ; L^{2}\right)}\|\nabla \phi\|_{L^{4}\left(0, t ; L^{4}\right)}<\infty \\
& \int_{0}^{t} \int_{\Omega}\left|c_{i} u \cdot \nabla \phi\right| d x d s \leq\left\|c_{i}\right\|_{L^{2}\left(0, t ; L^{2}\right)}\|u\|_{L^{4}\left(0, t ; L^{4}\right)}\|\nabla \phi\|_{L^{4}\left(0, t ; L^{4}\right)}<\infty \\
& \int_{0}^{t} \int_{\Omega}\left|\nabla c_{i} \cdot u\right| d x d s \leq\left\|\nabla c_{i}\right\|_{L^{2}\left(0, t ; L^{2}\right)}\|u\|_{L^{2}\left(0, t ; L^{2}\right)}<\infty
\end{aligned}
$$

the premises of the dominated convergence theorem are satisfied. Because of $c_{i} /\left(c_{i}+\delta\right) \rightarrow 1$ as $\delta \rightarrow 0$ taking the limit on both sides of equation (3.8) gives

$$
\begin{aligned}
\int_{0}^{t} \int_{\Omega} \frac{1}{D_{i} c_{i}}\left|j_{i}\right|^{2} d x & =\int_{\Omega} c_{i}^{0} \log c_{i}^{0} d x-\int_{\Omega} c_{i}(t) \log c_{i}(t) d x \\
& -z_{i} \int_{0}^{t} \int_{\Omega} \nabla \phi \cdot J_{i} d x d s+z_{i} \int_{0}^{t} \int_{\Omega} c_{i} u \cdot \nabla \phi d x d s<\infty
\end{aligned}
$$

where we used the fact that due to $u \in L_{\sigma}^{2}(\Omega)$ the term $\int_{\Omega} \nabla c_{i} \cdot u d x=0$. Claim (a) is proven.

Relation (3.9) implies that $t \mapsto \int_{\Omega} c_{i} \log c_{i} d x$ is absolutely continuous and its a.e. derivative is given by

$$
\frac{\mathrm{d}}{\mathrm{d} t} \int_{\Omega} c_{i} \log c_{i} d x=-\int_{\Omega} \frac{1}{D_{i} c_{i}}\left|j_{i}\right|^{2} d x-z_{i} \int_{\Omega} \nabla \phi \cdot J_{i} d x+z_{i} \int_{\Omega} c_{i} u \cdot \nabla \phi d x
$$

for almost all $t \in\left(0, T_{\max }\right)$; note that the right-hand side of (3.10) is in $L_{l o c}^{1}\left(0, T_{\max }\right)$ due to (3.9) .

In two dimensions the energy equality for weak solutions of the NavierStokes equations is valid, cf. [37, Theorem V.1.4.2]. Since strong solutions are also weak solutions, we have

$$
\frac{1}{2} \frac{\mathrm{d}}{\mathrm{d} t}\|u\|_{2}^{2}=-\|\nabla u\|_{2}^{2}-\sum_{i=1}^{N} z_{i} \int_{\Omega} c_{i} \nabla \phi \cdot u d x, \quad t \in\left(0, T_{\max }\right) .
$$

Recalling the definition of $V$ in (3.4) with the help of summation of (3.10) over $i$, (3.11) and interchanging differentiation and integration, we obtain

$$
\begin{aligned}
\frac{\mathrm{d}}{\mathrm{d} t} V(t)= & -\|\nabla u\|_{2}^{2}-\sum_{i=1}^{N} \int_{\Omega} \frac{1}{D_{i} c_{i}}\left|j_{i}\right|^{2} d x-\sum_{i=1}^{N} z_{i} \int_{\Omega} \nabla \phi \cdot J_{i} d x \\
& +\varepsilon \int_{\Omega} \nabla \phi \cdot \nabla \phi_{t} d x+\varepsilon \tau \int_{\partial \Omega} \phi \phi_{t} d x
\end{aligned}
$$

for almost all $t \in\left(0, T_{\max }\right)$, where we write $\phi_{t}:=\partial_{t} \phi$. Note that the respective last terms of (3.10) and (3.11) cancel. Observe also that due to the time independence of $\xi$ differentiating the boundary condition of (3.2) 
in time yields $\partial_{\nu} \phi_{t}+\tau \phi_{t}=0$. Hence using integration by parts, taking into account (1.29), (1.31) and the time-independence of $\xi$ gives

$$
\begin{aligned}
\sum_{i=1}^{N} z_{i} \int_{\Omega} \nabla \phi \cdot J_{i} d x & =\int_{\Omega} \phi \partial_{t}\left(\sum_{i=1}^{N} z_{i} c_{i}\right) d x=-\varepsilon \int_{\Omega} \phi \Delta \phi_{t} d x \\
& =\varepsilon \int_{\Omega} \nabla \phi \cdot \nabla \phi_{t} d x-\varepsilon \int_{\partial \Omega} \phi \partial_{\nu} \phi_{t} d x \\
& =\varepsilon \int_{\Omega} \nabla \phi \cdot \nabla \phi_{t} d x+\varepsilon \tau \int_{\partial \Omega} \phi \phi_{t} d x .
\end{aligned}
$$

Thus, collecting (3.12) and (3.13), we see

$$
\frac{\mathrm{d}}{\mathrm{d} t} V(t)=-\|\nabla u\|_{2}^{2}-\sum_{i=1}^{N} \int_{\Omega} \frac{1}{D_{i} c_{i}}\left|j_{i}\right|^{2} d x \leq 0
$$

for almost all $t \in\left(0, T_{\max }\right)$.

Remark 9. Observe that due to $V \in W_{l o c}^{1,1}\left(0, T_{\max }\right)$ the function $V$ is absolutely continuous, so for almost all $t \in\left(0, T_{\max }\right)$ it holds true that

$$
V(t)=V(0)-\int_{0}^{t}\left(\|\nabla u(s)\|_{2}^{2}+\sum_{i=1}^{N} \int_{\Omega} \frac{1}{D_{i} c_{i}(s)}\left|j_{i}(s)\right|^{2} d x\right) d s .
$$

Hence $V$ is non-increasing.

Lemma 3.8. There is a constant $C>0$, depending only on the initial data, such that for almost all $t \in\left(0, T_{\max }\right)$

$$
\|u(t)\|_{2}+\left\|c_{i}(t) \log c_{i}(t)\right\|_{1} \leq C .
$$

Proof. From (3.14) we have $V(t) \leq V(0)$, hence

$$
\|u(t)\|_{2}^{2}+\int_{\Omega} c_{i}(t) \log c_{i}(t) d x \leq C_{0}
$$

where $C_{0}>0$ only depends on the initial data. From $c_{i} \log c_{i} \geq-1$ we deduce

$$
\int_{\Omega}\left|c_{i}(t) \log c_{i}(t)\right| d x \leq \int_{\Omega} c_{i} \log c_{i} d x+2|\Omega| \leq C_{0}^{\prime}
$$

which shows the claim.

With Lemma 3.2 the boundedness of the term $\left\|c_{i} \log c_{i}\right\|_{1}$ enables us to show that the $L^{2}(\Omega)$-norm of concentrations $c_{i}$ remains uniformly bounded as long as the solution exists.

Lemma 3.9. There is a constant $C>0$, depending only on the initial data, such that for almost all $t \in\left(0, T_{\max }\right)$

$$
\left\|c_{i}(t)\right\|_{2} \leq C \quad \text { and } \quad \int_{0}^{t}\left\|\nabla c_{i}(s)\right\|_{2}^{2} d s \leq C(1+t), \quad i=1, \ldots, N .
$$


Proof. We multiply equation (1.29) by $c_{i}$ and integrate over $\Omega$ by parts, making use of (1.31), to get

$$
\frac{1}{2} \frac{\mathrm{d}}{\mathrm{d} t}\left\|c_{i}\right\|_{2}^{2}+D_{i}\left\|\nabla c_{i}\right\|_{2}^{2}=-D_{i} z_{i} \int_{\Omega} c_{i} \nabla \phi \cdot \nabla c_{i} d x+\int_{\Omega} c_{i} u \cdot \nabla c_{i} d x
$$

Since

$$
\int_{\Omega} c_{i} u \cdot \nabla c_{i} d x=\frac{1}{2} \int_{\Omega} u \cdot \nabla\left(c_{i}^{2}\right) d x=0
$$

due to $u \in L_{\sigma}^{2}(\Omega)$ and $\nabla\left(c_{i}^{2}\right) \in L^{2}(\Omega)$, we are left with

$$
\frac{1}{2} \frac{\mathrm{d}}{\mathrm{d} t}\left\|c_{i}\right\|_{2}^{2}+D_{i}\left\|\nabla c_{i}\right\|_{2}^{2} \leq C \int_{\Omega}\left|c_{i} \nabla \phi \cdot \nabla c_{i}\right| d x .
$$

In order to use a Gronwall argument we have to estimate $I_{i}:=\int_{\Omega} \mid c_{i} \nabla \phi$. $\nabla c_{i} \mid d x$ appropriately. For convenience we reproduce the computation from [10] correcting also a slight mistake. We use the same splitting into $\phi_{1}$ and $\phi_{2}$ for the potential $\phi$ as in (2.1) and (2.2). Let $1 \leq q, r<\infty$ be such that $1 / 2=1 / q+1 / r$. Then

$$
I_{i} \leq\left\|\nabla c_{i}\right\|_{2}\left\|c_{i}\right\|_{r}\|\nabla \phi\|_{q} \leq\left\|\nabla c_{i}\right\|_{2}\left\|c_{i}\right\|_{r}\left(\left\|\nabla \phi_{1}\right\|_{q}+\left\|\nabla \phi_{2}\right\|_{q}\right) .
$$

With $W^{2,2}(\Omega) \hookrightarrow W^{1, q}(\Omega)$ for any $q \in[1, \infty)$, since $\Omega \subset \mathbb{R}^{2}$, due to Sobolev's embedding theorem and the help of Remark 2 we compute

$$
\left\|\nabla \phi_{1}\right\|_{q} \leq\left\|\phi_{1}\right\|_{W^{1, q}} \leq C\left\|\phi_{1}\right\|_{W^{2,2}} \leq C^{\prime}\|\xi\|_{W_{2}^{1 / 2}(\partial \Omega)},
$$

hence $\left\|\nabla \phi_{1}\right\|_{q} \leq C$ independent of time. Now let $\delta \in(1,2)$ and $q:=\frac{2 \delta}{2-\delta}$, then by Sobolev's embedding theorem we have $W^{2, \delta}(\Omega) \hookrightarrow W^{1, q}(\Omega)$. So again taking advantage of Remark 2 we have

$$
\left\|\nabla \phi_{2}\right\|_{q} \leq\left\|\phi_{2}\right\|_{W^{1, q}} \leq C\left\|\phi_{2}\right\|_{W^{2, \delta}} \leq C^{\prime} \sum_{j=1}^{N}\left\|c_{j}\right\|_{\delta} .
$$

Combining (3.16)-(3.18) yields

$$
I_{i} \leq C\left\|\nabla c_{i}\right\|_{2}\left\|c_{i}\right\|_{r}\left(1+\sum_{j=1}^{N}\left\|c_{j}\right\|_{\delta}\right),
$$

where the constant $C$ does not depend on $t$ or $c_{i}$.

Applying Lemma 3.1 and Lemma 3.2 and the fact that $\left\|c_{i}\right\|_{1} \equiv m_{i}$ and $\left\|c_{i} \log c_{i}\right\|_{1} \leq C$ due to Corollary 1 and Lemma 3.8, we see that for any $p \geq 1$ and $\varepsilon>0$ there is a constant $C>0$ such that

$$
\left\|c_{i}\right\|_{p} \leq \varepsilon\left\|\nabla c_{i}\right\|_{2}^{1-1 / p}+C
$$


where $C$ depends on $m_{i}$ and the parameters indicated in Lemmas 3.1 and 3.2. Thus we may estimate $I_{i}$ by

$$
\begin{aligned}
I_{i} & \leq C\left\|\nabla c_{i}\right\|_{2}\left(\varepsilon\left\|\nabla c_{i}\right\|_{2}^{1-1 / r}+C\right)\left(\varepsilon\left\|\sum_{j=1}^{N}\right\| \nabla c_{j} \|_{2}^{1-1 / \delta}+C\right) \\
& \leq C\left(\varepsilon\left\|\nabla c_{i}\right\|_{2}^{2-1 / r}\left(1+\sum_{j=1}^{N}\left\|\nabla c_{j}\right\|_{2}^{1-1 / \delta}\right)+\varepsilon \sum_{j=1}^{N}\left\|\nabla c_{j}\right\|_{2}^{1-1 / \delta}+1\right)
\end{aligned}
$$

for $\varepsilon<1$. With the help of

$$
\begin{aligned}
\sum_{i=1}^{N}\left\|\nabla c_{i}\right\|_{2}^{2-1 / r} \sum_{j=1}^{N}\left\|\nabla c_{j}\right\|_{2}^{1-1 / \delta} & \leq C \max _{1 \leq i \leq N}\left\|\nabla c_{i}\right\|_{2}^{3-1 / r-1 / \delta} \\
& \leq C \sum_{i=1}^{N}\left\|\nabla c_{i}\right\|_{2}^{3-1 / r-1 / \delta}
\end{aligned}
$$

and Young's inequality we obtain

$$
\sum_{i=1}^{N} I_{i} \leq C\left(\varepsilon \sum_{i=1}^{N}\left(\left\|\nabla c_{i}\right\|_{2}^{3-1 / r-1 / \delta}+\left\|\nabla c_{i}\right\|_{2}^{2}\right)+1\right) .
$$

From $n=2$ and the definition of $r$ and $\delta$ we see $1 / r+1 / \delta=1$. So, writing $d^{\prime}:=\min \left\{D_{i}\right\}$ and choosing $\varepsilon>0$ small enough, we infer from (3.21)

$$
\sum_{i=1}^{N} I_{i} \leq \frac{d^{\prime}}{2} \sum_{i=1}^{N}\left\|\nabla c_{i}\right\|_{2}^{2}+C
$$

Summing (3.15) over $i$ and using estimate (3.22) yields

$$
\frac{\mathrm{d}}{\mathrm{d} t} \sum_{i=1}^{N}\left\|c_{i}\right\|_{2}^{2} \leq-d^{\prime} \sum_{i=1}^{N}\left\|\nabla c_{i}\right\|_{2}^{2}+C .
$$

Poincaré's inequality (cf. Lemma 3.4) implies that there is a constant $C>0$ such that

$$
-\left\|\nabla c_{i}\right\|_{2} \leq-C\left\|c_{i}\right\|_{2}+C\left\|c_{i}\right\|_{1} \leq-C\left\|c_{i}\right\|_{2}+C^{\prime} .
$$

Plugging this into (3.23) results in

$$
\frac{\mathrm{d}}{\mathrm{d} t} \sum_{i=1}^{N}\left\|c_{i}\right\|_{2}^{2} \leq-d^{\prime \prime} \sum_{i=1}^{N}\left\|c_{i}\right\|_{2}^{2}+C^{\prime} .
$$

With Gronwall's inequality we deduce that there is a $C>0$ such that

$$
\left\|c_{i}(t)\right\|_{2} \leq C, \quad t \in\left[0, T_{\max }\right) .
$$


Integrating (3.23) from 0 to $t$ gives

$$
\int_{0}^{t}\left\|\nabla c_{i}(s)\right\|_{2}^{2} d s \leq C^{\prime}(1+t)
$$

Remark 10. If we integrate (3.23) only over $(t, t+r)$ for $t<T_{\max }$ and $r>0$ small enough such that the integration makes sense, we obtain

$$
\int_{t}^{t+r}\left\|\nabla c_{i}(s)\right\|_{2}^{2} d s \leq C(r)
$$

independently of $t$. This will prove important for the application of the uniform Gronwall inequality in Lemma 3.5.

Remark 11. In three dimensions it is also possible to estimate the term $I_{i}$ along the lines of the proof of Lemma 3.9 using Corollary 1 and Lemmas 3.1 , 3.2 and 3.8. However performing the same calculations one observes that in this case

$$
\frac{1}{r}+\frac{1}{\delta}<1
$$

so $I_{i}$ cannot be absorbed into $\left\|\nabla c_{i}\right\|_{2}^{2}$, cf. [10].

Lemma 3.10. There is a constant $C>0$, depending only on the initial data, such that for almost all $t \in\left(0, T_{\max }\right)$

$$
\left\|c_{i}(t)\right\|_{4}+\|\phi(t)\|_{W^{1, \infty}} \leq C .
$$

Proof. Note that once the uniform boundedness of $\left\|c_{i}\right\|_{4}$ for $t \geq 0$ is obtained, by Sobolev's embedding theorem and Remark 2 it follows that

$$
\|\phi\|_{W^{1, \infty}} \leq C\|\phi\|_{W^{2,4}} \leq C^{\prime}\left(\sum_{j}\left\|c_{j}\right\|_{4}+1\right) \leq C^{\prime \prime} \quad\left(t \in\left(0, T_{\max }\right)\right) .
$$

Let $k \in \mathbb{N}$ and $t \geq 0$. We multiply (1.29) by $c_{i}^{2 k-1}$, integrate over $\Omega$ and by parts and use again the no-flux condition (1.31) to obtain

$$
\begin{aligned}
& \frac{1}{2 k} \frac{\mathrm{d}}{\mathrm{d} t}\left\|c_{i}^{k}\right\|_{2}^{2}=\int_{\Omega} J_{i} \nabla\left(c_{i}^{2 k-1}\right) d x \\
& =(2 k-1) \int_{\Omega}\left(-D_{i} c_{i}^{2 k-2}\left|\nabla c_{i}\right|^{2}-D_{i} z_{i} c_{i}^{2 k-1} \nabla c_{i} \nabla \phi+c_{i}^{2 k-1} u \nabla c_{i}\right) d x \\
& =-D_{i} \frac{(2 k-1)}{k^{2}} \int_{\Omega}\left|\nabla\left(c_{i}^{k}\right)\right|^{2} d x-D_{i} z_{i} \frac{2 k-1}{k} \int_{\Omega} c_{i}^{k} \nabla\left(c_{i}^{k}\right) \nabla \phi d x \\
& \quad+\frac{2 k-1}{2 k} \int_{\Omega} u \nabla\left(c_{i}^{2 k}\right) d x .
\end{aligned}
$$

To make this formal calculation rigorous, it is important to note that $(u, c)$ satisfies problem $(P)$ almost everywhere and that $c_{i} \in$ 
$W_{2}^{1 / 2}\left(0, T ; W^{1,2}(\Omega) \hookrightarrow L^{q}\left(0, T ; L^{q}(\Omega)\right)\right.$ for any $q<\infty$, thus $\left\|c_{i}^{k}\right\|_{2}=\left\|c_{i}\right\|_{2 k}^{k}$ is well-defined for almost all $t \in\left(0, T_{\max }\right)$.

Due to the divergence free condition on $u$ the term $\int_{\Omega} u \cdot \nabla\left(c_{i}^{2 k}\right) d x$ is zero. If we denote

$$
v_{k}:=c_{i}^{k}
$$

we can rewrite (3.25) as

$$
\frac{1}{2 k} \frac{\mathrm{d}}{\mathrm{d} t}\left\|v_{k}\right\|_{2}^{2}=-D_{i} \frac{2 k-1}{k^{2}}\left\|\nabla v_{k}\right\|_{2}^{2}-D_{i} z_{i} \frac{2 k-1}{k} \int_{\Omega} v_{k} \nabla v_{k} \cdot \nabla \phi d x .
$$

The Hölder inequality yields

$$
\frac{1}{2 k} \frac{\mathrm{d}}{\mathrm{d} t}\left\|v_{k}\right\|_{2}^{2} \leq-D_{i} \frac{2 k-1}{k^{2}}\left\|\nabla v_{k}\right\|_{2}^{2}+C \frac{2 k-1}{k}\left\|v_{k}\right\|_{3}\left\|\nabla v_{k}\right\|_{2}\|\nabla \phi\|_{6} .
$$

The uniform boundedness of $\left\|c_{i}\right\|_{2}$ due to Lemma 3.9 and the Sobolev embedding $W^{2,2}(\Omega) \hookrightarrow W^{1,6}(\Omega)$ imply

$$
\|\nabla \phi\|_{6} \leq\|\phi\|_{W^{2,2}} \leq C\left(\sum_{j}\left\|c_{j}\right\|_{2}+1\right) \leq C^{\prime} .
$$

Applying Lemma 3.3 to $\left\|v_{k}\right\|_{3}$ shows that

$$
\left\|v_{k}\right\|_{3} \leq C\left(\left\|\nabla v_{k}\right\|_{2}^{2 / 3}\left\|v_{k}\right\|_{1}^{1 / 3}+\left\|v_{k}\right\|_{1}\right),
$$

where $C>0$ is independent of $v_{k}$ and $t$. Hence we estimate (3.28) further by

$$
\frac{1}{2 k} \frac{\mathrm{d}}{\mathrm{d} t}\left\|v_{k}\right\|_{2}^{2} \leq-\frac{D_{i}}{k}\left\|\nabla v_{k}\right\|_{2}^{2}+C\left\|\nabla v_{k}\right\|_{2}\left(\left\|\nabla v_{k}\right\|_{2}^{2 / 3}\left\|v_{k}\right\|_{1}^{1 / 3}+\left\|v_{k}\right\|_{1}\right) .
$$

To obtain the lemma we set $k=2$; note that $\left\|v_{2}(t)\right\|_{1}=\left\|c_{i}(t)\right\|_{2}^{2} \leq C$, so

$$
\begin{aligned}
\frac{1}{4} \frac{\mathrm{d}}{\mathrm{d} t}\left\|v_{2}\right\|_{2}^{2} & \leq-\frac{D_{i}}{2}\left\|\nabla v_{2}\right\|_{2}^{2}+C^{\prime}\left\|\nabla v_{2}\right\|_{2}\left(\left\|\nabla v_{2}\right\|_{2}^{2 / 3}+1\right) \\
& \leq-\frac{D_{i}}{4}\left\|\nabla v_{2}\right\|_{2}^{2}+C^{\prime \prime}
\end{aligned}
$$

where we made use of Young's inequality in the last step. Applying Poincaré's inequality (Lemma 3.4) and taking into account the boundedness of $\left\|v_{2}\right\|_{1}$ yields constants $d^{\prime}, C>0$, such that

$$
\frac{1}{4} \frac{\mathrm{d}}{\mathrm{d} t}\left\|v_{2}\right\|_{2}^{2} \leq-d^{\prime}\left\|v_{2}\right\|_{2}^{2}+C \text {. }
$$

Finally, from Gronwall's inequality $\left\|v_{2}\right\|_{2}=\left\|c_{i}\right\|_{4}^{2}$ is uniformly bounded for $t \geq 0$, since $\left\|c_{i}(0)\right\|_{4}=\left\|c_{i}^{0}\right\|_{4}<\infty$.

We are now in position to obtain suitable estimates on the velocity field $u$. 
Lemma 3.11. There is a constant $C>0$, depending only on the initial data, such that for almost all $t \in\left(0, T_{\max }\right)$

$$
\int_{0}^{t}\|\nabla u(s)\|_{2}^{2} d s \leq C(1+t)
$$

Proof. Recall the energy equality from (3.11), i.e.

$$
\frac{1}{2} \frac{\mathrm{d}}{\mathrm{d} t}\|u\|_{2}^{2}+\|\nabla u\|_{2}^{2}=-\int_{\Omega} \sum_{i=1}^{N} z_{i} c_{i} \nabla \phi \cdot u d x
$$

The right-hand side can be estimated with the uniform boundedness of $\|u\|_{2}$, $\|c\|_{2}$ and $\|\nabla \phi\|_{\infty}$, cf. Lemmas 3.8, 3.9 and 3.10, as follows:

$$
\left|\int_{\Omega} c_{i} \nabla \phi \cdot u d x\right| \leq\left\|c_{i}\right\|_{2}\|\nabla \phi\|_{\infty}\|u\|_{2} \leq C \quad\left(t \in\left(0, T_{\max }\right)\right) .
$$

So integrating (3.29) from 0 to $t$ in time gives

$$
\frac{1}{2}\|u(t)\|_{2}^{2}+\int_{0}^{t}\|\nabla u(s)\|_{2}^{2} d s \leq \frac{1}{2}\left\|u^{0}\right\|_{2}^{2}+C t \leq C^{\prime}(1+t),
$$

which proves the claim.

Remark 12. Note that if we choose $t$ and $r$ in the same way as in Remark10 and integrate (3.29) over $(t, t+r)$, this results in

$$
\int_{t}^{t+r}\|\nabla u(s)\|_{2}^{2} d s \leq C^{\prime}(r)
$$

where $C^{\prime}$ only depends on $r$, but not on $t$.

We also need estimates on higher derivatives of $u$.

Lemma 3.12. There is a constant $C>0$, depending only on the initial data, such that for almost all $t \in\left(0, T_{\max }\right)$

$$
\|\nabla u(t)\|_{2}^{2} \leq C \quad \text { and } \quad \int_{0}^{t}\left\|A_{S} u(s)\right\|_{2}^{2} d s \leq C(1+t)
$$

where $A_{S}$ denotes the Stokes operator defined in Remark 1 .

Proof. Multiplying (1.28) by $A_{S} u$ and integrating over $\Omega$ yields

$$
\frac{1}{2} \frac{\mathrm{d}}{\mathrm{d} t}\|\nabla u\|_{2}^{2}+\left\|A_{S} u\right\|_{2}^{2}=-\int_{\Omega}(u \cdot \nabla) u \cdot A_{S} u d x-\int_{\Omega} \sum_{i=1}^{N} z_{i} c_{i} \nabla \phi \cdot\left(A_{S} u\right) d x
$$

where we used the well-known fact that

$$
\int_{\Omega} u_{t} \cdot A_{S} u d x=-\int_{\Omega} u_{t} \Delta u d x=\int_{\Omega} \nabla u_{t}: \nabla u-\int_{\partial \Omega} u_{t} \partial_{\nu} u d x=\frac{1}{2} \frac{\mathrm{d}}{\mathrm{d} t}\|\nabla u\|_{2}^{2} .
$$


Working in two dimensions, the Gagliardo-Nirenberg inequality implies

$$
\|v\|_{4} \leq C\|v\|_{2}^{1 / 2}\|\nabla v\|_{2}^{1 / 2}, \quad \text { for } v \in W^{1,2}(\Omega) .
$$

The Stokes operator in $L_{\sigma}^{2}(\Omega), \Omega \subset \mathbb{R}^{n}$ a bounded smooth domain, is closed with $0 \in \rho\left(A_{S}\right)$, cf. [37, Theorem III.2.1.1]. Hence, we infer $\left\|\nabla^{2} u\right\|_{2} \leq$ $\|u\|_{W^{2,2}} \leq C\left\|A_{S} u\right\|_{2}$ for $u \in \mathcal{D}\left(A_{S}\right)$. This in combination with (3.34), the uniform boundedness of $\|u\|_{2}$ by Lemma 3.8, and Young's inequality yield the following estimate on the non-linearity.

$$
\begin{aligned}
\left|\int_{\Omega}(u \cdot \nabla) u \cdot A_{S} u d x\right| & \leq\|u\|_{4}\|\nabla u\|_{4}\left\|A_{S} u\right\|_{2} \\
& \leq C\|u\|_{2}^{1 / 2}\|\nabla u\|_{2}\left\|\nabla^{2} u\right\|_{2}^{1 / 2}\left\|A_{S} u\right\|_{2} \\
& \leq \frac{1}{4}\left\|A_{S} u\right\|_{2}^{2}+C^{\prime}\|\nabla u\|_{2}^{4} .
\end{aligned}
$$

Using the a priori estimates on $c_{i}$ and $\nabla \phi$ from Lemma 3.9 and 3.10 and Young's inequality the nonlinear electro-kinetic part can be treated by

$$
\begin{aligned}
\left|\int_{\Omega} \sum_{i=1}^{N} z_{i} c_{i} \nabla \phi \cdot A_{S} u d x\right| & \leq C\|\nabla \phi\|_{\infty}\|c\|_{2}\left\|A_{S} u\right\|_{2} \\
& \leq \frac{1}{4}\left\|A_{S} u\right\|_{2}^{2}+C^{\prime} .
\end{aligned}
$$

So estimating (3.32) with (3.35) and (3.36) results in

$$
\frac{\mathrm{d}}{\mathrm{d} t}\|\nabla u\|_{2}^{2}+\left\|A_{S} u\right\|_{2}^{2} \leq C\|\nabla u\|_{2}^{4}+C .
$$

Setting $g(t):=C\|\nabla u\|_{2}^{2}$ this implies

$$
\frac{\mathrm{d}}{\mathrm{d} t}\|\nabla u\|_{2}^{2} \leq g(t)\|\nabla u\|_{2}^{2}+C \quad\left(t \in\left(0, T_{\max }\right)\right) .
$$

If we let $f(t)=\|\nabla u\|_{2}^{2}$, and $h(t):=C$, with Remark 12 all premises for the uniform Gronwall inequality Lemma 3.5 are fullfilled, and hence

$$
\|\nabla u\|_{2}^{2} \leq C^{\prime} \quad\left(t \in\left(0, T_{\max }\right)\right) .
$$

Integrating (3.37) over $(0, t)$ therefore yields

$$
\int_{0}^{t}\left\|A_{S} u(s)\right\|_{2}^{2} d s \leq C^{\prime}(1+t) .
$$

The estimation of higher derivatives of $c_{i}$ turns out to pose certain difficulties since a calculation analogous to (3.33), creates, in general, non-vanishing and unpleasant boundary integrals. Indeed it gives

$$
\begin{aligned}
\int_{\Omega} \partial_{t} c_{i} \cdot \Delta c_{i} d x & =-\int_{\Omega} \nabla\left(\partial_{t} c_{i}\right) \cdot \nabla c_{i} d x+\int_{\partial \Omega}\left(\partial_{t} c_{i}\right)\left(\partial_{\nu} c_{i}\right) d x \\
& =-\frac{1}{2} \frac{\mathrm{d}}{\mathrm{d} t}\left\|\nabla c_{i}\right\|_{2}^{2}+\int_{\partial \Omega}\left(\partial_{t} c_{i}\right)\left(\partial_{\nu} c_{i}\right) d x
\end{aligned}
$$


By this fact, we prefer to introduce new variables instead of working with $c_{i}$ directly. In [10] a problem similar to (1.29)-(1.31) is transformed to a problem for the new variable

$$
\zeta_{i}:=c_{i} \exp \left(z_{i} \phi\right)
$$

We will show that $\zeta$ possesses exactly the regularity as desired for $c$ in Theorem 1.1, i.e.

$$
\zeta \in W^{1,2}(\Omega)\left(0, T ; L^{2}(\Omega)\right) \cap L^{2}\left(0, T ; W^{2,2}(\Omega)\right),
$$

cf. Lemma 3.15. For, we plug $c_{i}=\zeta_{i} \exp \left(-z_{i} \phi\right)$ into (1.29)-(1.31) and obtain the following nonlinear heat equation subject to homogeneous Neumann boundary conditions for $\zeta_{i}$ :

$$
\begin{aligned}
\partial_{t} \zeta_{i}-D_{i} \Delta \zeta_{i} & =-D_{i} z_{i} \nabla \phi \cdot \nabla \zeta_{i}+z_{i} \zeta_{i} \phi_{t}+z_{i} \zeta_{i} u \cdot \nabla \phi-u \cdot \nabla \zeta_{i} \\
\partial_{\nu} \zeta_{i} & =0 \quad \text { in } \Omega,
\end{aligned}
$$

for $i=1, \ldots, N$. Those homogeneous Neumann boundary conditions enable us to apply the corresponding relation to (3.33) for $\zeta$, namely

$$
\int_{\Omega} \partial_{t} \zeta_{i} \cdot \Delta \zeta_{i} d x=-\int_{\Omega} \nabla\left(\partial_{t} \zeta_{i}\right) \cdot \nabla \zeta_{i} d x+\int_{\Omega}\left(\partial_{t} \zeta_{i}\right)\left(\partial_{\nu} \zeta_{i}\right) d x=-\frac{1}{2} \frac{\mathrm{d}}{\mathrm{d} t}\left\|\nabla \zeta_{i}\right\|_{2}^{2}
$$

Once we have "good" estimates for higher derivatives of $\zeta$ we will be able to infer the same behaviour for higher derivatives of $c$, since $c$ and $\zeta$ are connected via $\phi$, which already has nice regularity properties.

However, in order to obtain suitable estimates for $\nabla \zeta$ and $\Delta \zeta$ via the energy method, we need to gather more information on the terms on the right-hand side of (3.39). This will be done in two steps.

First we shall prove the existence of a uniform $L^{\infty}$-bound in space and time for concentrations $c_{i}$ (Lemma 3.13). With this information at hand we gain sufficient information on the new term $\phi_{t}$ (Lemma 3.14).

Lemma 3.13. There is a constant $C>0$, depending only on the initial data, such that for almost all $t \in\left(0, T_{\max }\right)$

$$
\left\|c_{i}(t)\right\|_{\infty} \leq C .
$$

The computation for the proof of Lemma 3.13 is essentially contained in [10], for convenience we provide a proof here correcting a minor mistake. The idea is as follows: If we can show that there is a sequence $p_{k} \rightarrow \infty$ with the property that $\operatorname{ess} \sup _{t \in[0, \infty)}\left\|c_{i}(t)\right\|_{p_{k}} \leq C$ independently of $k$, then not only is $c_{i}(t) \in L^{\infty}(\Omega)$ for almost all $t \in[0, \infty)$, but it also holds that $c_{i} \in L^{\infty}([0, \infty) \times \Omega)$. This method is sometimes referred to as "Moser iteration", cf. [43]. 
Proof. (of Lemma 3.13). Recall the notation $v_{k}=c_{i}^{k}$ for $k \in \mathbb{N}$. Taking into account the $L^{\infty}$-bound for $\nabla \phi$, we improve the estimation of (3.27) given in (3.28) and get

$$
\frac{1}{2 k} \frac{\mathrm{d}}{\mathrm{d} t}\left\|v_{k}\right\|_{2}^{2} \leq-\frac{D_{i}}{k}\left\|\nabla v_{k}\right\|_{2}^{2}+C\left\|v_{k}\right\|_{2}\left\|\nabla v_{k}\right\|_{2} .
$$

Thanks to Lemma 3.3 and Young's inequality, for every $\varepsilon>0$ there is a $C(\varepsilon)>0$ such that

$$
\begin{gathered}
\frac{1}{2 k} \frac{\mathrm{d}}{\mathrm{d} t}\left\|v_{k}\right\|_{2}^{2}-\frac{D_{i}}{k}\left\|\nabla v_{k}\right\|_{2}^{2}+C\left\|\nabla v_{k}\right\|_{2}\left(\left\|\nabla v_{k}\right\|_{2}^{1 / 2}\left\|v_{k}\right\|_{1}^{1 / 2}+\left\|v_{k}\right\|_{1}\right) \\
\leq-\frac{D_{i}}{k}\left\|\nabla v_{k}\right\|_{2}^{2}+\varepsilon\left\|\nabla v_{k}\right\|_{2}^{2}+C(\varepsilon)\left\|v_{k}\right\|_{1}^{2} .
\end{gathered}
$$

If we choose $\varepsilon=\frac{D_{i}}{2 k}$, we get

$$
\frac{1}{2 k} \frac{\mathrm{d}}{\mathrm{d} t}\left\|v_{k}\right\|_{2}^{2} \leq-\frac{D_{i}}{2 k}\left\|\nabla v_{k}\right\|_{2}^{2}+C^{\prime}(k)\left\|v_{k}\right\|_{1}^{2}
$$

where $C^{\prime}=C^{\prime}(k)$ can be controlled by a polynomial in $k$. Poincaré's inequality (Lemma 3.4) implies

$$
-\left\|\nabla v_{k}\right\|_{2}^{2} \leq-\frac{1}{C_{P}}\left\|v_{k}\right\|_{2}^{2}+\left\|v_{k}\right\|_{1}^{2}
$$

with a constant $C_{P}>\max \{1,|\Omega|\}$, without loss of generality. Plugging this into (3.42) and multiplying with $2 k$ gives

$$
\frac{\mathrm{d}}{\mathrm{d} t}\left\|v_{k}\right\|_{2}^{2} \leq-\frac{D_{i}}{C_{P}}\left\|v_{k}\right\|_{2}^{2}+\left(2 k C^{\prime}(k)+D_{i}\right)\left\|v_{k}\right\|_{1}^{2}
$$

Considering a fixed $t \in\left(0, T_{\max }\right)$, we define

$$
S_{k}:=\max \left\{\left\|c_{i}(0)\right\|_{\infty}, \underset{0 \leq s \leq t}{\operatorname{ess} \sup _{0 \leq t}}\left(\int_{\Omega} c_{i}^{k}(s, x) d x\right)^{1 / k}\right\} .
$$

Note that we already proved that $S_{1}, S_{2}, S_{4}$ can be bounded independently of $t$.

With the abbreviations $C_{1}=D_{i} / C_{P}$ and $C_{2}(k)=2 k C^{\prime}(k)+D_{i}$ we deduce from equation (3.43) that

$$
\frac{\mathrm{d}}{\mathrm{d} t}\left\|v_{k}(t)\right\|_{2}^{2} \leq-C_{1}\left\|v_{k}(t)\right\|_{2}^{2}+C_{2}(k) S_{k}^{2 k}, \quad 0 \leq t \leq T .
$$

Multiplying with $e^{C_{1} t}$ yields

$$
\frac{\mathrm{d}}{\mathrm{d} t} e^{C_{1} t}\left\|v_{k}(t)\right\|_{2}^{2} \leq C_{2}(k) S_{k}^{2 k} e^{C_{1} t} .
$$

By a standard Gronwall argument we therefore can achieve that

$$
\begin{aligned}
\left\|v_{k}(t)\right\|_{2}^{2} & \leq\left\|v_{k}(0)\right\|_{2}^{2}+\frac{C_{2}(k)}{C_{1}} S_{k}^{2 k} \leq|\Omega|\left\|c_{i}^{0}\right\|_{\infty}^{2 k}+\frac{C_{2}(k) S_{k}^{2 k}}{C_{1}} \\
& \leq\left(|\Omega|+\frac{C_{2}(k)}{C_{1}}\right) S_{k}^{2 k} \leq \frac{2 C_{2}(k)}{C_{1}} S_{k}^{2 k}, \quad 0 \leq t \leq T_{\max }
\end{aligned}
$$


since we assumed that $C_{P}>|\Omega|$. Therefore

$$
\left(\int_{\Omega} c_{i}(t, x)^{2 k} d x\right)^{1 / 2 k}=\left(\left\|v_{k}(t)\right\|_{2}^{2}\right)^{1 / 2 k} \leq\left(\frac{2 C_{2}(k)}{C_{1}}\right)^{1 / 2 k} S_{k} .
$$

We can find constants $C, m>0$ such that

$$
\frac{2 C_{2}(k)}{C_{1}}=2 C_{P}\left(1+2 k \frac{C^{\prime}(k)}{D_{i}}\right) \leq C k^{m}, \quad k \geq 1 .
$$

Collecting (3.44)-(3.46), we obtain the following recursion relation for $S_{k}$ :

$$
\begin{aligned}
S_{2 k} & \leq \max \left\{\left\|c_{i}(0)\right\|_{\infty},\left(\frac{2 C_{2}(k)}{C_{1}}\right)^{1 / 2 k} S_{k}\right\}=\left(\frac{2 C_{2}(k)}{C_{1}}\right)^{1 / 2 k} S_{k} \\
& \leq C^{1 / 2 k} k^{m / 2 k} S_{k} .
\end{aligned}
$$

Thus from the fact that $S_{4}<\infty$, we deduce that $S_{8}<\infty$ and so on. Note that this recursion is valid for all $0<t<T_{\max }$, and the constant $C$ does neither depend on $t$ nor on $k$.

If we set $k=2^{\mu}, \mu=0,1,2 \ldots$, relation (3.47) implies

$$
S_{2^{\mu+1}} \leq C^{1 / 2^{\mu+1}} 2^{m \mu / 2^{\mu+1}} S_{2^{\mu}}, \quad \mu=0,1,2, \ldots
$$

Since $S_{1}<\infty$ due to Corollary 1 and the series $\sum 2^{-(\mu+1)}$ and $\sum \mu / 2^{\mu+1}$ converge, the sequence $\left(S_{2^{\mu}}\right)_{\mu}$ is bounded. Note that this means that

$$
\operatorname{essiup}_{0 \leq t<T_{\max }}\|c(t)\|_{2^{\mu}} \leq S_{2^{\mu}} \leq C
$$

independently of $\mu$. We therefore proved $\operatorname{ess} \sup _{0 \leq t<T_{\text {max }}}\left\|c_{i}(t)\right\|_{\infty} \leq C$.

Let us consider $\phi_{t}=\partial_{t} \phi$; for almost all $t \in\left(0, T_{\max }\right)$ it holds true that

$$
\begin{aligned}
-\varepsilon \Delta \phi_{t} & =\sum_{i=1}^{N} z_{i} \partial_{t} c_{i}=-\sum_{i=1}^{N} z_{i} \operatorname{div} J_{i}, x \in \Omega, \\
\partial_{\nu} \phi_{t}+\tau \phi_{t} & =0,
\end{aligned}
$$

by interchanging $\partial_{t}$ and $\Delta$ and taking advantage of (1.29) and the timeindependence of $\xi$.

Lemma 3.14. There is a constant $C>0$, depending only on the initial data, such that for almost all $t \in\left(0, T_{\max }\right)$

$$
\left\|\phi_{t}(t)\right\|_{2} \leq C \text {. }
$$

Proof. The proof follows by a duality argument. Let

$$
B h:=-\varepsilon \Delta h, \quad h \in \mathcal{D}(B):=\left\{v \in W^{2,2}(\Omega): \partial_{\nu} v+\tau v=0\right\} .
$$

Remark 2 then implies that $B: \mathcal{D}(B) \rightarrow L^{2}(\Omega)$ is isomorphic. By duality also $B^{*}: L^{2}(\Omega) \rightarrow \mathcal{D}(B)^{*}$ is isomorphic. By the self-adjointness of $B$ and 
since $\mathcal{D}(B)$ is dense in $L^{2}(\Omega)$ we further can regard above $B^{*}$ as the unique extension of $B$, hence we write

$$
B: L^{2}(\Omega) \rightarrow \mathcal{D}(B)^{*} .
$$

According to Theorem 1.1 we have

$$
\operatorname{div} J_{i} \in L^{2}(\Omega) \hookrightarrow \mathcal{D}(B)^{*} .
$$

We now intend to show that the norm of each $\operatorname{div} J_{i}$ in $\mathcal{D}(B)^{*}$ can be estimated uniformly in $t \in\left(0, T_{\max }\right)$. Thanks to (1.31) we can calculate

$$
\begin{aligned}
\left\langle\operatorname{div} J_{i}, h\right\rangle_{\mathcal{D}(B)^{*}, \mathcal{D}(B)} & =\int_{\Omega} h \operatorname{div} J_{i} d x=\int_{\Omega} \nabla h \cdot J_{i} d x \\
& =\int_{\Omega} \nabla h \cdot\left(-D_{i} \nabla c_{i}-D_{i} z_{i} c_{i} \nabla \phi+c_{i} u\right) d x
\end{aligned}
$$

for all $h \in \mathcal{D}(B)$ and for almost all $t \in\left(0, T_{\max }\right)$.

We need to estimate the terms on the right-hand side of (3.50). Applying integration by parts and Hölder's inequality we compute

$$
\begin{aligned}
\left|\int_{\Omega} \nabla h \cdot \nabla c_{i} d x\right| & \leq\left|\int_{\Omega} c_{i} \Delta h d x\right|+\tau\left|\int_{\partial \Omega} c_{i} h d x\right| \\
& \leq\|h\|_{\mathcal{D}(B)}\left\|c_{i}\right\|_{2}+\tau\left\|c_{i}\right\|_{2, \partial \Omega}\|h\|_{2, \partial \Omega} \\
& \leq C\left(\left\|c_{i}\right\|_{2}+\left\|c_{i}\right\|_{\infty}\right)\|h\|_{\mathcal{D}(B)} \\
& \leq C\|h\|_{\mathcal{D}(B)} \quad\left(t \in\left(0, T_{\max }\right)\right)
\end{aligned}
$$

where we made use of the uniform boundedness of $\left\|c_{i}\right\|_{2}$ and $\left\|c_{i}\right\|_{\infty}$, cf. Lemmas 3.9 and 3.13. With the uniform bound on $\|\nabla \phi\|_{\infty}$, cf. Lemma 3.10, we deduce

$$
\left|\int_{\Omega} c_{i} \nabla \phi \cdot \nabla h d x\right| \leq\left\|c_{i}\right\|_{\infty}\|\nabla \phi\|_{\infty}\|h\|_{W^{1,1}} \leq C\|h\|_{\mathcal{D}(B)}
$$

for the second term. Due to Lemma 3.8 the quantity $\|u\|_{2}$ is uniformly bounded in time, so the last term can be estimated by

$$
\left|\int_{\Omega} c_{i} u \cdot \nabla h d x\right| \leq\left\|c_{i}\right\|_{\infty}\|u\|_{2}\|\nabla h\|_{2} \leq C\|h\|_{\mathcal{D}(B)} .
$$

In total we obtain

$$
\left\|\phi_{t}\right\|_{2} \leq \sum_{i=1}^{N} z_{i}\left\|B^{-1} \operatorname{div} J_{i}\right\|_{2} \leq C \sum_{i=1}^{N}\left\|\operatorname{div} J_{i}\right\|_{\mathcal{D}(B)^{*}} \leq C \quad\left(t \in\left(0, T_{\max }\right)\right)
$$

Before treating problem (3.39)-(3.40) let us note the following lemma on the regularity of $\zeta$.

Lemma 3.15. Let $c \in \mathbb{E}_{T, 2}^{c}$ be as in Theorem 1.1 and $\zeta$ be defined as in (3.38). Then it holds true that $\zeta \in \mathbb{E}_{T, 2}^{c}$. 
Proof. This is an immediate consequence of $\phi, \nabla \phi, c_{i}$ and, hence, $\Delta \phi$ being uniformly bounded in $L^{\infty}(\Omega)$ and $\phi_{t}$ being uniformly bounded in $L^{2}(\Omega)$ for any time.

Lemma 3.16. There is a constant $C>0$, depending only on the initial data, such that for almost all $t \in\left(0, T_{\max }\right)$

$$
\left\|\nabla c_{i}(t)\right\|_{2}^{2} \leq C \quad \text { and } \quad \int_{0}^{t}\left\|\Delta c_{i}(s)\right\|_{2}^{2} d s \leq C(1+t) .
$$

Proof. We first derive the corresponding estimate for the transformed variable $\zeta_{i}$, defined in (3.38). We multiply (3.39) with $-\Delta \zeta_{i}$ and get with (3.41)

$$
\begin{aligned}
\frac{1}{2} \frac{\mathrm{d}}{\mathrm{d} t}\left\|\nabla \zeta_{i}\right\|_{2}^{2}+D_{i}\left\|\Delta \zeta_{i}\right\|_{2}^{2}= & z_{i} D_{i} \int_{\Omega} \nabla \phi \cdot \nabla \zeta_{i} \Delta \zeta_{i} d x-z_{i} \int_{\Omega} \zeta_{i} \phi_{t} \Delta \zeta_{i} d x \\
& -z_{i} \int_{\Omega} \zeta_{i} u \cdot \nabla \phi \Delta \zeta_{i} d x+\int_{\Omega} u \cdot \nabla \zeta_{i} \Delta \zeta_{i} d x .
\end{aligned}
$$

Note that the $L^{\infty}$-norm of $\zeta_{i}$ is bounded independently of time, since both $\|c\|_{\infty}$ and $\|\phi\|_{\infty}$ are bounded independently of time, cf. Lemmas 3.10 and 3.13. Estimating the integrals on the right-hand side, apart from the last one, with Hölder's inequality, Lemmas 3.8, 3.12, 3.14, and Young's inequality gives

$$
\begin{aligned}
\left|\int_{\Omega} \nabla \phi \cdot \nabla \zeta_{i} \Delta \zeta_{i} d x\right| & \leq\|\nabla \phi\|_{\infty}\left\|\Delta \zeta_{i}\right\|_{2}\left\|\nabla \zeta_{i}\right\|_{2} \leq \varepsilon_{1}\left\|\Delta \zeta_{i}\right\|_{2}^{2}+C\left(\varepsilon_{1}\right)\left\|\nabla \zeta_{i}\right\|_{2}^{2}, \\
\left|\int_{\Omega} \zeta_{i} \phi_{t} \Delta \zeta_{i} d x\right| & \leq\left\|\zeta_{i}\right\|_{\infty}\left\|\phi_{t}\right\|_{2}\left\|\Delta \zeta_{i}\right\|_{2} \leq \varepsilon_{2}\left\|\Delta \zeta_{i}\right\|_{2}^{2}+C\left(\varepsilon_{2}\right), \\
\left|\int_{\Omega} \zeta_{i} u \cdot \nabla \phi \Delta \zeta_{i} d x\right| & \leq\left\|\zeta_{i}\right\|_{\infty}\|\nabla \phi\|_{\infty}\|u\|_{2}\left\|\Delta \zeta_{i}\right\|_{2} \leq \varepsilon_{3}\left\|\Delta \zeta_{i}\right\|_{2}^{2}+C\left(\varepsilon_{3}\right) .
\end{aligned}
$$

For the remaining integral $\int_{\Omega} u \cdot \nabla \zeta_{i} \Delta \zeta_{i} d x$ recall that if $v \in W^{2,2}(\Omega)$ satisfies homogeneous Neumann boundary conditions, one has $\left\|\nabla^{2} v\right\|_{2} \leq C\|\Delta v\|_{2}$. So Hölder's inequality and the Gagliardo-Nirenberg inequality (3.34) give

$$
\begin{aligned}
& \left|\int_{\Omega} u \cdot \nabla \zeta_{i} \Delta \zeta_{i} d x\right| \leq\|u\|_{4}\left\|\nabla \zeta_{i}\right\|_{4}\left\|\Delta \zeta_{i}\right\|_{2} \\
& \quad \leq C\|u\|_{2}^{1 / 2}\|\nabla u\|_{2}^{1 / 2}\left\|\nabla \zeta_{i}\right\|_{2}^{1 / 2}\left\|\nabla^{2} \zeta_{i}\right\|_{2}^{1 / 2}\left\|\Delta \zeta_{i}\right\|_{2} \leq C^{\prime}\left\|\nabla \zeta_{i}\right\|_{2}^{1 / 2}\left\|\Delta \zeta_{i}\right\|_{2}^{3 / 2} \\
& \quad \leq \varepsilon_{4}\left\|\Delta \zeta_{i}\right\|_{2}^{2}+C\left(\varepsilon_{4}\right)\left\|\nabla \zeta_{i}\right\|_{2}^{2},
\end{aligned}
$$

where we also applied Lemmas 3.8 and 3.12 .

Collecting (3.52) and (3.53) and choosing $\varepsilon_{i}$ small enough we obtain from (3.51)

$$
\frac{\mathrm{d}}{\mathrm{d} t}\left\|\nabla \zeta_{i}\right\|_{2}^{2}+D_{i}\left\|\Delta \zeta_{i}\right\|_{2}^{2} \leq C\left\|\nabla \zeta_{i}\right\|_{2}^{2}+C
$$


It holds that $\nabla \zeta_{i}=\nabla c_{i} \exp \left(z_{i} \phi\right)+z_{i} c_{i} \exp \left(z_{i} \phi\right) \nabla \phi$. Since $\phi$ and $\nabla \phi$ are uniformly bounded in time and space, $\nabla \zeta$ has the same growth as $c_{i}$ in (3.24), namely $\int_{t}^{t+r}\left\|\nabla \zeta_{i}(s)\right\|_{2}^{2} d s \leq C(r), t \geq 0$. Letting $f(t)=\left\|\nabla \zeta_{i}\right\|_{2}^{2}$, $g(t)=h(t)=C$ in the formulation of Lemma 3.5 we find

$$
\left\|\nabla \zeta_{i}\right\|_{2}^{2} \leq C^{\prime} \quad\left(t \in\left(0, T_{\max }\right)\right) .
$$

Integrating (3.54) in time finally gives

$$
\int_{0}^{t}\left\|\Delta \zeta_{i}(s)\right\|_{2}^{2} d s \leq C^{\prime}(1+t)
$$

for some $C^{\prime}>0$.

Since

$$
\begin{aligned}
\nabla c_{i}= & \nabla \zeta_{i} \exp \left(-z_{i} \phi\right)-z_{i} \zeta_{i} \nabla \phi \exp \left(-z_{i} \phi\right) \text { and } \\
\Delta c_{i}= & \Delta \zeta_{i} \exp \left(-z_{i} \phi\right)-2 z_{i} \nabla \zeta_{i} \cdot \nabla \phi \exp \left(-z_{i} \phi\right) \\
& -z_{i} \zeta_{i} \Delta \phi \exp \left(-z_{i} \phi\right)+z_{i}^{2} \zeta_{i}|\nabla \phi|^{2} \exp \left(-z_{i} \phi\right)
\end{aligned}
$$

the estimates (3.55) and (3.56) are also valid for $\nabla c_{i}$ and $\Delta c_{i}$ by the uniform $L^{\infty}$-boundedness of $\phi, \nabla \phi$ and $\zeta_{i}$.

The estimates on $(u, c)$ and on $\phi$ derived so far enable us now to conclude the statement of Theorem 1.2 ,

Proof. (of Theorem 1.2). From Lemmas 3.8, 3.9, 3.12 and 3.16 it follows that

$$
\|u\|_{L^{2}\left(0, T ; \mathcal{D}\left(A_{S}\right)\right)}^{2}+\|c\|_{L^{2}\left(0, T ; W^{2,2}(\Omega)\right)}^{2} \leq C(1+T) .
$$

We use this estimate to show the boundedness of the nonlinearities of $(P)$ in order to prove the boundedness of $\partial_{t}(u, c)$ in $L^{2}\left(0, T ; L^{2}\right)$. We have:

$$
\left\|c_{i} c_{j}\right\|_{L^{2}\left(L^{2}\right)}^{2}+\left\|\nabla c_{i} \cdot \nabla \phi\right\|_{L^{2}\left(L^{2}\right)}^{2} \leq C T \text {. }
$$

For the treatment of the term $u \cdot \nabla c_{i}$ we will, as in the proof of Lemma 3.16, work with the transformed variable $\zeta_{i}$. The reason lies in the homogeneous Neumann conditions for $\zeta_{i}$ which allows for the estimate $\left\|\nabla^{2} \zeta_{i}\right\|_{2} \leq C\left\|\Delta \zeta_{i}\right\|_{2}$ when using the Gagliardo-Nirenberg inequality. Since

$$
u \cdot \nabla c_{i}=u \cdot \nabla \zeta_{i} \exp \left(-z_{i} \phi\right)-z_{i} \zeta_{i} u \cdot \nabla \phi \exp \left(-z_{i} \phi\right),
$$

the estimate

$$
\begin{aligned}
\left\|u \cdot \nabla \zeta_{i}\right\|_{L^{2}\left(L^{2}\right)}^{2} & \leq \int_{0}^{T}\|u\|_{4}^{2}\left\|\nabla \zeta_{i}\right\|_{4}^{2} d t \\
& \leq C \int_{0}^{T}\|u\|_{2}\|\nabla u\|_{2}\left\|\nabla \zeta_{i}\right\|_{2}\left\|\Delta \zeta_{i}\right\|_{2} d t \leq C(1+T),
\end{aligned}
$$

is sufficient to show $\left\|u \cdot \nabla c_{i}\right\|_{L^{2}\left(L^{2}\right)}^{2} \leq C(1+T)$, since $\zeta_{i}, \phi$ and $\nabla \phi$ are uniformly bounded in $L^{\infty}$, cf. Lemmas 3.10 and 3.13, and $u$ is uniformly bounded in $L^{2}$, cf. Lemma 3.8. We infer

$$
\left\|\partial_{t} c_{i}\right\|_{L^{2}\left(L^{2}\right)} \leq C(1+T) .
$$


We proceed in the same way for the Navier-Stokes part, i.e.

$$
\begin{aligned}
\|P(u \cdot \nabla) u\|_{L^{2}\left(L^{2}\right)}^{2} & \leq C \int_{0}^{T}\|u\|_{4}^{2}\|\nabla u\|_{4}^{2} d t \\
& \leq C^{\prime} \int_{0}^{T}\|u\|_{2}\|\nabla u\|_{2}^{2}\left\|\nabla^{2} u\right\|_{2} d t \\
& \leq C^{\prime \prime} \int_{0}^{T}\|u\|_{2}\|\nabla u\|_{2}^{2}\left\|A_{S} u\right\|_{2} d t \leq C^{\prime \prime \prime}(1+T), \\
\left\|\sum_{i=1}^{N} z_{i} c_{i} \nabla \phi\right\|_{L^{2}\left(L^{2}\right)}^{2} & \leq C T
\end{aligned}
$$

where we used the fact that due to $0 \in \rho\left(A_{S}\right)$ we have $\left\|\nabla^{2} u\right\|_{2} \leq C\left\|A_{S} u\right\|_{2}$ for $u \in \mathcal{D}\left(A_{S}\right)$, hence also

$$
\left\|\partial_{t} u\right\|_{L^{2}\left(L^{2}\right)} \leq C(1+T) .
$$

In conclusion it follows from (3.57)-(3.59) that $\|(u, c)\|_{\mathbb{E}_{T, 2}} \leq C(1+T)$ for finite $T$. So $(u, c)$ can be extended to a global strong solution. It is unique by the local uniqueness from Theorem 1.1 .

Remark 13. It is possible to show that a global existence result in the spirit of Theorem 1.2 remains still valid if we only impose $\left(u^{0}, c^{0}\right) \in L_{\sigma}^{2}(\Omega) \times L^{2}(\Omega)$ with $c_{i}^{0} \geq 0$ for the initial data. This will be published in the doctoral thesis of the second author. We only briefly indicate a sketch of the proof.

1. Existence and uniqueness of local-in-time weak solutions $(u, c)$ :

The idea is to define a map $L$ which sends $\bar{c} \in C\left([0, T] ; L^{2}(\Omega)\right) \cap$ $L^{2}\left(0, T ; W^{1,2}(\Omega)\right)$ with $\bar{c}(0)=c^{0}$ to the unique (weak) solution $c \in$ $C\left(0, T ; L^{2}(\Omega)\right) \cap L^{2}\left(0, T ; W^{1,2}(\Omega)\right)$ of

$$
\left.\begin{array}{rl}
\partial_{t} c_{i}+\operatorname{div}\left(-D_{i} \nabla c_{i}+c_{i} \bar{u}\right) & =D_{i} z_{i} \operatorname{div}\left(\bar{c}_{i} \nabla \bar{\phi}\right), \quad i=1, \ldots, N, \\
\left.\partial_{\nu} c_{i}\right|_{\partial \Omega} & =-\left.\left(z_{i} \bar{c}_{i} \partial_{\nu} \bar{\phi}\right)\right|_{\partial \Omega}, \quad i=1, \ldots, N, \\
\left.c_{i}\right|_{t=0} & =c_{i}^{0}, \quad i=1, \ldots, N,
\end{array}\right\}
$$

$i=1, \ldots, N$, where $\bar{\phi}$ is the unique solution to

$$
-\varepsilon \Delta \bar{\phi}=\sum_{j=1}^{N} z_{j} \bar{c}_{j}, \quad \text { in } \Omega, \quad \partial_{\nu} \bar{\phi}+\tau \bar{\phi}=\xi, \quad \text { on } \partial \Omega
$$

and $\bar{u}$ is the unique (weak) solution to

$$
\partial_{t} \bar{u}+A_{S} \bar{u}+P(\bar{u} \cdot \nabla) \bar{u}=-P\left(\sum_{i=1}^{N} z_{i} \bar{c}_{i} \nabla \bar{\phi}\right), \quad \bar{u}(0)=u^{0} .
$$

The unique weak solutions $\bar{u}$ and $c$ exist from well-known results on weak solutions for Navier-Stokes equations, see e.g. [37], and for parabolic equations, see e.g. [27, whereas $\bar{\phi}$ is well-defined by Remark 2] By choosing the 
time $T$ small enough it is possible to show that $L$ is a contraction in a closed subset of

$$
Z_{T}=\left\{v \in C\left([0, T] ; L^{2}(\Omega)\right) \cap L^{2}\left(0, T ; W^{1,2}(\Omega)\right), v(0)=c^{0}\right\} .
$$

Hence $L$ has a unique fixed point, which represents a local-in-time weak solution. This idea has also been used in [35].

2. Usage of regularizing effects of Navier Stokes and parabolic equations:

The goal is to infer that for some positive time the trajectory $(u(\cdot), c(\cdot))$ enters the regularity class for initial data considered in this work, namely $W_{0, \sigma}^{1,2}(\Omega) \times\left(W^{1,2}(\Omega) \cap L^{\infty}(\Omega)\right)$. This can be seen as follows.

Since the weak solution $(u, c)$ is contained in $L^{1}\left(0, T ; W_{0, \sigma}^{1,2}(\Omega) \times W^{1,2}(\Omega)\right)$, by Lebegue's differentiation theorem we obtain that almost every $t \in(0, T)$ is a Lebesgue point for $(u, c)$. Now let $t_{0} \in(0, T)$ be such a Lebesgue point. Then $\left(u\left(t_{0}\right), c\left(t_{0}\right)\right) \in W_{0, \sigma}^{1,2}(\Omega) \times W^{1,2}(\Omega)$ is well-defined. Thanks to Theorem 1.1 there is a unique local strong solution

$$
\left(u^{s}, c^{s}\right) \in W^{1,2}\left(0, T_{0} ; L_{\sigma}^{2}(\Omega) \times L^{2}(\Omega)\right) \cap L^{2}\left(0, T_{0} ; \mathcal{D}\left(A_{S}\right) \times W^{2,2}(\Omega)\right)
$$

with $\left(u^{s}(0), c^{s}(0)\right)=\left(u\left(t_{0}\right), c\left(t_{0}\right)\right)$ and for some $T_{0} \leq T$. Note that strong solutions are also weak solutions, so by uniqueness of weak solutions they must coincide, i.e. $\left(u\left(t_{0}+t\right), c\left(t_{0}+t\right)\right)=\left(u^{s}(t), c^{s}(t)\right)$ for $t \in\left[0, T_{0}\right)$. Therefore $(u(t), c(t)) \in W_{0, \sigma}^{1,2}(\Omega) \times W^{1,2}(\Omega)$ for all $t \in\left(t_{0}, t_{0}+T_{0}\right)$.

It remains to gain $L^{\infty}$-regularity for $c$. To this end, however, we can argue in the same fashion. From

$$
c \in L^{1}\left(t_{0}, t_{0}+T_{0} ; W^{2,2}(\Omega)\right),
$$

we see that $c\left(t_{1}\right) \in W^{2,2}(\Omega) \hookrightarrow L^{\infty}(\Omega)$ for a Lebesgue point $t_{1} \in\left(t_{0}, T_{0}\right)$. Consequently, we have reached the initial situation of this paper.

\section{Steady states}

For the definition of steady states we only require that they solve $\left(P_{s}\right)$ in the weak sense.

Definition 4.1. (Steady state) Let $\Omega \subset \mathbb{R}^{n}, n \geq 2$. The function $(u, c) \in$ $W_{0, \sigma}^{1,2}(\Omega) \times W^{1,2}(\Omega)$ is called a steady state of $(P)$, if $(u, c)$ is a weak solution to $\left(P_{s}\right)$, i.e. if for all $(\varphi, \psi) \in C_{0, \sigma}^{\infty}(\Omega) \times C^{\infty}(\bar{\Omega})$ the following is valid:

$$
\begin{aligned}
-\int_{\Omega} \nabla u \cdot \nabla \varphi d x-\int_{\Omega} u(u \cdot \nabla \varphi) d x & =\sum_{j=1}^{N} z_{j} \int_{\Omega} c_{j} \nabla \phi \cdot \varphi d x \\
\int_{\Omega}\left(-D_{i} \nabla c_{i}-D_{i} z_{i} c_{i} \nabla \phi+c_{i} u\right) \cdot \nabla \psi_{i} d x & =0, \quad i=1, \ldots, N,
\end{aligned}
$$


where $\phi \in W^{3,2}(\Omega)$ satisfies

$$
-\varepsilon \Delta \phi=\sum_{j=1}^{N} z_{j} c_{j} \quad \text { in } \Omega, \quad \partial_{\nu} \phi+\tau \phi=\xi \quad \text { on } \partial \Omega .
$$

It turns out that steady states have in fact better regularity properties as required from Definition 4.1

Lemma 4.2. Let $\left(u^{\infty}, c^{\infty}\right) \in W_{0, \sigma}^{1,2}(\Omega) \times W^{1,2}(\Omega)$ be a steady state of $(P)$. Then $\left(u^{\infty}, c^{\infty}\right) \in W^{2,2}(\Omega) \times W^{2,2}(\Omega)$.

We omit a proof here. It is an easy consequence of a bootstrap argument taking into account the semilinear structure and regularity properties of the Stokes operator and the Neumann Laplacian, cf., e.g., [16], [37], and [40].

Let $\Omega \subset \mathbb{R}^{2}$ be bounded and smooth. Before considering existence and uniqueness of steady states we give a characterization of weak solutions to problem $\left(P_{s}\right)$.

Lemma 4.3. The function $\left(u^{\infty}, c^{\infty}\right) \in W_{0, \sigma}^{1,2}(\Omega) \times W^{1,2}(\Omega)$, where $c_{i}^{\infty} \geq 0$ not identically zero, $i=1, \ldots, N$, is a weak solution to $\left(P_{s}\right)$ if and only if the following conditions are fulfilled:

(i) $u^{\infty}=0$,

(ii) $\zeta_{i}^{\infty}:=c_{i}^{\infty} \exp \left(z_{i} \phi^{\infty}\right) \equiv$ const $, i=1, \ldots, N$,

where $\phi^{\infty}$ is the corresponding electrical potential from 4.3). In this situation there is a constant $\eta_{0}>0$ such that $c_{i}^{\infty} \geq \eta_{0}, i=1, \ldots, N$. The associated pressure $\pi^{\infty}$ satisfies $\pi^{\infty}=\Sigma_{j} c_{j}^{\infty}$ up to some constant.

The proof of this lemma employs the functional $V$ from (3.4). In terms of the transformed variable $\zeta$ from (3.38), the dissipation rate $\frac{\mathrm{d}}{\mathrm{d} t} V(t)$ can be rewritten as

$$
\frac{\mathrm{d}}{\mathrm{d} t} V(t)=-\|\nabla u(t)\|_{2}^{2}-\sum_{i=1}^{N} \int_{\Omega} \frac{D_{i}}{c_{i}} \exp \left(-2 z_{i} \phi(t)\left|\nabla \zeta_{i}\right|^{2} d x,\right.
$$

whenever $(u, c)$ is a global strong solution from Theorem 1.2 .

Proof. (of Lemma 4.3). Suppose conditions $(i)$ and (ii) are satisfied. Since $u^{\infty}=0$ and

$$
0=\nabla \zeta_{i}^{\infty}=\left(\nabla c_{i}^{\infty}+z_{i} c_{i}^{\infty} \nabla \phi^{\infty}\right) \exp \left(z_{i} \phi^{\infty}\right),
$$

we infer that (4.2) is satisfied. From (4.5) it is now evident that the term $-\sum_{j} z_{j} c_{j}^{\infty} \nabla \phi^{\infty}=\nabla \sum_{j} c_{j}^{\infty}$ is a gradient field, so also (4.1) is valid. From this the last assertion of this lemma concerning the pressure follows, too. Observe that $\zeta_{i}^{\infty}=c_{i}^{\infty} \exp \left(z_{i} \phi^{\infty}\right)>0$, because $c_{i} \geq 0$ and $c_{i} \not \equiv 0$. The fact that $\phi^{\infty} \in W^{3,2}(\Omega) \hookrightarrow B U C(\bar{\Omega})$ due to Sobolov's embedding theorem shows that there is a constant $\eta_{0}>0$ such that $c_{i}^{\infty} \geq \eta_{0}, i=1, \ldots, N$. 
Now suppose $\left(u^{\infty}, c^{\infty}\right)$ is a steady state to $(P)$. From Lemma 4.2 the function $\left(u^{s}(t), c^{s}(t)\right):=\left(u^{\infty}, c^{\infty}\right), t \geq 0$, is a global strong solution to $(P)$ in the sense of Definition 3.6. For this stationary solution we have $V(t) \equiv E\left(u^{\infty}, c^{\infty}\right)$, hence $\frac{\mathrm{d}}{\mathrm{d} t} V(t) \equiv 0$. On the other hand, with (4.4) we deduce $\nabla u^{\infty}=0$ and $\nabla \zeta_{i}^{\infty}=0$, which results in $\zeta_{i}^{\infty}=$ const and $u^{\infty}=0$ because of $u^{\infty}{ }_{\mid \partial \Omega}=0$.

In order to make use of the global strong solutions $(u, c)$ from Theorem 1.2 it is crucial to know that the dissipation terms in (4.4) become small as time tends to infinity.

Lemma 4.4. Let $(u, c)$ be a global strong solution to $(P)$ from Theorem 1.2 and $\zeta_{i}=c_{i} \exp \left(z_{i} \phi\right)$ be defined as above. Then

$$
\lim _{t \rightarrow \infty}\left(\|\nabla u(t)\|_{2}^{2}+\left\|\nabla \zeta_{i}(t)\right\|_{2}^{2}\right)=0 .
$$

Proof. From (4.4) and the uniform boundedness of $\|\phi\|_{\infty}$ and $\|c\|_{\infty}$, cf. Lemmas 3.10 and 3.13, we have

$$
\begin{aligned}
\frac{\mathrm{d}}{\mathrm{d} t} V(t) & \leq-\|\nabla u\|_{2}^{2}-\sum_{i=1}^{N} \frac{1}{\left\|c_{i}\right\|_{\infty}\left\|\exp \left(-2 z_{i} \phi\right)\right\|_{\infty}}\left\|\nabla \zeta_{i}\right\|_{2}^{2} \\
& \leq-C\left(\|\nabla u\|_{2}^{2}+\sum_{i=1}^{N}\left\|\nabla \zeta_{i}\right\|_{2}^{2}\right) .
\end{aligned}
$$

The functional $V$ is bounded from below, so using the fact that it is absolutely continuous, cf. Remark 9, we compute

$$
L \leq V(t) \leq V(0)-C \int_{0}^{t}\left(\|\nabla u\|_{2}^{2}+\sum_{i=1}^{N}\left\|\nabla \zeta_{i}\right\|_{2}^{2} d s\right), \quad t \geq 0 .
$$

Rearrangement therefore yields

$$
\int_{0}^{\infty}\left(\|\nabla u\|_{2}^{2}+\sum_{i=1}^{N}\left\|\nabla \zeta_{i}\right\|_{2}^{2}\right) d s<\infty .
$$

From (3.37) there is a constant $C>0$ such that

$$
\frac{\mathrm{d}}{\mathrm{d} t}\|\nabla u\|_{2}^{2} \leq C\|\nabla u\|_{2}^{4}+C .
$$

For $\varepsilon>0$ there is $T_{\varepsilon}>0$ such that

$$
\int_{T_{\varepsilon}}^{\infty}\|\nabla u\|_{2}^{2} d s<\varepsilon
$$

by (4.6). With $f(t):=\|\nabla u\|_{2}^{2}, g(t):=C\|\nabla u\|_{2}^{2}, h(t):=C, a_{1}=\varepsilon, a_{2}=C \varepsilon$ and $a_{3}=C r$ application of Lemma 3.5 yields

$$
\|\nabla u(t+r)\|_{2}^{2} \leq(\varepsilon / r+C r) \exp (C \varepsilon), \quad t \geq T_{\varepsilon} .
$$


So choosing first $r>0$ and then $\varepsilon>0$ small enough shows $\|\nabla u(t)\|_{2}^{2}<\delta$ for $t \geq T_{\varepsilon}+r=: T_{\delta}$. Note that from Remark 10, the uniform bounds on $\phi$ and $\nabla \phi$, and (3.54) we have exactly the same situation for $\nabla \zeta_{i}$ as for $\nabla u$. So the assertion for $\nabla \zeta_{i}$ is obtained in the same way.

Proof. (of Theorem 1.3). Existence.

Let $\left(u^{0}, c^{0}\right) \in W^{1,2}(\Omega) \times\left(W^{1,2}(\Omega) \cap L^{\infty}(\Omega)\right)$ with $c_{i}^{0} \geq 0$ and $\int_{\Omega} c_{i}^{0}(x) d x=$ $m_{i}>0$, let $(u, c)$ be the corresponding global strong solution from Theorem 1.2 and let $\left(t_{k}\right)_{k}$ be a sequence with $t_{k} \rightarrow \infty$. The estimates derived in Section 3 show that for the trajectory $(u, c)$ there is a constant $C$ such that

$$
\left\|u\left(t_{k}\right)\right\|_{2}+\left\|\nabla u\left(t_{k}\right)\right\|_{2}+\left\|c\left(t_{k}\right)\right\|_{2}+\left\|\nabla c_{i}\left(t_{k}\right)\right\|_{2} \leq C
$$

independently of time, cf. Lemmas 3.8, 3.9, 3.12 and 3.16. So $\left(u\left(t_{k}\right), c\left(t_{k}\right)\right)$ is bounded in $W_{0, \sigma}^{1,2}(\Omega) \times W^{1,2}(\Omega)$. By the compact embedding $W^{1,2}(\Omega) \hookrightarrow$ $L^{2}(\Omega)$, the trajectory is relatively compact in $L^{2}(\Omega)$, so there is a subsequence of $\left(t_{k}\right)_{k \in \mathbb{N}}$, which will be denoted in the same way, and an element $\left(u^{\infty}, c^{\infty}\right) \in L_{\sigma}^{2}(\Omega) \times L^{2}(\Omega)$ such that

$$
\left(u\left(t_{k}\right), c\left(t_{k}\right)\right) \rightarrow\left(u^{\infty}, c^{\infty}\right) \text { in } L^{2} .
$$

This convergence implies convergence almost everywhere in $\Omega$ of a subsequence, thus by nonnegativity of $c_{i}\left(t_{k}\right)$ we deduce nonnegativity of $c_{i}^{\infty}$. Since $\left\|c_{i}\left(t_{k}\right)\right\|_{1}=m_{i}$ we readily approve $\left\|c_{i}^{\infty}\right\|_{1}=m_{i}, i=1, \ldots, N$. The convergence of $c_{i}\left(t_{k}\right)$ in $L^{2}$ implies convergence of $\phi\left(t_{k}\right) \rightarrow \phi^{\infty}$ in $W^{2,2}(\Omega)$ where $\phi^{\infty}$ denotes the unique solution to

$$
-\varepsilon \Delta \phi=-\sum_{i=1}^{N} z_{i} c_{i}^{\infty}, \quad \partial_{\nu} \phi+\tau \phi=\xi .
$$

With Poincaré's inequality we obtain

$$
\left\|u\left(t_{k}\right)\right\|_{2} \leq C\left\|\nabla u\left(t_{k}\right)\right\|_{2} \rightarrow 0,
$$

due to Lemma 4.4, so $u^{\infty}=0$.

Recall the definition $\zeta_{i}=c_{i} \exp \left(z_{i} \phi\right)$. From the uniform $L^{\infty}$-bounds on $\phi$ and $c$, cf. Lemmas 3.10 and 3.13, we see that the sequence $\left(\zeta_{i}\left(t_{k}\right)\right)_{k}$ is bounded in $L^{2}(\Omega)$. Since $\nabla \zeta_{i}\left(t_{k}\right) \rightarrow 0$ in $L^{2}(\Omega)$, cf. Lemma 4.4, we infer that $\zeta_{i}$ is bounded in $W^{1,2}(\Omega)$. Since the embedding $W^{1,2}(\Omega) \hookrightarrow L^{2}(\Omega)$ is compact there exists $\zeta_{i}^{\infty} \in L^{2}(\Omega)$ such that, up to a subsequence, $\zeta_{i}\left(t_{k}\right) \rightarrow$ $\zeta_{i}^{\infty}$ in $L^{2}(\Omega)$. Because $\nabla \zeta_{i}\left(t_{k}\right) \rightarrow 0$ we deduce that $\zeta_{i}^{\infty}$ is in fact constant and that $\zeta_{i}\left(t_{k}\right) \rightarrow \zeta_{i}^{\infty}$ in $W^{1,2}(\Omega)$.

From the uniform $L^{\infty}$-bounds of $\phi\left(t_{k}\right)$ and $c_{i}\left(t_{k}\right)$ it follows that $\sum_{j}\left\|c_{j}^{\infty}\right\|_{\infty}+\left\|\phi^{\infty}\right\|_{\infty} \leq C$. Thus from the continuity of the function $(x, y) \mapsto x \exp \left(z_{i} y\right)$ we have $\zeta_{i}^{\infty}=c_{i}^{\infty} \exp \left(z_{i} \phi^{\infty}\right)$ almost everywhere.

Hence if we can show that $c^{\infty} \in W^{1,2}(\Omega)$, then Lemmas 4.2 and 4.3 imply that $\left(u^{\infty}, c^{\infty}\right)$ is a solution to $\left(P_{s}\right)$. 
Note that from the definition of $\zeta_{i}$ we have

$$
\exp \left(-z_{i} \phi\right) \nabla \zeta_{i}=\nabla c_{i}+z_{i} c_{i} \nabla \phi .
$$

Since $\nabla \zeta_{i}(t) \rightarrow 0$ in $L^{2}(\Omega)$ as $t \rightarrow \infty$ and the sequence $\phi\left(t_{k}\right)$ is uniformly bounded in $L^{\infty}(\Omega)$, for the proof of $\nabla c_{i}^{\infty} \in L^{2}$ it is sufficient to show the convergence of $c_{i}\left(t_{k}\right) \nabla \phi\left(t_{k}\right)$ in $L^{2}$. We compute

$$
\begin{aligned}
& \left\|c_{i}\left(t_{k}\right) \nabla \phi\left(t_{k}\right)-c_{i}^{\infty} \nabla \phi^{\infty}\right\|_{2} \\
& \quad \leq\left\|c_{i}\left(t_{k}\right)-c_{i}^{\infty}\right\|_{2}\left\|\nabla \phi\left(t_{k}\right)\right\|_{\infty}+\left\|c_{i}^{\infty}\right\|_{\infty}\left\|\nabla \phi\left(t_{k}\right)-\nabla \phi^{\infty}\right\|_{2} \rightarrow 0,
\end{aligned}
$$

as $k \rightarrow \infty$, where we use the convergence of $\phi\left(t_{k}\right) \rightarrow \phi^{\infty}$ in $W^{1,2}(\Omega)$ and the convergence of $c_{i}\left(t_{k}\right) \rightarrow c_{i}^{\infty}$ in $L^{2}(\Omega)$. So by (4.7) we see that $\nabla c_{i}\left(t_{k}\right)$ converges in $L^{2}(\Omega)$ as well, therefore $c_{i}^{\infty} \in W^{1,2}(\Omega)$ and $\left(u^{\infty}, c^{\infty}\right)=\left(0, c^{\infty}\right)$ is a steady state to problem $(P)$. Therefore $c^{\infty} \in W^{2,2}(\Omega)$ is a solution to $\left(P_{s}^{\prime}\right)$.

Note that the assertion concerning the pressure $\pi^{\infty}$ is already contained in Lemma 4.3

\section{Uniqueness.}

Due to the fact that $u^{\infty}=0$ it is sufficient to prove that $c^{\infty}$ with corresponding $\phi^{\infty}$ is the unique nonnegative solution to $\left(P_{s}^{\prime}\right)$. So suppose there are two nonnegative solutions $c^{\infty}, \tilde{c}^{\infty}$ with $\int_{\Omega} c_{i}^{\infty} d x=\int_{\Omega} \tilde{c}_{i}^{\infty} d x=m_{i}$, $i=1, \ldots, N$. Note that $\int_{\Omega} c_{i}^{\infty}-\tilde{c}_{i}^{\infty} d x=m_{i}-m_{i}=0$. Let us denote the corresponding potentials by $\phi^{\infty}, \tilde{\phi}^{\infty}$. From Lemma 4.3 we have $\zeta_{i}^{\infty}=$ $c_{i}^{\infty} \exp \left(z_{i} \phi^{\infty}\right)=$ const and $\tilde{\zeta}_{i}^{\infty}=\tilde{c}_{i}^{\infty} \exp \left(z_{i} \tilde{\phi}^{\infty}\right)=$ const. Since $\zeta_{i}^{\infty}, \tilde{\zeta}_{i}^{\infty}>0$, we can take the $\operatorname{logarithm}$. It holds $\log \zeta_{i}^{\infty}=\log c_{i}^{\infty}+z_{i} \phi^{\infty}=$ const and correspondingly for $\tilde{\zeta}_{i}^{\infty}$. Therefore, integrating by parts we obtain

$$
\begin{aligned}
0= & \sum_{i=1}^{N} \int_{\Omega}\left(c_{i}^{\infty}-\tilde{c}_{i}^{\infty}\right)\left(\log c_{i}^{\infty}-\log \tilde{c}_{i}^{\infty}+z_{i}\left(\phi^{\infty}-\tilde{\phi}^{\infty}\right)\right) d x \\
= & \int_{\Omega} \sum_{i}\left(z_{i}\left(c_{i}^{\infty}-\tilde{c}_{i}^{\infty}\right)\left(\phi^{\infty}-\tilde{\phi}^{\infty}\right)+\left(c_{i}^{\infty}-\tilde{c}_{i}^{\infty}\right) \log \frac{c_{i}^{\infty}}{\tilde{c}_{i}^{\infty}}\right) d x \\
= & \varepsilon\left\|\nabla\left(\phi^{\infty}-\tilde{\phi}^{\infty}\right)\right\|_{2}^{2}+\varepsilon \tau\left\|\phi^{\infty}-\tilde{\phi}^{\infty}\right\|_{2, \partial \Omega}^{2} \\
& \quad+\int_{\Omega} \sum_{i}\left(c_{i}^{\infty}-\tilde{c}_{i}^{\infty}\right) \log \frac{c_{i}^{\infty}}{\tilde{c}_{i}^{\infty}} d x \geq 0,
\end{aligned}
$$

so $c^{\infty}=\tilde{c}^{\infty}$ and therefore $\phi^{\infty}=\tilde{\phi}^{\infty}$.

\section{Convergence}

It was shown that semi-orbits $(u, c)$ are compact and any accumulation point $\left(u^{\infty}, c^{\infty}\right)$ represents a steady state. The convergence $\left(u\left(t_{k}\right), c\left(t_{k}\right)\right) \rightarrow$ $\left(u^{\infty}, c^{\infty}\right)$ in this situation means convergence with respect to the topology in $W_{0, \sigma}^{1,2}(\Omega) \times W^{1,2}(\Omega)$. From the existence and uniqueness of steady states and the fact that the sequence $\left(t_{k}\right)_{k \in \mathbb{N}}$ was arbitrary, the convergence (1.38) follows. 


\section{Asymptotic Behaviour}

Let $\Omega \subset \mathbb{R}^{2}$ be bounded and smooth, let $\left(u^{0}, c^{0}\right) \in W_{0, \sigma}^{1,2}(\Omega) \times\left(W^{1,2}(\Omega) \cap\right.$ $\left.L^{\infty}(\Omega)\right)$ with $c_{i}^{0} \geq 0$ and corresponding masses $m_{i}=\int_{\Omega} c_{i}^{0} d x>0, i=$ $1, \ldots, N$, be given and let $c^{\infty} \in W^{2,2}(\Omega)$ denote the unique solution to $\left(P_{s}^{\prime}\right.$ subject to $\int_{\Omega} c_{i}^{\infty} d x=m_{i}, i=1, \ldots, N$ from Theorem 1.3 .

Let us define

$$
\mathcal{S}:=W_{0, \sigma}^{1,2}(\Omega) \times\left\{v \in W^{1,2}(\Omega) \cap L^{\infty}(\Omega): v_{i} \geq 0, \int_{\Omega} v_{i}(x) d x=m_{i}\right\}
$$

and introduce the function $\Psi: \mathcal{S} \rightarrow \mathbb{R}$, given by

$$
\begin{aligned}
& \Psi(u, c):=E(u, c)-E\left(u^{\infty}, c^{\infty}\right) \\
& =\frac{1}{2}\|u\|_{2}^{2}+\sum_{i=1}^{N} \int_{\Omega}\left(c_{i} \log c_{i}-c_{i}^{\infty} \log c^{\infty}\right) d x \\
& +\frac{\varepsilon}{2}\left(\|\nabla \phi\|_{2}^{2}-\left\|\nabla \phi^{\infty}\right\|_{2}^{2}\right)+\frac{\varepsilon \tau}{2}\left(\|\phi\|_{2, \partial \Omega}^{2}-\left\|\phi^{\infty}\right\|_{2, \partial \Omega}^{2}\right) \\
& =\frac{1}{2}\|u\|_{2}^{2}+\sum_{i=1}^{N} \int_{\Omega}\left(c_{i}\left(\log \frac{c_{i}}{c_{i}^{\infty}}-1\right)+c_{i}^{\infty}\right) d x+\frac{\varepsilon}{2}\left\|\nabla\left(\phi-\phi^{\infty}\right)\right\|_{2}^{2} \\
& +\frac{\varepsilon \tau}{2}\left\|\phi-\phi^{\infty}\right\|_{2, \partial \Omega}^{2}+\varepsilon \int_{\Omega} \nabla \phi^{\infty} \cdot \nabla\left(\phi-\phi^{\infty}\right) d x \\
& +\varepsilon \tau \int_{\partial \Omega} \phi^{\infty}\left(\phi-\phi^{\infty}\right) d x+\sum_{i=1}^{N} \int_{\Omega}\left(c_{i}-c_{i}^{\infty}\right)\left(1+\log c_{i}^{\infty}\right) d x .
\end{aligned}
$$

Using integration by parts and the fact that $c_{i}^{\infty} \exp \left(z_{i} \phi^{\infty}\right)$ is constant we obtain

$$
\begin{aligned}
& \varepsilon \int_{\Omega} \nabla \phi^{\infty} \cdot \nabla\left(\phi-\phi^{\infty}\right) d x+\varepsilon \tau \int_{\partial \Omega} \phi^{\infty}\left(\phi-\phi^{\infty}\right) d x \\
& \quad+\sum_{i=1}^{N} \int_{\Omega}\left(c_{i}-c_{i}^{\infty}\right)\left(1+\log c_{i}^{\infty}\right) d x \\
& =\int_{\Omega} \sum_{i=1}^{N}\left(c_{i}-c_{i}^{\infty}\right)\left(1+\log c_{i}^{\infty}+z_{i} \phi^{\infty}\right) d x \\
& =\int_{\Omega} \sum_{i=1}^{N}\left(c_{i}-c_{i}^{\infty}\right)\left(1+\log \zeta_{i}^{\infty}\right) d x=0 .
\end{aligned}
$$

Therefore

$$
\begin{aligned}
\Psi(u, c)= & \frac{1}{2}\|u\|_{2}^{2}+\sum_{i=1}^{N} \int_{\Omega}\left(c_{i}\left(\log \frac{c_{i}}{c_{i}^{\infty}}-1\right)+c_{i}^{\infty}\right) d x \\
& +\frac{\varepsilon}{2}\left\|\nabla\left(\phi-\phi^{\infty}\right)\right\|_{2}^{2}+\frac{\varepsilon \tau}{2}\left\|\phi-\phi^{\infty}\right\|_{2, \partial \Omega}^{2} .
\end{aligned}
$$


Let $(u, c)$ be the global strong solution to $(P)$ from Theorem 1.2 with initial data $\left(u^{0}, c^{0}\right)$. Note that due to Theorem 1.1 and Corollary 1 for almost all $t \geq 0$ we have $(u(t), c(t)) \in \mathcal{S}$, hence $t \mapsto \Psi(u(t), c(t))$ is well-defined. The value $\Psi(u(t), c(t))$ represents the difference between the energy at some time $t$ and the energy in the equilibrium state. From the monotone behaviour of $V$ along solutions of $(P)$, cf. Lemma 3.7, we see that $\Psi$ is monotonely decreasing as well with the same dissipation rate given in (4.4).

Proposition 1. Let $a, b>0$.

(a) It holds

$$
(\sqrt{a}-\sqrt{b})^{2} \leq a(\log a-\log b)+b-a .
$$

This inequality remains valid in the limits $a \rightarrow 0$ and $b \rightarrow 0$ as well.

(b) Let $b \geq b_{0}>0$ and $K:=1 / b_{0}$. Then

$$
a(\log a-\log b)+b-a \leq K(a-b)^{2} .
$$

Proposition 1 can be proven by elementary calculus employing the relation

$$
\frac{2}{a+b}<\frac{\log a-\log b}{a-b}<\frac{1}{\sqrt{a b}}, \quad a>b>0 .
$$

Theorem 5.1. Let $\Omega \subset \mathbb{R}^{2}$ be bounded and smooth, let $\left(u^{0}, c^{0}\right) \in W_{0, \sigma}^{1,2}(\Omega) \times$ $\left(W^{1,2}(\Omega) \cap L^{\infty}(\Omega)\right)$ with initial masses $m_{i}=\int_{\Omega} c_{i}^{0} d x>0, i=1, \ldots, N$, be given, let $(u, c)$ be the global strong solution to $(P)$ from Theorem 1.2 with initial data $\left(u^{0}, c^{0}\right)$, and let $\Psi$ be defined as in (5.1). Then there are constants $C, \omega>0$ depending on the initial data such that

$$
\Psi(u(t), c(t)) \leq C e^{-\omega t} \quad \text { for a.a. } t \geq 0 .
$$

Proof. Recall the definition of $V$ in (3.4) and note that $\frac{\mathrm{d}}{\mathrm{d} t} V=\frac{\mathrm{d}}{\mathrm{d} t} \Psi(u(\cdot), c(\cdot))$, so from (4.4) we have

$$
\frac{\mathrm{d}}{\mathrm{d} t} \Psi(u(t), c(t))=-D(u(t), c(t)),
$$

where

$$
D(u, c)=\|\nabla u(t)\|_{2}^{2}+\sum_{i=1}^{N} \int_{\Omega} \frac{D_{i}}{c_{i}(t)} \exp \left(-2 z_{i} \phi(t)\right)\left|\nabla \zeta_{i}(t)\right|^{2} d x
$$

with $\zeta_{i}=c_{i} \exp \left(z_{i} \phi\right)$. The idea of the proof is to estimate $\Psi$ suitably in terms of the dissipation rate $D$ and obtain exponential decay via Gronwall.

Let us define the auxiliary variable

$$
\psi_{i}(t):=\sqrt{\frac{c_{i}(t)}{c_{i}^{\infty}}} \exp \left(z_{i} \frac{\phi(t)-\phi^{\infty}}{2}\right)-1 .
$$


Recall that $c_{i}^{\infty} \exp \left(z_{i} \phi^{\infty}\right)=\zeta_{i}^{\infty}>0$ due to Lemma 4.3. For $c_{i} \neq 0$, the gradient of $\psi_{i}$ satisfies

$$
\begin{aligned}
\left|\nabla \psi_{i}(t)\right|^{2} & =\frac{1}{4 c_{i}(t) c_{i}^{\infty} \exp \left(z_{i}\left(\phi^{\infty}-\phi(t)\right)\right)}\left|\nabla c_{i}(t)+z_{i} c_{i}(t) \nabla \phi(t)\right|^{2} \\
& \leq \frac{C D_{i}}{c_{i}(t)} \exp \left(-z_{i} \phi(t)\right)\left|\nabla \zeta_{i}(t)\right|^{2},
\end{aligned}
$$

where we use the fact that $c_{i}^{\infty} \exp \left(z_{i} \phi^{\infty}\right)=$ const and $\|\phi\|_{\infty} \leq C$ uniformly in time, so

$$
\|\nabla u(t)\|_{2}^{2}+\sum_{i=1}^{N}\left\|\nabla \psi_{i}(t)\right\|_{2}^{2} \leq C D(u(t), c(t)) .
$$

For proving the assertion it is sufficient to estimate

$$
\Psi(u(t), c(t)) \leq C\left(\|\nabla u(t)\|_{2}^{2}+\sum_{i=1}^{N}\left\|\nabla \psi_{i}(t)\right\|_{2}^{2}\right)
$$

with some constant $C>0$ independent of $t$, since then, by (5.3) and Gronwall, we obtain exponential decay of $\Psi(u(\cdot), c(\cdot))$. Note that we only require (5.6) to hold true for those states $(u(t), c(t))$ which are attained by the solution $(u, c)$.

By Poincaré's inequality we already know that $\|u\|_{2} \leq C\|\nabla u\|_{2}$ for all $u \in W_{0, \sigma}^{1,2}(\Omega)$. So for (5.6) it suffices to show that for given $R, R^{\prime}>0$ we have

$$
\begin{aligned}
& \tilde{\Psi}(c):= \\
& :=\sum_{i=1}^{N} \int_{\Omega}\left(c_{i}\left(\log \frac{c_{i}}{c_{i}^{\infty}}-1\right)+c_{i}^{\infty}\right) d x+\frac{\varepsilon}{2}\left\|\nabla\left(\phi-\phi^{\infty}\right)\right\|_{2}^{2}+\frac{\varepsilon \tau}{2}\left\|\phi-\phi^{\infty}\right\|_{2, \partial \Omega}^{2} \\
& \leq C \sum_{i}\left\|\nabla \psi_{i}\right\|_{2}^{2}
\end{aligned}
$$

for all $c \in Z_{R, R^{\prime}}$ with

$$
\begin{aligned}
& Z_{R, R^{\prime}}:=\left\{v \in W^{1,2}(\Omega) \cap L^{\infty}(\Omega):\right. \\
&\left.\qquad v_{i} \geq 0, \int_{\Omega} v_{i}=m_{i}, \tilde{\Psi}(v) \leq R,\|v\|_{\infty} \leq R^{\prime}\right\},
\end{aligned}
$$

where $\phi \in W^{3,2}(\Omega)$ is the unique solution to

$$
-\varepsilon \Delta \phi=\sum_{i=1}^{N} z_{i} c_{i} \text { in } \Omega, \quad \partial_{\nu} \phi+\tau \phi=\xi \text { on } \partial \Omega .
$$

Note that for given initial data with initial masses $m_{i}>0$ the phase space of $c$ is contained in $Z_{R, R^{\prime}}$ for sufficiently large $R, R^{\prime}>0$, cf. Corollary 1 , 
Remark 9, and Lemma 3.13. From the Sobolev embedding $W^{2,2}(\Omega) \hookrightarrow$ $B U C(\bar{\Omega})$ we compute

$$
\|\phi\|_{\infty} \leq C\|\phi\|_{W^{2,2}} \leq C^{\prime}\|c\|_{2} \leq C^{\prime \prime}\|c\|_{\infty} \leq C^{\prime \prime} R^{\prime}
$$

for $c \in Z_{R, R^{\prime}}$, so we observe that the corresponding potentials $\phi$ are uniformly bounded in $L^{\infty}(\Omega)$.

For the proof of relation (5.7) observe that by the boundedness of $c_{i}^{\infty}$ from below due to Lemma 4.3 and Proposition 1 there are constants $C_{1}, C_{2}>0$ such that

$$
\begin{aligned}
C_{1}\left(\sum_{i}\left\|\sqrt{c_{i}}-\sqrt{c_{i}^{\infty}}\right\|_{2}^{2}+\left\|\phi-\phi^{\infty}\right\|_{W^{1,2}}^{2}\right) & \leq \tilde{\Psi}(c) \\
& \leq C_{2}\left(\sum_{i}\left\|c_{i}-c_{i}^{\infty}\right\|_{2}^{2}+\left\|\phi-\phi^{\infty}\right\|_{W^{1,2}}^{2}\right)
\end{aligned}
$$

for all $c \geq 0$ and corresponding potentials $\phi$.

We will derive (5.7) indirectly. So suppose relation (5.7) does not hold. Step 1.

Then there is a sequence $l_{k} \rightarrow \infty$ and $\left(c^{k}\right)_{k} \subset Z_{R, R^{\prime}}$ with corresponding $\phi^{k}$, solution to

$$
-\varepsilon \Delta \phi^{k}=\sum_{i} z_{i} c_{i}^{k}, \text { in } \Omega, \quad \partial_{\nu} \phi^{k}+\tau \phi^{k}=\xi, \text { on } \partial \Omega,
$$

and auxiliary functions $\psi_{i}^{k}$, given by

$$
\psi_{i}^{k}=\sqrt{\frac{c_{i}^{k}}{c_{i}^{\infty}}} \exp \left(z_{i} \frac{\phi^{k}-\phi^{\infty}}{2}\right)-1,
$$

such that

$$
l_{k} \sum_{i}\left\|\nabla \psi_{i}^{k}\right\|_{2}^{2} \leq \tilde{\Psi}\left(c^{k}\right) \leq R .
$$

Note that for $c_{i}^{k}, \psi_{i}^{k}$ superscripts refer to the index of the sequence and subscripts to the corresponding components. Without loss of generality we may exclude the case that $c^{k}=c^{\infty}$ for some $k \in \mathbb{N}$, since in this case inequality (5.7) is trivially satisfied.

By relation (5.11) we see $\nabla \psi_{i}^{k} \rightarrow 0$ in $L^{2}(\Omega)$ for $k \rightarrow \infty$. Due to the boundeds for $c^{k}$ and $\phi^{k}$, implied by the definiton of $Z_{R, R^{\prime}}$ and (5.8), we see that $\left\|\psi_{i}^{k}\right\|_{2}$ can be bounded by a constant independent of $k$, cf. (5.10). Hence the sequence $\left(\psi_{i}^{k}\right)_{k}$ is bounded in $W^{1,2}(\Omega)$, and therefore $\psi_{i}^{k} \rightarrow \hat{\psi}_{i}$ in $W^{1,2}(\Omega)$ and $\psi_{i}^{k} \rightarrow \hat{\psi}_{i}$ in $L^{2}(\Omega)$ via compactness. Because both $\psi_{i}^{k} \rightarrow \hat{\psi}_{i}$ and $\nabla \psi_{i}^{k} \rightarrow 0$ in $L^{2}(\Omega)$, it follows that

$$
\psi_{i}^{k} \rightarrow \hat{\psi}_{i} \text { in } W^{1,2}(\Omega) \text { as } k \rightarrow \infty,
$$

where $\nabla \hat{\psi}_{i}=0$, hence $\hat{\psi}_{i}=$ const. 
By (5.9) we have $\left\|\phi^{k}-\phi^{\infty}\right\|_{W^{1,2}} \leq C$, so there is $\hat{\phi} \in W^{1,2}(\Omega)$ such that

$$
\phi^{k} \rightarrow \hat{\phi} \text { in } W^{1,2}(\Omega) \text {, hence } \phi^{k} \rightarrow \hat{\phi} \text { in } L^{2}(\Omega) \text {, as } k \rightarrow \infty,
$$

again using compactness. From the uniform boundedness of $\left\|\phi^{k}\right\|_{\infty}$ by (5.8) and its convergence almost everywhere we deduce $\|\hat{\phi}\|_{\infty}<\infty$. Let

$$
\hat{c}_{i}:=c_{i}^{\infty}\left(1+\hat{\psi}_{i}\right)^{2} \exp \left(z_{i}\left(\phi^{\infty}-\hat{\phi}\right)\right) .
$$

By the definition of $\psi_{i}^{k}$ in (5.10) we have $c_{i}^{k}=c_{i}^{\infty}\left(1+\psi_{i}^{k}\right)^{2} \exp \left(z_{i}\left(\phi^{\infty}-\phi^{k}\right)\right)$. Straight forward manipulations reveal

$$
\begin{aligned}
c_{i}^{k}-\hat{c}_{i}= & c_{i}^{\infty} \exp \left(z_{i}\left(\phi^{\infty}-\phi^{k}\right)\right)\left[\left(1+\psi_{i}^{k}\right)^{2}-\left(1+\hat{\psi}_{i}\right)^{2} \exp \left(z_{i}\left(\phi^{k}-\hat{\phi}\right)\right)\right] \\
= & c_{i}^{\infty} \exp \left(z_{i}\left(\phi^{\infty}-\phi^{k}\right)\right)\left[\left(\psi_{i}^{k}-\hat{\psi}_{i}\right)^{2}+2\left(\psi_{i}^{k}-\hat{\psi}_{i}\right)+2 \hat{\psi}_{i}\left(\psi_{i}^{k}-\hat{\psi}_{i}\right)\right. \\
& \left.+\left(1+\hat{\psi}_{i}\right)^{2}\left(1-\exp \left(z_{i}\left(\phi^{k}-\hat{\phi}\right)\right)\right)\right] .
\end{aligned}
$$

Using the a priori estimates $\left\|\phi^{k}\right\|_{\infty}+\|\hat{\phi}\|_{\infty}+\left\|\phi^{\infty}\right\|_{\infty}+\left\|c^{\infty}\right\|_{\infty} \leq C$ and the fact that $W^{1,2}(\Omega) \hookrightarrow L^{4}(\Omega)$ gives

$$
\left\|c_{i}^{k}-\hat{c}_{i}\right\|_{2} \leq C\left(\left\|\psi_{i}^{k}-\hat{\psi}_{i}\right\|_{4}^{2}+\left\|\psi_{i}^{k}-\hat{\psi}_{i}\right\|_{2}+\left\|\phi^{k}-\hat{\phi}\right\|_{2}\right) \rightarrow 0 \text { as } k \rightarrow \infty \text {. }
$$

By continuity it is true that $-\varepsilon \Delta \hat{\phi}=\sum_{i} z_{i} \hat{c}_{i}$ in $\Omega$, and $\partial_{\nu} \hat{\phi}+\tau \hat{\phi}=\xi$ on $\partial \Omega$, and

$$
\hat{c}_{i} \exp \left(z_{i} \hat{\phi}\right)=c_{i}^{\infty} \exp \left(z_{i} \phi^{\infty}\right)\left(1+\hat{\psi}_{i}\right)^{2} \equiv \text { const. }
$$

From relation (5.14) we readily approve that $\hat{c}_{i} \in W^{1,2}(\Omega)$, since both $\hat{\psi}_{i}$ and $c_{i}^{\infty} \exp \left(z_{i} \phi^{\infty}\right)$ are constant and $\hat{\phi} \in W^{1,2}(\Omega)$. Thus $(\hat{c}, \hat{\phi})$ is the unique steady state to $(P)$ from Lemma 4.3 and Theorem 1.3, i.e. $(\hat{c}, \hat{\phi})=\left(c^{\infty}, \phi^{\infty}\right)$. So relation (5.15) shows

$$
c_{i}^{k} \rightarrow c_{i}^{\infty} \text { in } L^{2}(\Omega) \text { as } k \rightarrow \infty .
$$

For the sequence $\left(\tilde{\Psi}\left(c^{k}\right)\right)_{k}$ this implies

$$
\lambda_{k}^{2}:=\tilde{\Psi}\left(c_{k}\right) \rightarrow 0
$$

by (5.9).

Step 2.

Recall that we assume $c_{k} \neq c^{\infty}$ for $k \in \mathbb{N}$, so $\lambda_{k}^{2}=\tilde{\Psi}\left(c_{k}\right)>0$ for all $k \in \mathbb{N}$. Having obtained the convergence of the sequences $\left(\psi_{i}^{k}\right)_{k},\left(\phi^{k}\right)_{k},\left(c_{i}^{k}\right)_{k}$ and $\lambda_{k}$ in (5.12), (5.13), (5.16) and (5.17) we define new variables

$$
w_{i}^{k}:=\frac{\psi_{i}^{k}}{\lambda_{k}}, \quad y_{i}^{k}:=\frac{c_{i}^{k}-c^{\infty}}{\lambda_{k}}, \quad \chi^{k}:=\frac{\phi^{k}-\phi^{\infty}}{\lambda_{k}} .
$$

Again for $w_{i}^{k}$ and $y_{i}^{k}$ superscripts represent the index of the sequence and subscripts denote the respective components. Note that for $k \in \mathbb{N}$ the function $\chi^{k}$ is the unique solution to

$$
-\varepsilon \Delta \chi^{k}=\sum_{i} z_{i} y_{i}^{k} \quad \text { in } \Omega, \text { and } \partial_{\nu} \chi^{k}+\tau \chi^{k}=0 \quad \text { on } \partial \Omega \text {. }
$$


Dividing (5.11) by $l_{k} \lambda_{k}^{2}$ it follows that $\sum_{i}\left\|\nabla w_{i}^{k}\right\|_{2}^{2} \leq 1 / l_{k}$, so $\nabla w_{i}^{k} \rightarrow 0$ in $L^{2}(\Omega)$. We shall argue in an analogue way as for (5.12). For the $L^{2}$ boundedness of $w_{i}^{k}$, observe that $\psi_{i}^{k}$ can be rewritten as

$$
\psi_{i}^{k}=\frac{1}{\sqrt{c_{i}^{\infty}}}\left(\sqrt{c_{i}^{k}}-\sqrt{c_{i}^{\infty}}\right)+\sqrt{\frac{c_{i}^{k}}{c_{i}^{\infty}}}\left(\exp \left(z_{i} \frac{\phi^{k}-\phi^{\infty}}{2}\right)-1\right) .
$$

With the help of (5.9) we conclude

$$
\begin{aligned}
\sum_{i=1}^{N}\left\|\psi_{i}^{k}\right\|_{2}^{2}+\left\|\phi^{k}-\phi^{\infty}\right\|_{W^{1,2}}^{2} & \leq C\left(\sum_{i=1}^{N}\left\|\sqrt{c_{i}^{k}}-\sqrt{c_{i}^{\infty}}\right\|_{2}^{2}+\left\|\phi^{k}-\phi^{\infty}\right\|_{W^{1,2}}^{2}\right) \\
& \leq C^{\prime} \tilde{\Psi}\left(c^{k}\right) .
\end{aligned}
$$

Dividing (5.20) by $\lambda_{k}^{2}=\tilde{\Psi}\left(c_{k}\right)$ we have $\left\|w_{i}^{k}\right\|_{2} \leq C^{\prime}$ independent of $k$, hence

$$
w_{i}^{k} \rightarrow \hat{w}_{i} \text { in } W^{1,2}(\Omega) \text { as } k \rightarrow \infty,
$$

where $\hat{w}$ is constant. Also from the division of (5.20) by $\lambda_{k}^{2}$ we infer in the same way as for (5.13) the existence of a $\hat{\chi} \in W^{1,2}(\Omega)$ such that

$$
\chi^{k} \rightarrow \hat{\chi} \text { in } W^{1,2}(\Omega) \text {, hence } \chi^{k} \rightarrow \hat{\chi} \text { in } L^{2}(\Omega) \text {, as } k \rightarrow \infty \text {. }
$$

With relations (5.21) and (5.22) we define

$$
\hat{y}_{i}:=c_{i}^{\infty}\left(2 \hat{w}_{i}-z_{i} \hat{\chi}\right) .
$$

For the difference $y_{i}^{k}-\hat{y}_{i}$ we obtain

$$
\begin{aligned}
y_{i}^{k}-\hat{y}_{i}=c_{i}^{\infty}\left(\frac{1}{\lambda_{k}}\left(\left(1+\psi_{i}^{k}\right)^{2} \exp \left(z_{i}\left(\phi^{\infty}-\phi^{k}\right)\right)-1\right)-2 \hat{w}_{i}+z_{i} \hat{\chi}\right) \\
=c_{i}^{\infty}\left(2 \frac{\psi_{i}^{k}}{\lambda_{k}}\left(\exp \left(z_{i}\left(\phi^{\infty}-\phi^{k}\right)\right)-1\right)+2\left(\frac{\psi_{i}^{k}}{\lambda_{k}}-\hat{w}_{i}\right)+z_{i}\left(\hat{\chi}-\frac{\phi^{k}-\phi^{\infty}}{\lambda_{k}}\right)\right. \\
\left.+\frac{\left(\psi_{i}^{k}\right)^{2}}{\lambda_{k}^{2}} \lambda_{k} \exp \left(z_{i}\left(\phi^{\infty}-\phi^{k}\right)\right)+\frac{\exp \left(z_{i}\left(\phi^{\infty}-\phi^{k}\right)\right)-1-z_{i}\left(\phi^{\infty}-\phi^{k}\right)}{\lambda_{k}}\right) \\
=c_{i}^{\infty}\left(2 w_{i}^{k}\left(\exp \left(z_{i}\left(\phi^{\infty}-\phi^{k}\right)\right)-1\right)+2\left(w_{i}^{k}-\hat{w}_{i}\right)+z_{i}\left(\hat{\chi}-\chi^{k}\right)\right. \\
\left.+\lambda_{k}\left(w_{i}^{k}\right)^{2} \exp \left(z_{i}\left(\phi^{\infty}-\phi^{k}\right)\right)+\frac{\lambda_{k}}{2} z_{i}^{2}\left(\chi^{k}\right)^{2} \exp \left(h z_{i}\left(\phi^{\infty}-\phi^{k}\right)\right)\right)
\end{aligned}
$$

for some function $h \in[0,1]$ using Taylor's formula. Hence

$$
\left\|y_{i}^{k}-\hat{y}_{i}\right\|_{2} \leq C\left(\left\|\phi^{k}-\phi^{\infty}\right\|_{4}^{2}+\left\|w_{i}^{k}-\hat{w}_{i}\right\|_{2}+\lambda_{k}+\left\|\chi^{k}-\hat{z}\right\|_{2}\right) \rightarrow 0 .
$$

By continuity it follows from (5.19) that

$$
-\varepsilon \Delta \hat{\chi}=\sum_{i} z_{i} \hat{y}_{i} \text { in } \Omega, \text { and } \partial_{\nu} \hat{\chi}+\tau \hat{\chi}=0 \text { on } \partial \Omega,
$$


and that $\chi^{k} \rightarrow \hat{\chi}$ in $W^{2,2}(\Omega)$. Therefore, using integration by parts and representation (5.23), we obtain

$$
\begin{aligned}
0 & \leq \varepsilon\|\nabla \hat{\chi}\|_{2}^{2}+\varepsilon \tau\|\hat{\chi}\|_{2, \partial \Omega}^{2}+\int_{\Omega} \sum_{i} c_{i}^{\infty}\left(2 \hat{w}_{i}-z_{i} \hat{\chi}\right)^{2} \\
& =-\varepsilon \int_{\Omega} \hat{\chi} \Delta \hat{\chi}+\int_{\Omega} \sum_{i} c_{i}^{\infty}\left(2 \hat{w}_{i}-z_{i} \hat{\chi}\right)^{2} \\
& =\sum_{i} \int_{\Omega} z_{i} \hat{\chi} \hat{y}_{i}+\hat{y}_{i}\left(2 \hat{w}_{i}-z_{i} \hat{\chi}\right)=2 \sum_{i} \int_{\Omega} \hat{y}_{i} \hat{w}_{i}=0,
\end{aligned}
$$

since $\int_{\Omega} y_{i}^{k}=0$ for all $k$. Therefore $\hat{\chi}=0, \hat{w}=0$ and finally $\hat{y}_{i}=0$. However, dividing (5.9) by $\lambda_{k}^{2}$ now implies that

$$
1 \leq \sum_{i}\left\|y_{i}^{k}\right\|_{2}^{2}+\left\|\chi^{k}\right\|_{W^{1,2}}^{2} \rightarrow 0 \text { as } k \rightarrow \infty
$$

a contradiction.

Now the proof of Theorem 1.4 remains an easy task.

Proof. (of Theorem 1.4). From the definition of $\Psi$ in (5.1) it is clear that $\Psi \geq 0$, so Theorem 5.1 implies $\|u(t)\|_{2} \leq C e^{-\omega t}$. The claim for $c$ follows with the help of the estimates in Lemma 3.13. Theorem 5.1 and relation (5.9) by

$$
\left\|c_{i}-c_{i}^{\infty}\right\|_{1} \leq C\left\|\left(\sqrt{c_{i}}+\sqrt{c_{i}^{\infty}}\right)\left(\sqrt{c_{i}}-\sqrt{c_{i}^{\infty}}\right)\right\|_{2} \leq C^{\prime} \Psi(u, c)^{1 / 2} \leq C^{\prime \prime} e^{-\omega^{\prime} t} .
$$

\section{ACKNOWLEDGEMENT}

This work is supported by the Deutsche Forschungsgemeinschaft within the cluster of excellence "Center of Smart Interfaces" at the TU Darmstadt. The authors would like to thank A. Glitzky for useful hints concerning the boundary conditions (1.21) imposed on the electro-static potential.

\section{REFERENCES}

[1] R.A. Adams and J.J.F. Fournier. Sobolev Spaces. Academic press, Kidlington, Oxford, 2 edition, 2003.

[2] H. Amann. Linear and Quasilinear Parabolic Problems - Abstract Linear Theory, volume 1 of Monographs in mathematics. Birkhäuser Verlag Basel, 1994.

[3] H. Amann and M. Renardy. Reaction-diffusion problems in electrolysis. Nonlinear Differential Equations and Applications, pages 91-117, 1994.

[4] P.W. Atkins and J. de Paula. Physikalische Chemie. Wiley, 4 edition, 2006.

[5] L. Bedin and M. Thompson. Existence theory for a Poisson-Nernst-Planck model of electrophoresis. arxiv:1102.5370v1, 2011.

[6] R.B. Bird, W.E. Stewart, and E.N. Lightfoot. Transport Phenomena. Wiley, 2 edition, 2001. 
[7] D. Bothe and J. Prüss. Mass transport through charged membranes. Proc. 4th European Conf. on Elliptic and Parabolic Problems, pages 332-342, 2002.

[8] S.T. Chang. New Electrokinetic Techniques for Material Manipulation on the Microscale. BiblioBazaar, 2011.

[9] Y.S. Choi and R. Lui. Analysis of an electrochemistry model with zero-flux boundary conditions. Appl. Anal., 49:277-288, 1993.

[10] Y.S. Choi and R. Lui. Multi-dimensional electrochemistry model. Arch. Rational Mech. Anal., 130:315-342, 1995.

[11] E.L. Cussler. Diffusion: Mass Transfer in Fluid Systems. Cambridge University Press, 2 edition, 1997.

[12] R. Denk, M. Hieber, and J. Prüss. Optimal $L^{p}-L^{q}$-regularity for parabolic problems with inhomogeneous boundary data. Math. Z., 257:193-224, 2007.

[13] L. Desvillettes and K. Fellner. Exponetial decay toward equilibrium via entropy methods for reaction-diffusion equations. Journal of Mathematical Analysis and Applications, 319:157-176, 2006.

[14] L.C. Evans. Partial differential equations. Providence, RI: American Mathematical Society, 1998.

[15] H. Gajewski. On existence, uniqueness and asymptotic behaviour of solutions of the basic equations for carrier transport in semiconductors. Z. Angew. Math. u. Mech, 65:101-108, 1985.

[16] G.P. Galdi. An Introduction to the Mathematical Theory of the Navier-Stokes Equations, Vol. I, Linearised Steady Problems. Springer Verlag, New York, 2 edition, 2011.

[17] Y. Giga. Domains of fractional powers of the Stokes operator in $L_{r}$ spaces. Arch. Ration. Mech. Anal., 89:251-265, 1985.

[18] D. Gilbarg and N.S. Trudinger. Elliptic Partial Differential Equations of Second Order. Springer, 2001.

[19] A. Glitzky, K. Gröger, and R. Hünlich. Free energy and dissipation rate for reaction diffusion processes of electrically charged species. Appl. Anal., 60:201-217, 1995.

[20] A. Glitzky and R. Hünlich. Energetic estimates and asymptotics for electro-reactiondiffusion systems. Z. Angew. Math. Mech., 77:823-832, 1997.

[21] A. Glitzky and R. Hünlich. Global estimates and asymptotics for electro-reactiondiffusion systems in heterostructures. Appl. Anal., 66:205-226, 1997.

[22] C.H. Hamann, A. Hamnett, and W. Wielstich. Electrochemistry. Wiley-VCH, 1998.

[23] J.W. Jerome. Analytical approaches to charge transport in a moving medium. Transport Theory and Statistical Physics, 31(4-6):333-336, 2002.

[24] B. Kirby. Micro- and Nanoscale Fluid Mechanics: Transport in Microfluidic Devices. Cambridge University Press, 2010.

[25] M. Köhne, J. Prüss, and M. Wilke. Qualitative behaviour of solutions for the twophase Navier-Stokes equations with surface tension. Math. Ann. to appear.

[26] R. Krishna and R. Taylor. Multicomponent mass transfer. Wiley (New York), 1993.

[27] O.A. Ladyzenskaja, V.A. Solonnikov, and N.N. Ural'seca. Linear and quasilinear equations of parabolic type, volume 23 of Translation of Mathematical Monographs. Amer. Math. Soc, 1968.

[28] J.H. Masliyah and S. Bhattacharjee. Electrokinetic and Colloid Transport Phenomena. Wiley-Interscience, 1993.

[29] M. Meyries and R. Schnaubelt. Interpolation, embeddings and traces of anisotropic fractional Sobolev spaces with temporal weights. J. Funct. Anal., 262:1200-1229, 2012.

[30] S. Movahed and D. Li. Electrokinetic transport through nanochannels. Electrophoresis, 32:1259-1267, 2011.

[31] J.S. Newman. Electrochemical systems. Prentice Hall, 2 edition, 1991. 
[32] A. Noll and J. Saal. $H^{\infty}$-calculus for the Stokes operator on $L_{q}$-spaces. Math.Z., 244:651-688, 2003.

[33] R.F. Probstein. Physicochemical Hydrodynamics. Butterworths, 1989.

[34] J. Prüss, J. Saal, and G. Simonett. Existence of analytic solutions for the classical Stefan problem. Math. Ann., 338:703-755, 2007.

[35] M. Schmuck. Analysis of the Navier-Stokes-Nernst-Planck-Poisson system. Mathematical Models and Methods in Applied Sciences, 19:993-1014, 2009.

[36] P.E. Sobolevskii. Fractional powers of coercively positive sums of operators. Soviet Math. Dokl., 6:1638-1641, 1975.

[37] H. Sohr. The Navier-Stokes Equations: An Elementary Functional Analytic Approach. Birkhäuser, Boston Basel Berlin, 2001.

[38] V.A. Solonnikov. Estimates for solutions of nonstationary Navier-Stokes equations. J. Soviet Math, 8:213-317, 1977.

[39] R. Temam. Infinite-Dimensional Dynamical Systems in Mechanics and Physics, volume 68 of Applied Mathematical Sciences. Springer New York, 2 edition, 1997.

[40] H. Triebel. Interpolation Theory, Function Spaces, Differential Operators. North Holland, 1978.

[41] M. Trojanowicz. Advances in Flow Analysis. Wiley-VCH, 2008.

[42] J. Wiedmann. An electrolysis model and its solutions. Phd-thesis, University of Zurich, 1997.

[43] Z. Wu, J. Yin, and C. Wang. Elliptic and Parabolic Equations. World Scientific Publishing, 2006.

Center of Smart Interfaces, Technische Universität Darmstadt, PetersenStrasse 32, 64287 Darmstadt, Germany

E-mail address: bothe@csi.tu-darmstadt.de

Center of Smart Interfaces, Technische Universität Darmstadt, PetersenStrasse 32, 64287 Darmstadt, Germany

E-mail address: fischer@csi.tu-darmstadt.de

Center of Smart Interfaces, Technische Universität Darmstadt, Petersenstrasse 32, 64287 Darmstadt, Germany

E-mail address: saal@csi.tu-darmstadt.de 